\title{
A Triangular Palladium Cluster from the Activation of the Si-Si bond in a Disilane with Phosphine Pendants
}

\author{
Ryosuke Usuił and Yusuke Sunada ${ }^{*,+, t, \xi}$
}

Department of Applied Chemistry, School of Engineering Science, and †Institute of Industrial

Science, The University of Tokyo, 4-6-1 Komaba, Meguro-ku, Tokyo 153-8505, Japan.

§JST PRESTO, 4-1-8 Honcho, Kawaguchi, Saitama, 332-0012 Japan

\section{Contents}

1. General

p. S-2

2. Synthesis of $\mathbf{2}$ and $\mathbf{3}$

p. S-2

3. Suzuki-Miyaura coupling catalyzed by 3

p. S-3

4. Monitoring the reaction of $\left[\mathrm{Pd}\left(\mathrm{CN}^{t} \mathrm{Bu}\right)_{2}\right]_{3}$ with disilane 2

p. S-3

5. Reaction of $\left[\mathrm{Pd}\left(\mathrm{CN}{ }^{t} \mathrm{Bu}\right)_{2}\right]_{3}$ with $\mathrm{PPh}_{3}$

p. S-3

6. ${ }^{1} \mathrm{H},{ }^{13} \mathrm{C},{ }^{29} \mathrm{Si}$ and ${ }^{31} \mathrm{P} \mathrm{NMR}$ spectra of 2

p. S-4

7. ${ }^{1} \mathrm{H},{ }^{13} \mathrm{C},{ }^{29} \mathrm{Si}$ and ${ }^{31} \mathrm{P}$ NMR spectra and IR spectrum of 3

p. S-6

8. Actual NMR chart of the crude product obtained from the reaction of $\left[\mathrm{Pd}\left(\mathrm{CN}^{t} \mathrm{Bu}\right)_{2}\right]_{3}$ with disilane 2

9. Actual NMR chart of the crude product obtained from the reaction of $\left[\mathrm{Pd}\left(\mathrm{CN} \mathrm{Bu}_{2}\right]_{3}\right.$ with $\mathrm{PPh}_{3}$

6. Theoretical calculation

p. S-12

7. X-ray diffraction analysis

p. $\mathrm{S}-20$

8. References

p. S-47 
General. Manipulation of air and moisture sensitive compounds was carried out under a dry nitrogen atmosphere using Schlenk tube techniques associated with a high-vacuum line or in the glove box which was filled with dry nitrogen. All solvents were purchased from Kanto Chemical Co. Inc., and was dried over activated molecular sieves. ${ }^{1} \mathrm{H},{ }^{13} \mathrm{C},{ }^{29} \mathrm{Si}$ NMR spectra were recorded on a JEOL Lambda 400 spectrometer at ambient temperature unless otherwise noted. ${ }^{1} \mathrm{H},{ }^{13} \mathrm{C},{ }^{29} \mathrm{Si}$ NMR chemical shifts ( $\delta$ values) were given in ppm relative to the solvent signal $\left({ }^{1} \mathrm{H},{ }^{13} \mathrm{C}\right)$ or standard resonances $\left({ }^{29} \mathrm{Si}\right.$ : external tetramethylsilane). Elemental analyses were performed by a Thermo Scientific FLASH 2000 Organic Elemental Analyzer. IR spectra were recorded on a PerkinElmer Spectrum Two spectrometer. The starting compounds, $\mathrm{Pd}\left(\mathrm{CN}{ }^{t} \mathrm{Bu}\right)_{2}{ }^{1}$ and $\mathrm{ClPh}_{2} \mathrm{Si}_{-}$ $\mathrm{SiPh}_{2} \mathrm{Cl}^{2}$ were synthesized by the method reported in the literature. All reagents were purchased from Tokyo Chemical Industries Co., Ltd. or Sigma-Aldrich, and were used without further purification.

Synthesis of ( $\left.\mathbf{P h}_{2} \mathbf{P C H}_{2}\right) \mathbf{P h} \mathbf{S h}_{\mathbf{2}} \mathbf{S i}-\mathbf{S i P h}_{\mathbf{2}}\left(\mathbf{C H}_{\mathbf{2}} \mathbf{P P h}\right)$ (2). In a $50 \mathrm{~mL}$ schlenk tube, $\mathrm{ClPh}_{2} \mathrm{Si}_{2} \mathrm{SiPh}_{2} \mathrm{Cl}$ (490 mg, 1.13 mmol) was dissolved in THF $(10 \mathrm{~mL})$, then THF solution of (TMEDA)Li( $\left.\mathrm{CH}_{2} \mathrm{PPh}_{2}\right)$ (2.26 mmol) (prepared by the reaction of methyldiphenylphosphine with 1 equiv. of ${ }^{n} \mathrm{BuLi}$ in the presence of 1 equiv. of TMEDA in THF) was added to this solution at $-78{ }^{\circ} \mathrm{C}$. The solution was stirred at room temperature for overnight. The solvent was removed in vacuo, and the obtained solid was washed with $\mathrm{H}_{2} \mathrm{O}(3 \times 10 \mathrm{~mL})$ and diethyl ether (3 $\mathrm{x} 10 \mathrm{~mL} \mathrm{~mL}$ ). The resulting solid was dried in vacuo to afford 2 as colorless crystals in $56 \%$ yield (483 $\mathrm{mg}$, $0.63 \mathrm{mmol}$ ). ${ }^{1} \mathrm{H}$ NMR (400 MHz, r.t., $\left.\mathrm{C}_{6} \mathrm{D}_{6}\right): \delta=2.33$ (d, 4H, $\left.-\mathrm{CH}_{2^{-}}\right), 6.94-7.08$ (m, $24 \mathrm{H}, \mathrm{Ph}$ ), 7.31-7.36 (m, 8H, Ph), 7.56-7.59 (m, 4H, Ph). ${ }^{13} \mathrm{C}$ NMR (100 MHz, r.t., $\left.\mathrm{C}_{6} \mathrm{D}_{6}\right): 12.71$ (d, J=35 Hz, $-\mathrm{CH}_{2}$ ), 127.95 (s, Ph), 129.32 (s, Ph), 133.00 (s, Ph), 133.16 (s, Ph), 135.42 (s, Ph), 136.76 (s, Ph), 141.28 (s, Ph), 141.44 (s, Ph). ${ }^{29} \mathrm{Si}$ NMR (119 MHz, r.t., $\left.\mathrm{C}_{6} \mathrm{D}_{6}\right)$ : $-20.8(\mathrm{~d}, J=19.4 \mathrm{~Hz}) .{ }^{31} \mathrm{P}$ NMR $\left(162 \mathrm{MHz}\right.$, r.t., $\left.\mathrm{C}_{6} \mathrm{D}_{6}\right):-22.2$ (s, with a satellite signal due to ${ }^{29} \mathrm{Si}, J=19.4 \mathrm{~Hz}$ ). Anal calcd for $\mathrm{C}_{50} \mathrm{H}_{44} \mathrm{P}_{2} \mathrm{Si}_{2}$; C 78.71, H 5.81; found: C 78.61, H 6.13.

Synthesis of $\mathbf{P d}_{\mathbf{3}}$ cluster 3. In a $50 \mathrm{~mL}$ schlenk tube, disilane $\mathbf{2}(140 \mathrm{mg}, 0.18 \mathrm{mmol}$ ) was dissolved in toluene $(5 \mathrm{~mL})$, then toluene $(5 \mathrm{~mL})$ solution of $\left[\mathrm{Pd}\left(\mathrm{CN}^{t} \mathrm{Bu}\right)_{2}\right]_{3}\left(100 \mathrm{mg}, 0.12 \mathrm{mmol}, 0.36 \mathrm{mmol}\right.$ as " $\left.\mathrm{Pd}\left(\mathrm{CN}^{t} \mathrm{Bu}\right)_{2}{ }^{\prime}\right)$ was added to this solution at room temperature. The solution was stirred at $80{ }^{\circ} \mathrm{C}$ for overnight. The solvent was removed in vacuo, and the obtained solid was washed with pentane $(3 \times 2 \mathrm{~mL})$ and diethyl ether $(3 \times 2 \mathrm{~mL})$. The obtained solid was dissolved in toluene $(4 \mathrm{~mL})$ and the solution was cooled to $-20^{\circ} \mathrm{C}$, then $\mathbf{3}$ was isolated as dark red crystals in $81 \%$ yield (based on $\mathrm{Si})(171 \mathrm{mg}, 0.10 \mathrm{mmol}) .{ }^{1} \mathrm{H}$ NMR $\left(400 \mathrm{MHz}\right.$, r.t., $\left.\mathrm{C}_{6} \mathrm{D}_{6}\right): \delta=0.91$ (s, 18H, $\mathrm{CNCMe}_{3}$ ), 1.05-1.12 (triplet, $J=16.5 \mathrm{~Hz}, 4 \mathrm{H},-\mathrm{CH}_{2}$ ), 1.28 (d, $J=13.3 \mathrm{~Hz}, 2 \mathrm{H},-\mathrm{CH}_{2^{-}}$), 1.75 (doublet of triplets, $\left.J=4.6,14.2 \mathrm{~Hz}, 2 \mathrm{H},-\mathrm{CH}_{2^{-}}\right), 6.59-6.62(\mathrm{~m}, 4 \mathrm{H}, \mathrm{Ph}), 6.80-6.83(\mathrm{~m}, 2 \mathrm{H}, \mathrm{Ph}), 6.96-7.23(\mathrm{~m}, 43 \mathrm{H}, \mathrm{Ph})$, 7.44-7.46 (d, 4H, Ph), 7.58-7.60 (m, 4H, Ph), 7.64-7.65 (br, 4H, Ph), 7.75-7.77 (d, 4H, Ph). ${ }^{13} \mathrm{C}$ NMR (100 $\mathrm{MHz}$, r.t., $\left.\mathrm{C}_{6} \mathrm{D}_{6}\right): 7.50$ (br s, $\left.-\mathrm{CH}_{2}-\right), 30.10\left(\mathrm{~s}, \mathrm{CNC}\left(\mathrm{CH}_{3}\right)_{3}\right), 56.61\left(\mathrm{~s}, \mathrm{CNC}\left(\mathrm{CH}_{3}\right)_{3}\right), 126.60$ (s, Ph), 126.84 (s, Ph), 126.93 (s, Ph), 126.97 (s, Ph), 127.19 (s, Ph), 127.22 (s, Ph), 127.27 (s, Ph), 127.32 (s, Ph), 127.90 (s, Ph), 127.95 (s, Ph), 128.14 (s, Ph), 128.21 (s, Ph), 128.58 (s, Ph), 128.61 (s, Ph), 128.88 (s, Ph), 129.14 (s, Ph), 133.9 (t, $J=7.7 \mathrm{~Hz}, \mathrm{Ph}), 134.80$ (d, $J=15.4 \mathrm{~Hz}, \mathrm{Ph}), 135.05$ (d, $J=12.3 \mathrm{~Hz}, \mathrm{Ph}), 135.81$ (s, Ph), 136.34 (s, $\mathrm{Ph}), 138.89$ (s, Ph), $141.04(\mathrm{~s}, \mathrm{Ph}), 149.06$ (d, $J=24.6 \mathrm{~Hz}, \mathrm{Ph}$ ) (one peak assignable to the $\underline{C} \mathrm{~N}^{t} \mathrm{Bu}$ moiety was not detected). ${ }^{29} \mathrm{Si} \mathrm{NMR}\left(119 \mathrm{MHz}\right.$, r.t., $\left.\mathrm{C}_{6} \mathrm{D}_{6}\right):-4.24\left(\mathrm{~s},-\mathrm{CH}_{2}-\mathrm{SiPh}_{2}-\mathrm{CH}_{2^{-}}\right), 17.21$ (d, $\mathrm{Pd}_{-} \mathrm{SiPh}_{2}-{ }^{2} \mathrm{~J}_{\mathrm{P}-\mathrm{Si}}=54.6$ Hz). ${ }^{31} \mathrm{P}$ NMR (162 MHz, r.t., $\left.\mathrm{C}_{6} \mathrm{D}_{6}\right): 45.36\left(\mathrm{t}, \mathrm{PPh}_{3}, J=17.3 \mathrm{~Hz}\right.$, intensity of this signal relative to the signal 
at $142.06 \mathrm{ppm}$ was found to be 0.47$), 142.06(\mathrm{~d}, \mathrm{PPh}, J=17.3 \mathrm{~Hz})$. IR (ATR): $v_{\mathrm{CN}}=2142 \mathrm{~cm}^{-1}$. Anal calcd for $\mathrm{C}_{92} \mathrm{H}_{93} \mathrm{~N}_{2} \mathrm{P}_{3} \mathrm{Pd}_{3} \mathrm{Si}_{4} ; \mathrm{C} 63.17, \mathrm{H}$ 5.24, N 1.60; found: C 63.12, H 5.19, N 1.75.

Suzuki-Miyaura coupling catalyzed by 3 . In a $20 \mathrm{~mL}$ vial, bromobenzene ( $46 \mu \mathrm{L}, 0.44 \mathrm{mmol}$ ) [or 4bromoacetophenone ( $87 \mathrm{mg}, 0.44 \mathrm{mmol})$ ] and 4-methoxyphenyl boronic acid (67 $\mathrm{mg}, 0.44 \mathrm{mmol})$ was dissolved in THF (2.2 mL), then cluster $3\left(5 \mathrm{mg}, 2.9 \times 10^{-2} \mathrm{mmol}, 2 \mathrm{~mol} \%\right.$ based on $\left.\mathrm{Pd}\right)$ and $\mathrm{K}_{2} \mathrm{CO}_{3}(121 \mathrm{mg}$, $0.88 \mathrm{mmol}$ ) were added to this solution at room temperature. The solution was stirred at room temperature for $15 \mathrm{hrs}$. Then internal standard, anisole $(54 \mu \mathrm{L}, 0.5 \mathrm{mmol})$, was added, and the conversion of the substrates (> $99 \%$ ) and quantitative formation of the corresponding coupling products were confirmed by ${ }^{1} \mathrm{H}$ NMR analysis.

Monitoring the reaction of $\left[\mathrm{Pd}\left(\mathrm{CN}^{t} \mathrm{Bu}\right)_{2}\right]_{3}$ with disilane 2. In a J-young NMR tube, disilane 2 (10 $\mathrm{mg}, 0.013 \mathrm{mmol})$ was dissolved in $\mathrm{C}_{6} \mathrm{D}_{6}(0.3 \mathrm{~mL})$, then $\mathrm{C}_{6} \mathrm{D}_{6}(0.3 \mathrm{~mL})$ solution of $\left[\mathrm{Pd}\left(\mathrm{CN}^{t} \mathrm{Bu}\right)_{2}\right]_{3}(7 \mathrm{mg}, 8.6 \mathrm{x}$ $10^{-3} \mathrm{mmol}, 0.026 \mathrm{mmol}$ as " $\mathrm{Pd}\left(\mathrm{CN}^{t} \mathrm{Bu}\right)_{2}$ ") was added to this solution at room temperature. The solution was stirred at $80{ }^{\circ} \mathrm{C}$ for overnight, then the solvent was removed in vacuo. The remaining crude product was dissolved in $\mathrm{C}_{6} \mathrm{D}_{6}$, then ${ }^{1} \mathrm{H}$ and ${ }^{31} \mathrm{P}$ NMR spectra were collected. The actual NMR charts of the crude product were shown in Figure S4-1 and S4-2.

Reaction of $\left[\mathbf{P d}\left(\mathbf{C N}{ }^{t} \mathbf{B u}\right)_{2}\right]_{3}$ with $\mathbf{P P h}_{3}$. In a J-young NMR tube, $\mathrm{PPh}_{3}(6.7 \mathrm{mg}, 0.026 \mathrm{mmol})$ was dissolved in $\mathrm{C}_{6} \mathrm{D}_{6}(0.3 \mathrm{~mL})$, then $\mathrm{C}_{6} \mathrm{D}_{6}(0.3 \mathrm{~mL})$ solution of $\left[\mathrm{Pd}\left(\mathrm{CN}^{t} \mathrm{Bu}\right)_{2}\right]_{3}\left(7 \mathrm{mg}, 8.6 \times 10^{-3} \mathrm{mmol}, 0.026 \mathrm{mmol}\right.$ as " $\mathrm{Pd}\left(\mathrm{CN}^{t} \mathrm{Bu}\right)_{2}$ ") was added to this solution at room temperature. The solution was stirred at $80{ }^{\circ} \mathrm{C}$ for overnight, then ${ }^{31} \mathrm{P}$ NMR spectra were collected. The actual ${ }^{31} \mathrm{P}$ NMR chart of the crude product was shown in Figure S5. 
Figure S1-1. ${ }^{1} \mathrm{H}$ NMR spectrum of solution of 2 in $\mathrm{C}_{6} \mathrm{D}_{6}$ at room temperature.

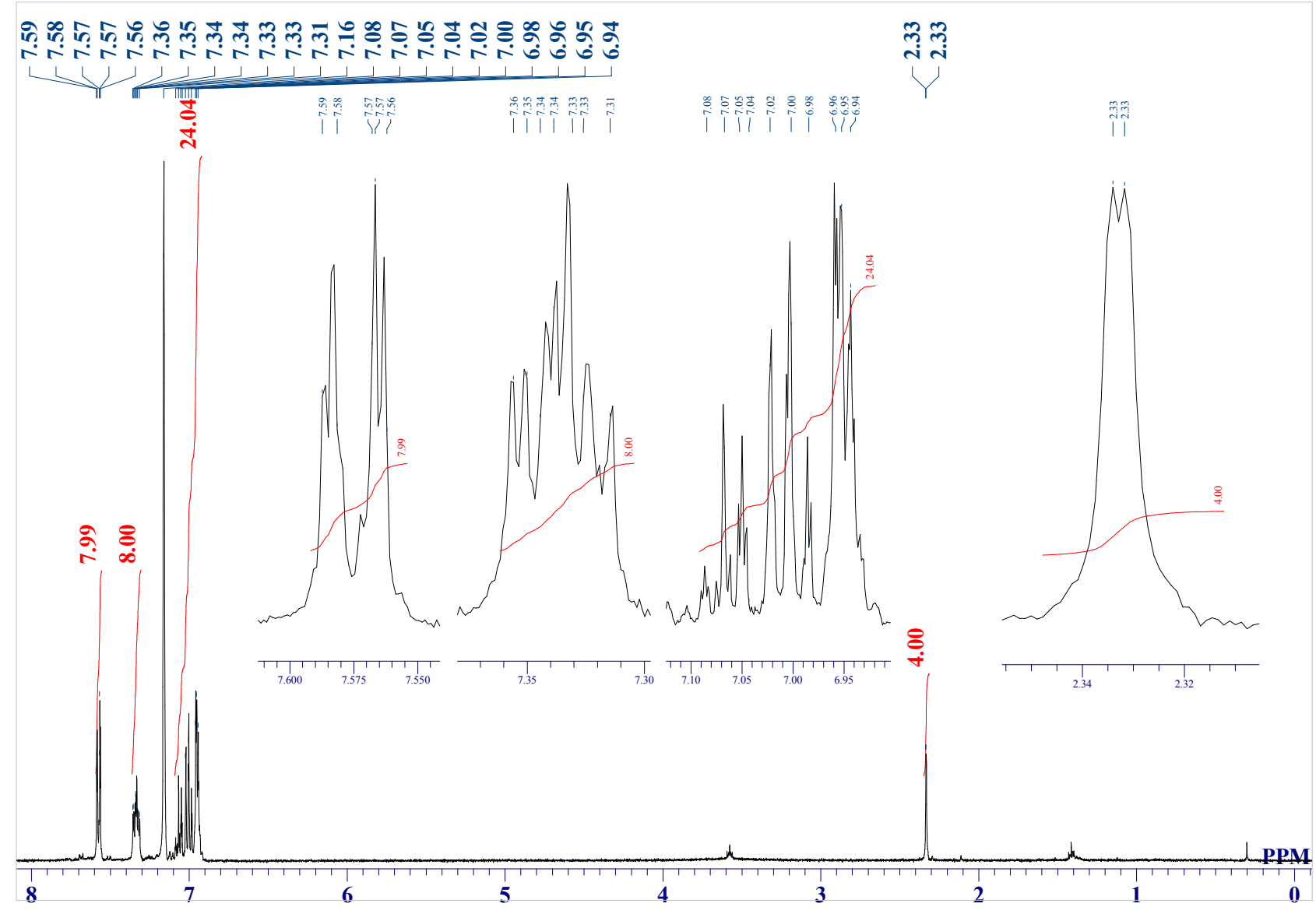

Figure S1-2. ${ }^{13} \mathrm{C}$ NMR spectrum of solution of 2 in $\mathrm{C}_{6} \mathrm{D}_{6}$ at room temperature.

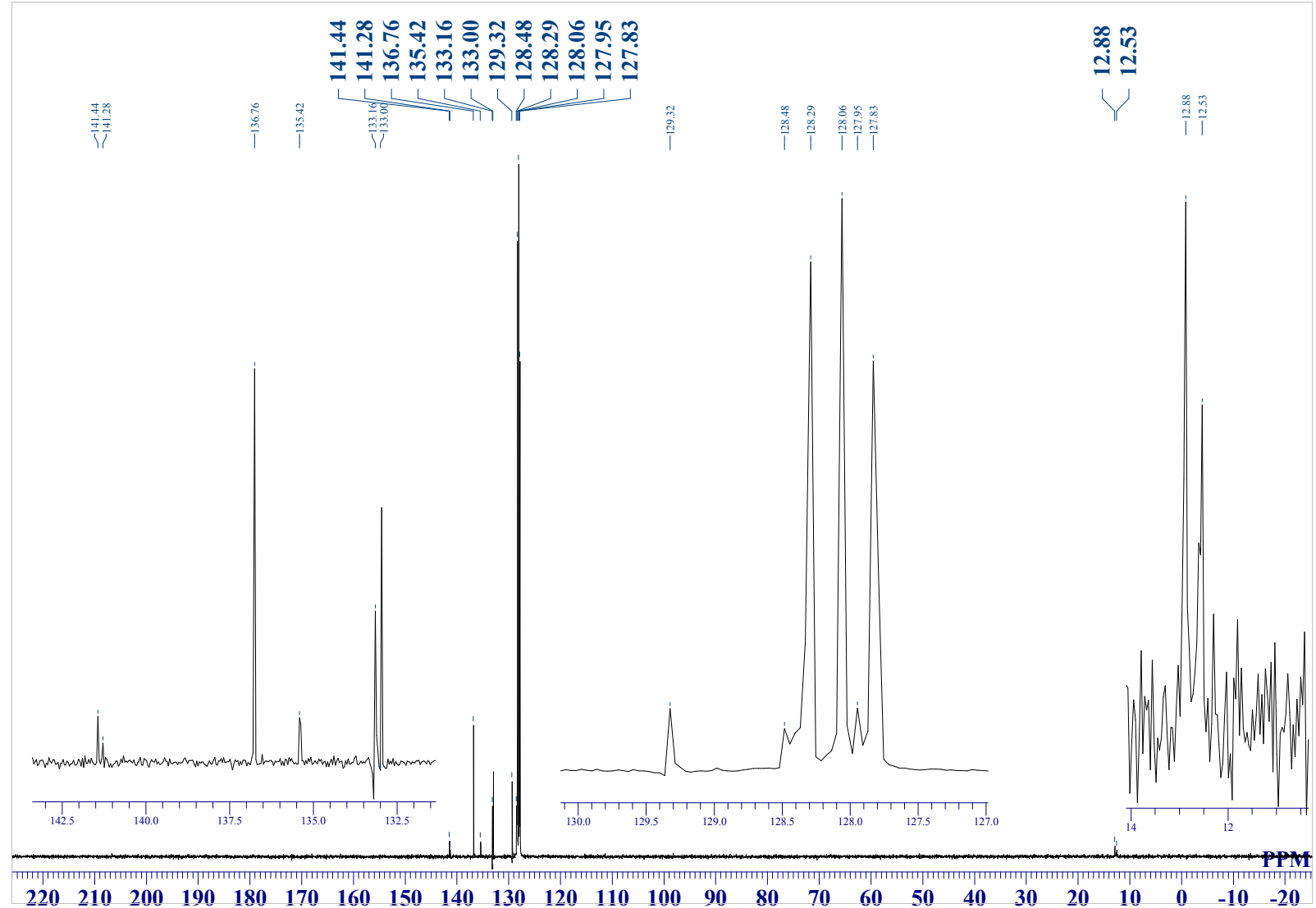


Figure S1-3. ${ }^{29} \mathrm{Si}$ NMR spectrum of solution of 2 in $\mathrm{C}_{6} \mathrm{D}_{6}$ at room temperature.

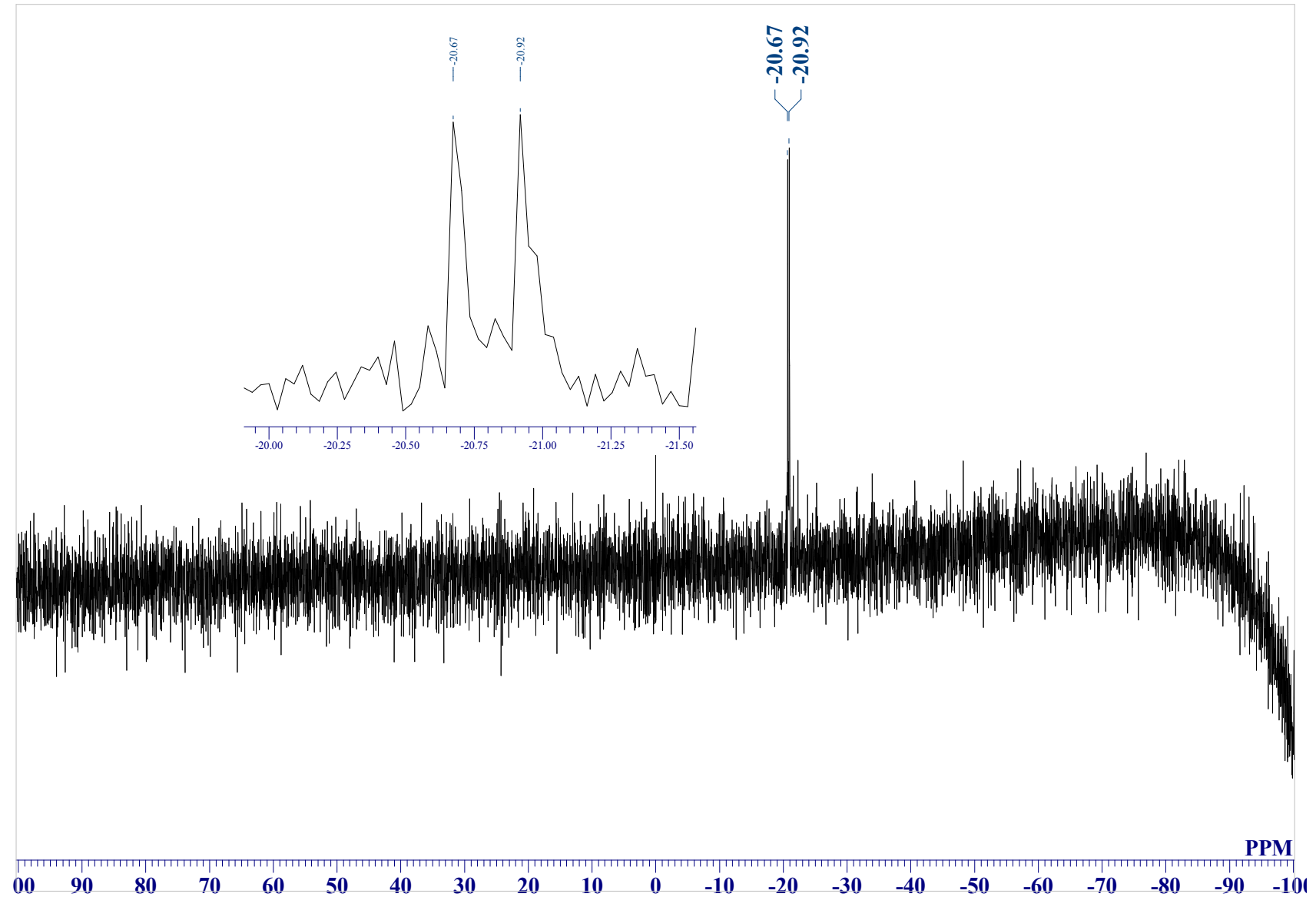

Figure S1-4. ${ }^{31} \mathrm{P}$ NMR spectrum of solution of 2 in $\mathrm{C}_{6} \mathrm{D}_{6}$ at room temperature.

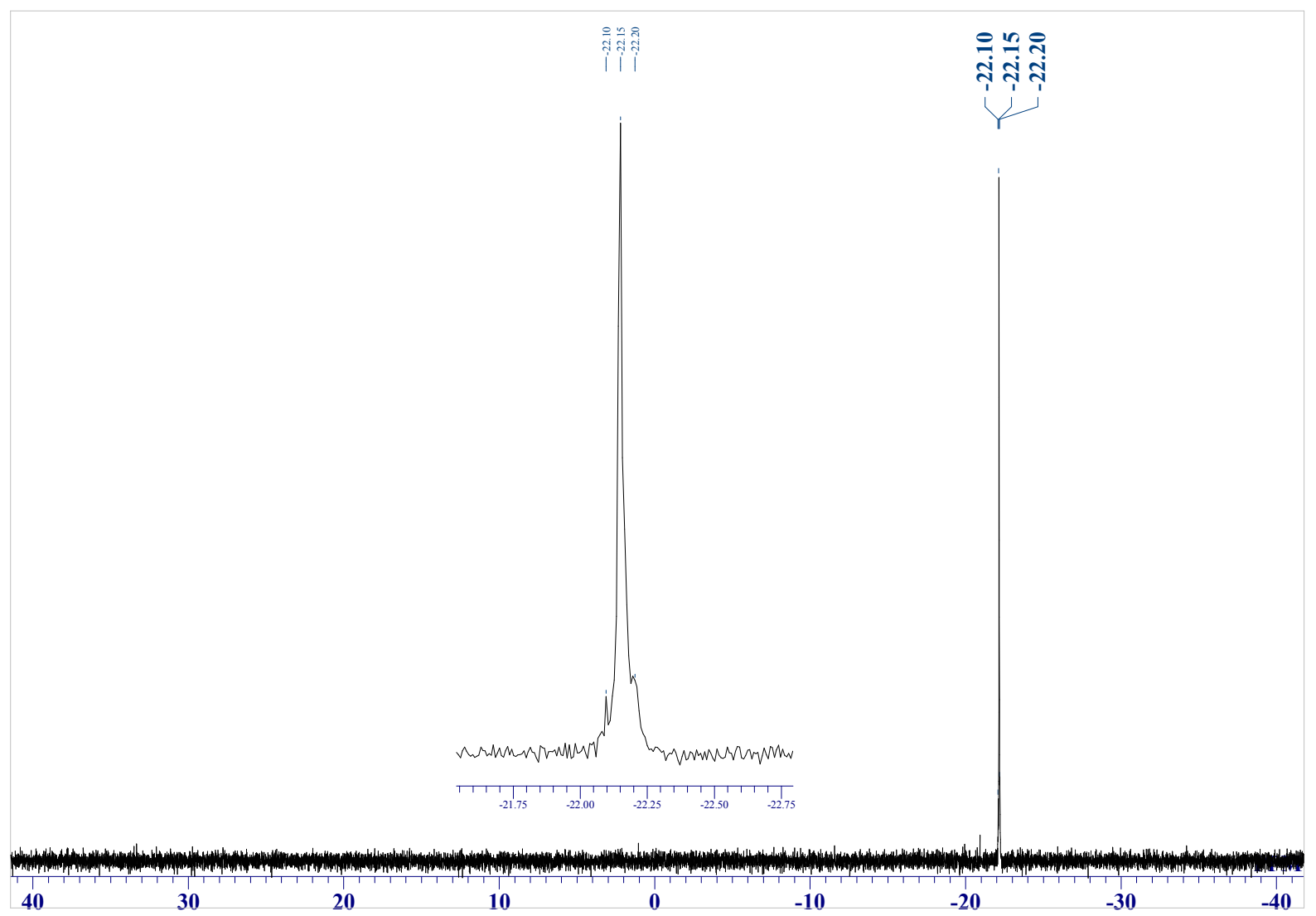


Figure S2-1. ${ }^{1} \mathrm{H}$ NMR spectrum of solution of 3 in $\mathrm{C}_{6} \mathrm{D}_{6}$ at room temperature.

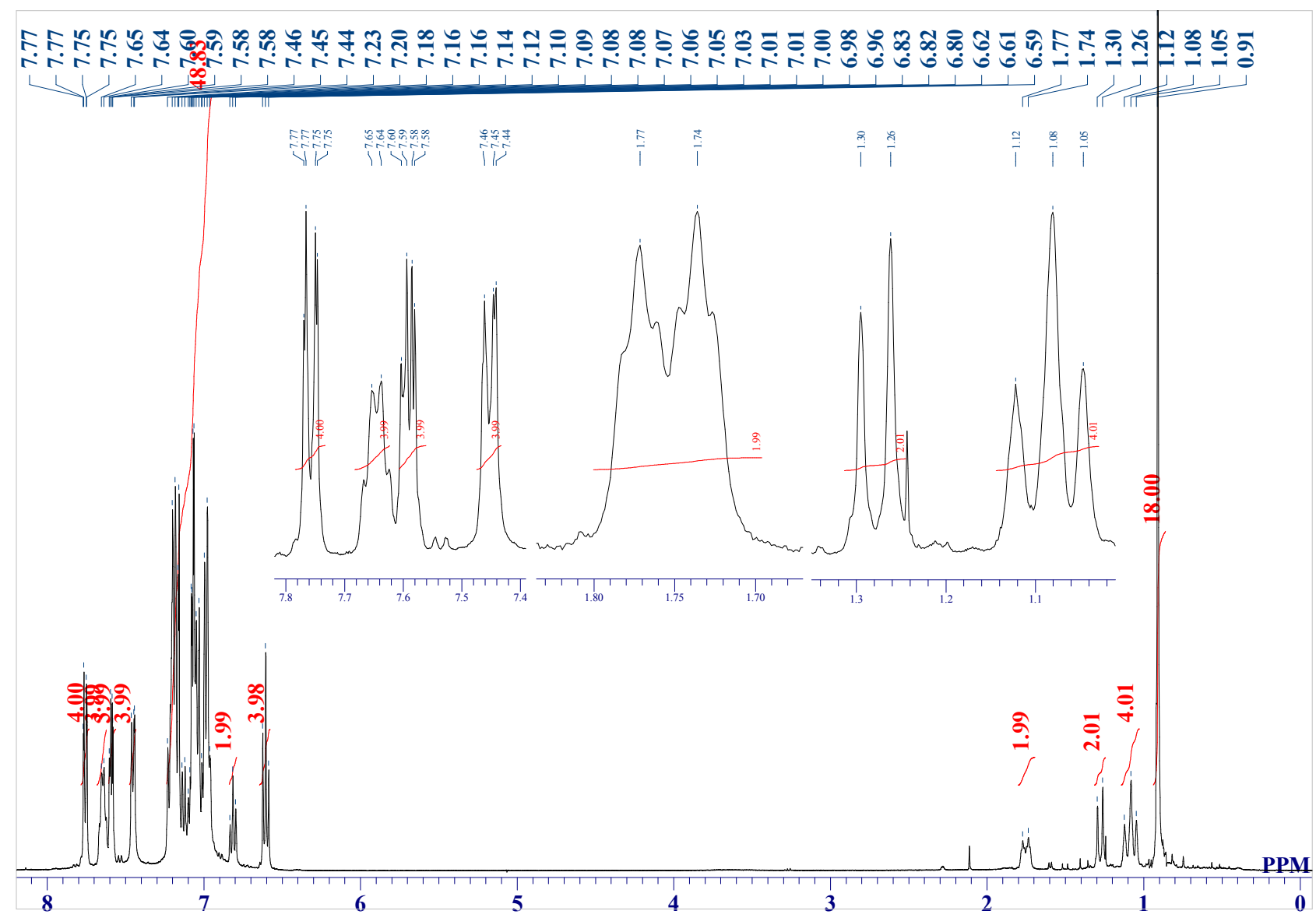

Figure S2-2. ${ }^{13} \mathrm{C}$ NMR spectrum of solution of 3 in $\mathrm{C}_{6} \mathrm{D}_{6}$ at room temperature.

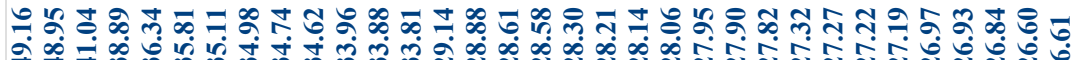

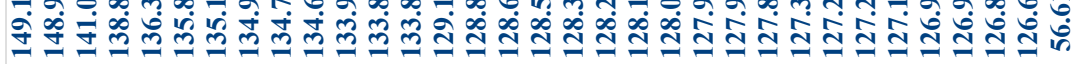

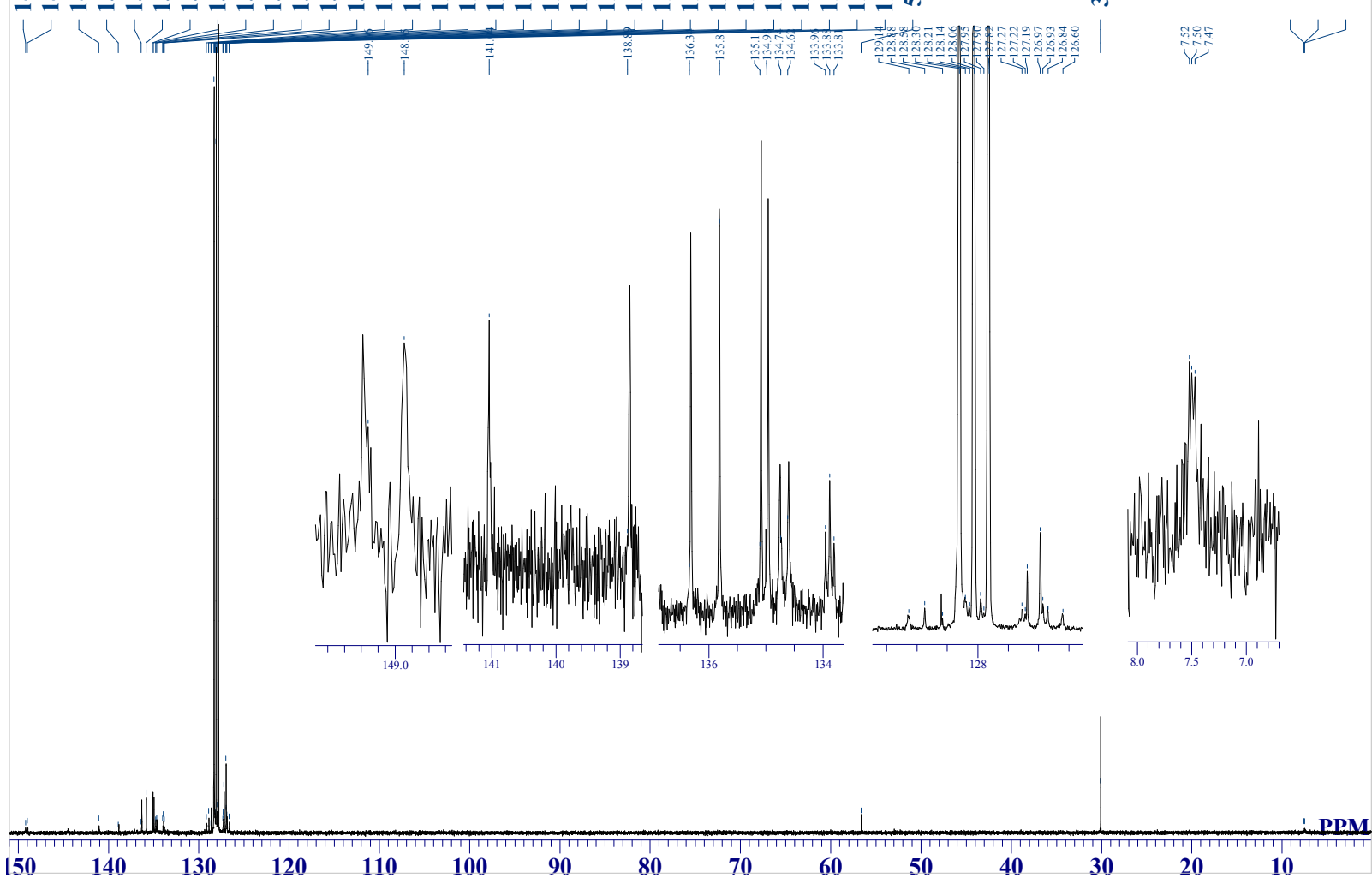


Figure S2-3. ${ }^{29} \mathrm{Si}$ NMR spectrum of solution of $\mathbf{3}$ in $\mathrm{C}_{6} \mathrm{D}_{6}$ at room temperature.

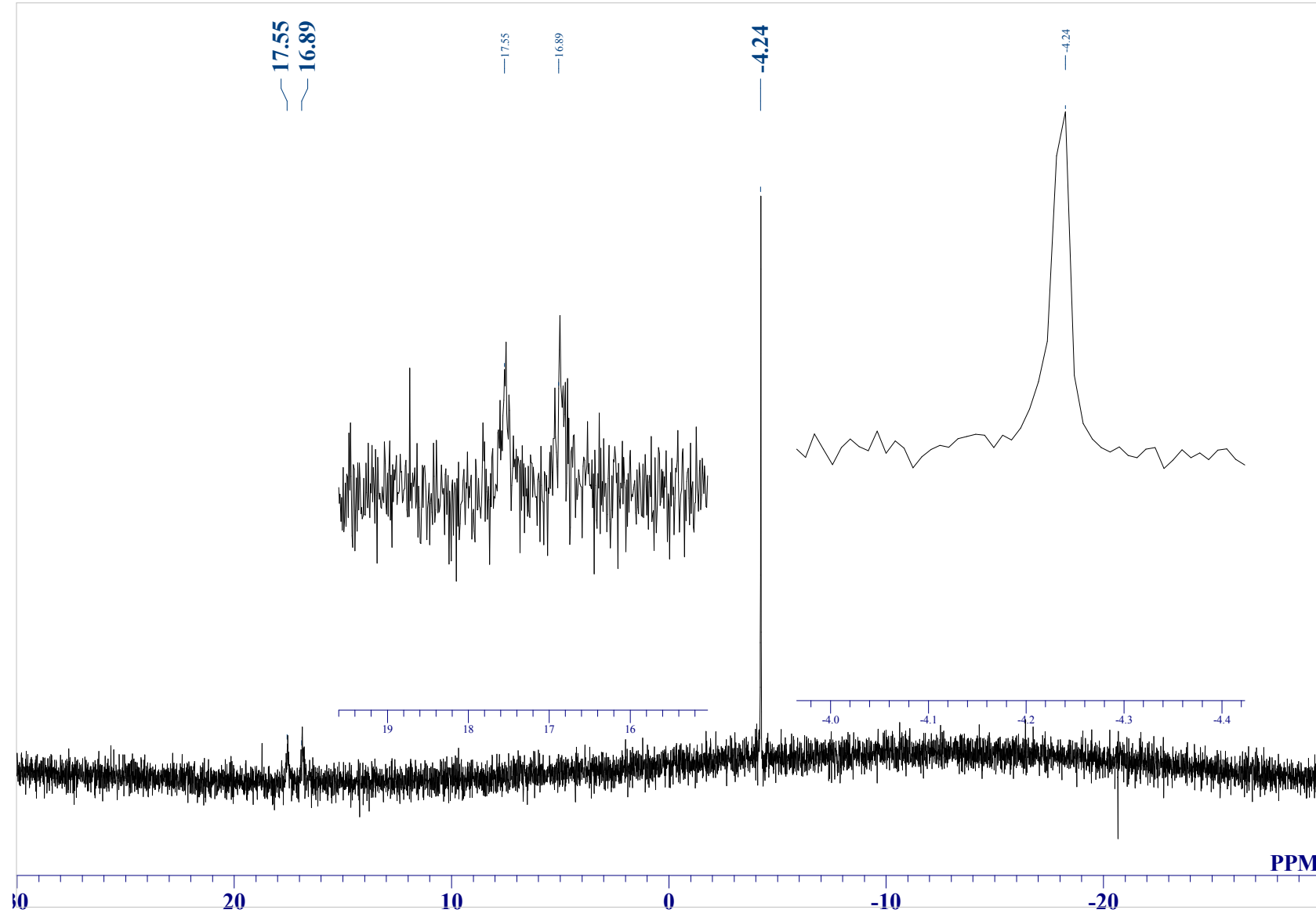

Figure S2-4. ${ }^{31} \mathrm{P}$ NMR spectrum of solution of 3 in $\mathrm{C}_{6} \mathrm{D}_{6}$ at room temperature.

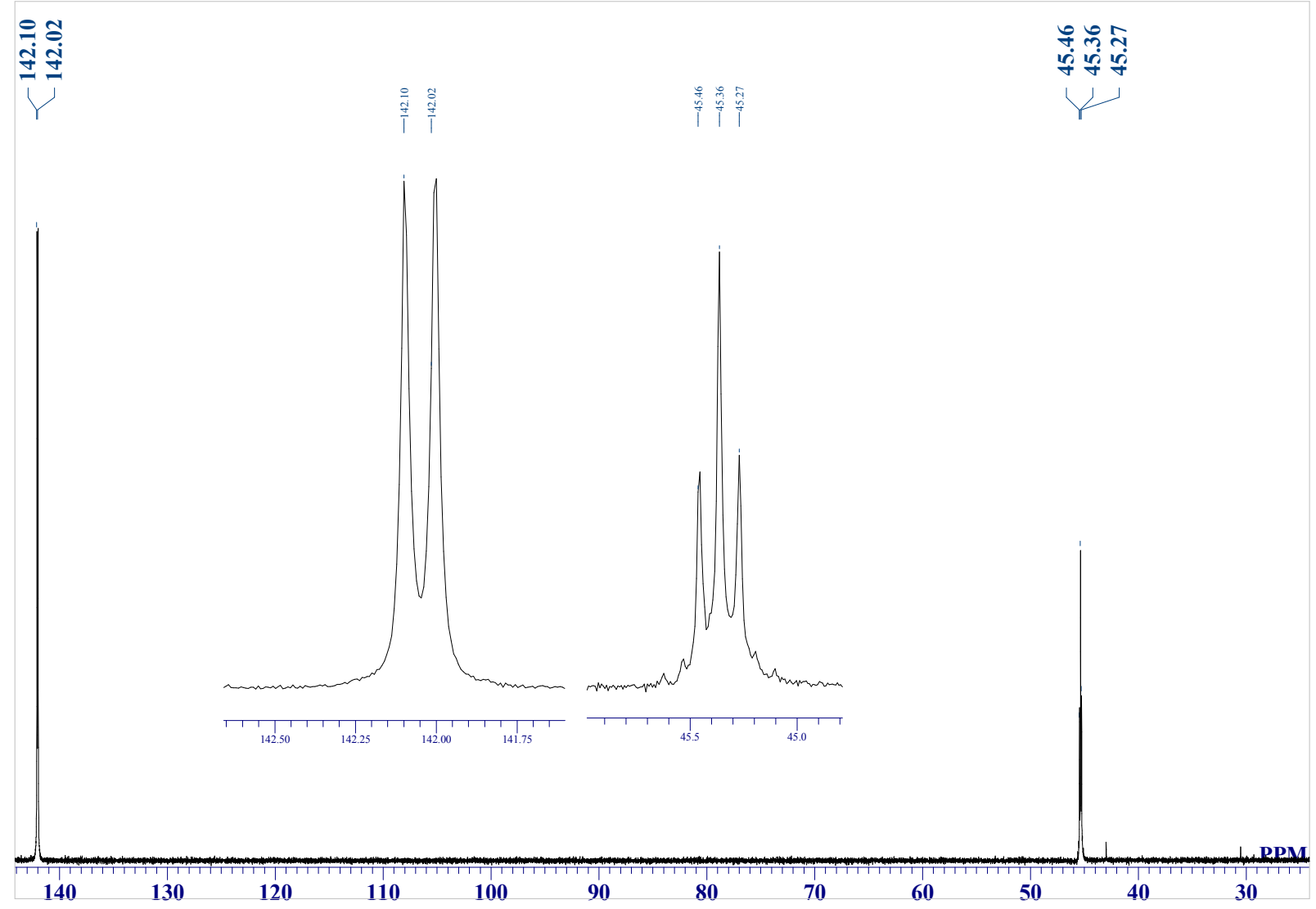

S-7 
Figure S3. ATR-IR spectrum of $\mathbf{3}$ in the solid state.

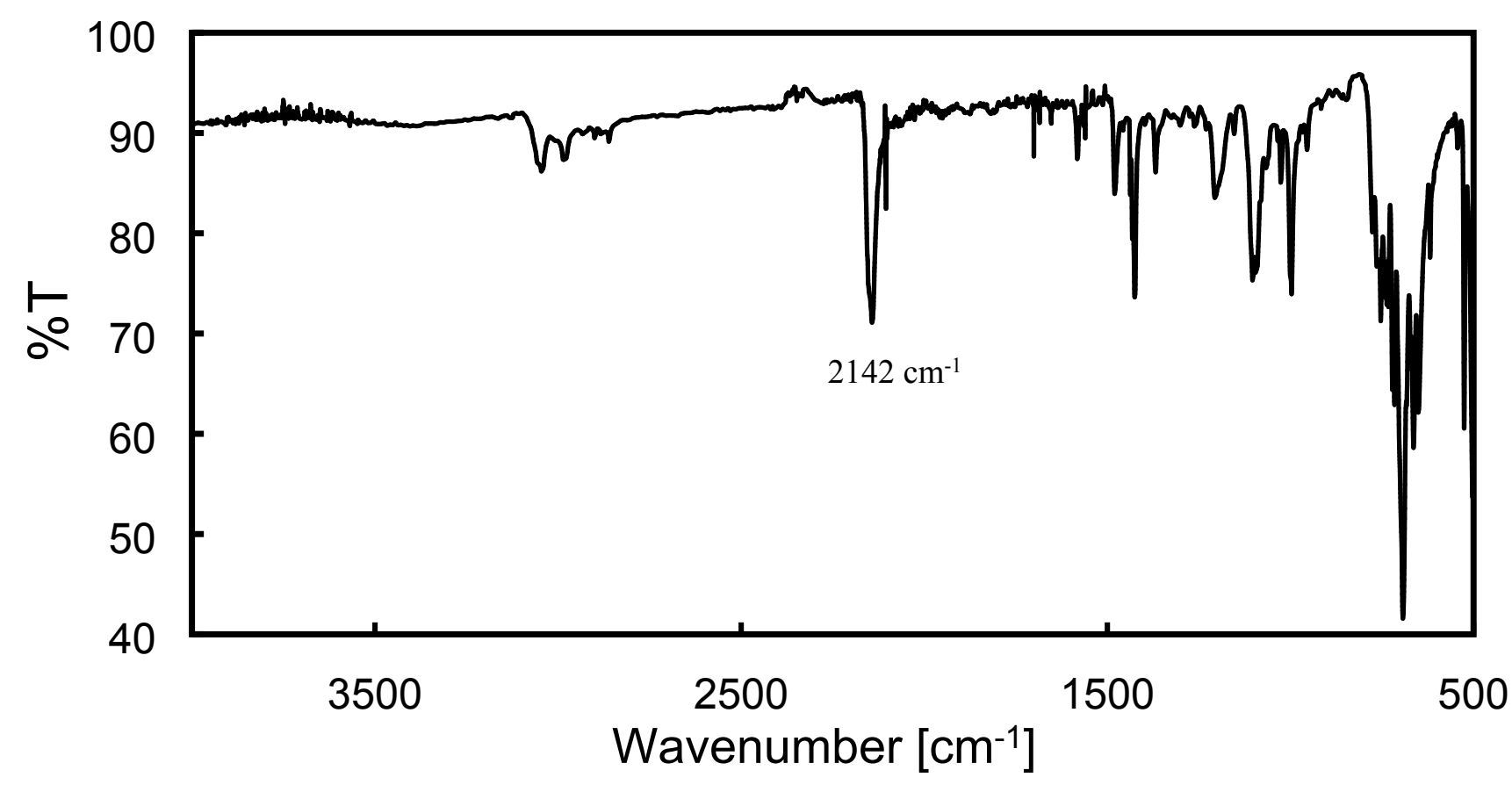


Figure S4-1. Comparison of the ${ }^{1} \mathrm{H}$ NMR data of isolated 3 and that of the crude product obtained from the reaction of disilane 2 with $\left[\mathrm{Pd}\left(\mathrm{CN}^{t} \mathrm{Bu}\right)_{2}\right]_{3}$ at $80^{\circ} \mathrm{C}$ in a molar ratio of $2: 4 / 3$ (molar ratio of 2 to " $\mathrm{Pd}\left(\mathrm{CN}^{t} \mathrm{Bu}\right)_{2}$ " was $2: 4)$.

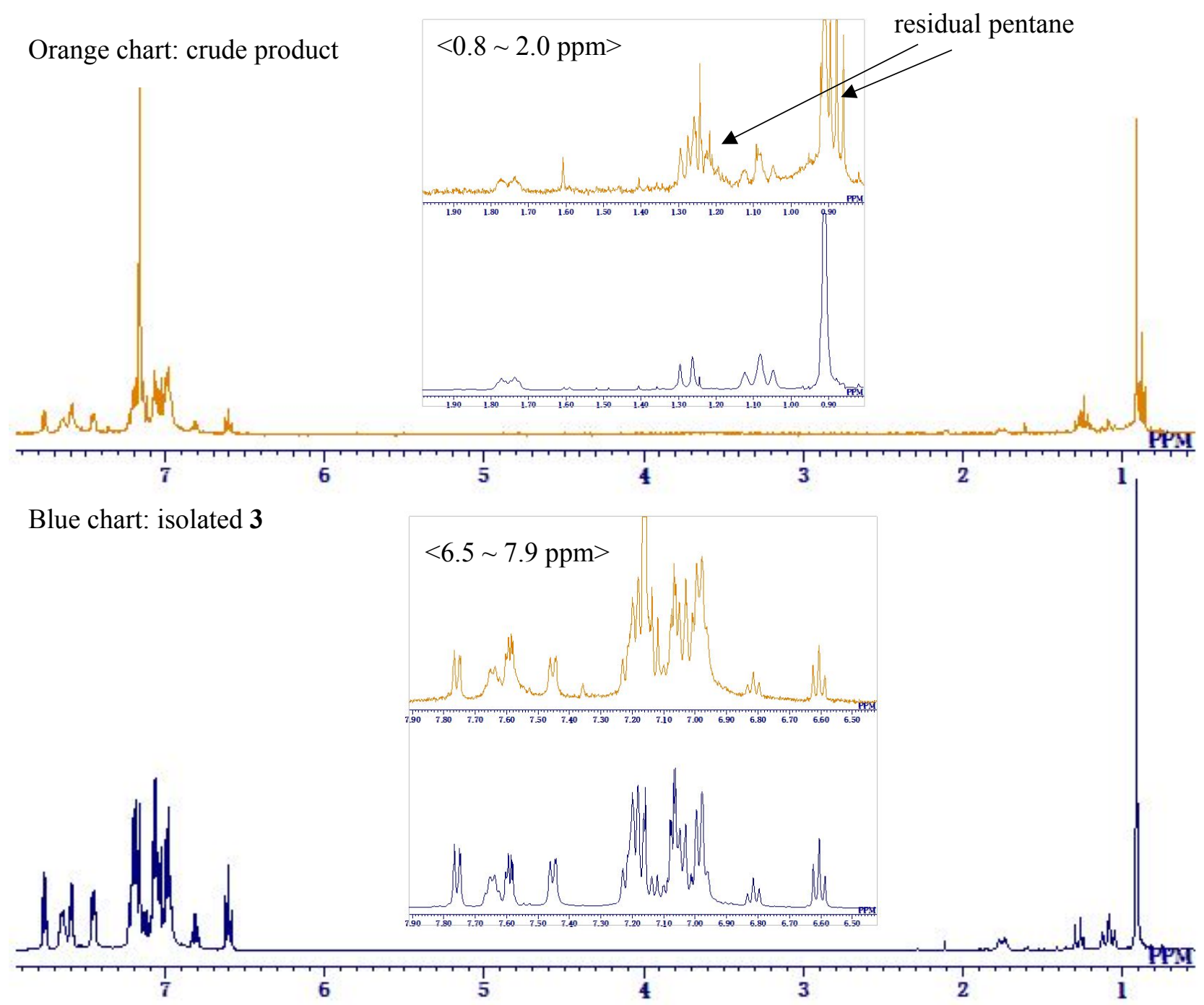


Figure S4-2. Comparison of the ${ }^{31} \mathrm{P}$ NMR data of isolated 3 and that of the crude product obtained from the reaction of disilane 2 with $\left[\mathrm{Pd}\left(\mathrm{CN}^{t} \mathrm{Bu}\right)_{2}\right]_{3}$ at $80^{\circ} \mathrm{C}$ in a molar ratio of $2: 4 / 3$ (molar ratio of 2 to " $\mathrm{Pd}\left(\mathrm{CN}^{t} \mathrm{Bu}\right)_{2}$ " was $2: 4)$.

Orange chart: crude product

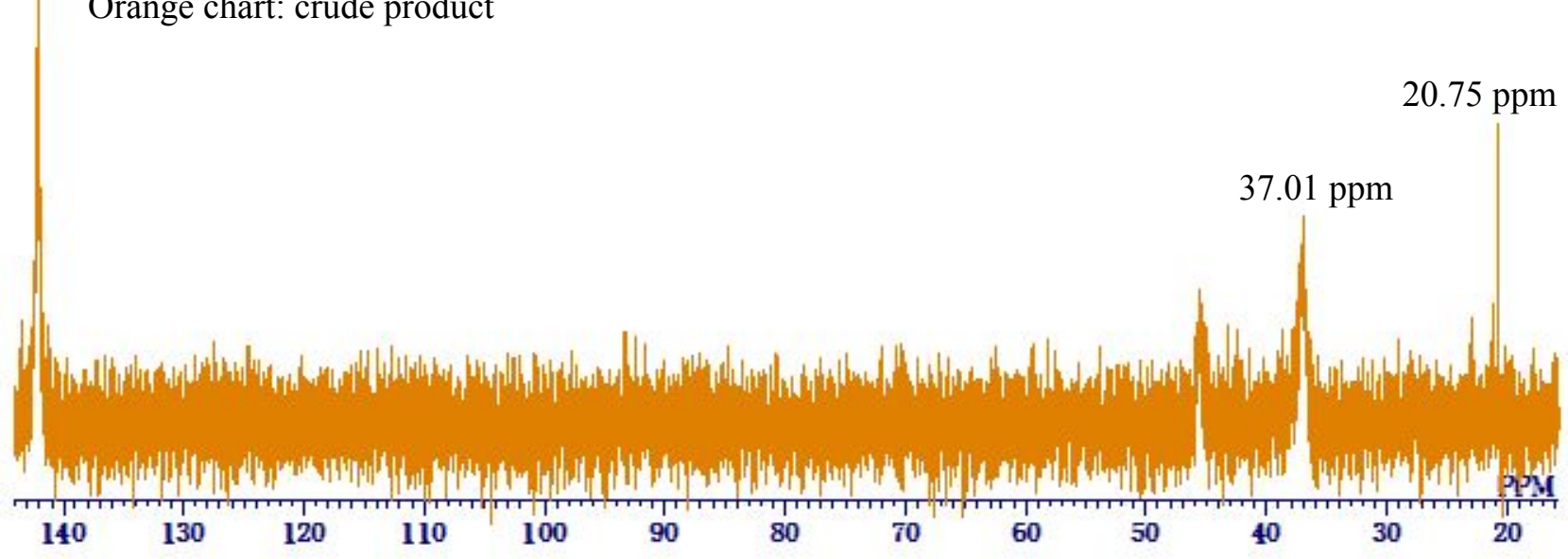

$142.06 \mathrm{ppm}$

Blue chart: isolated 3

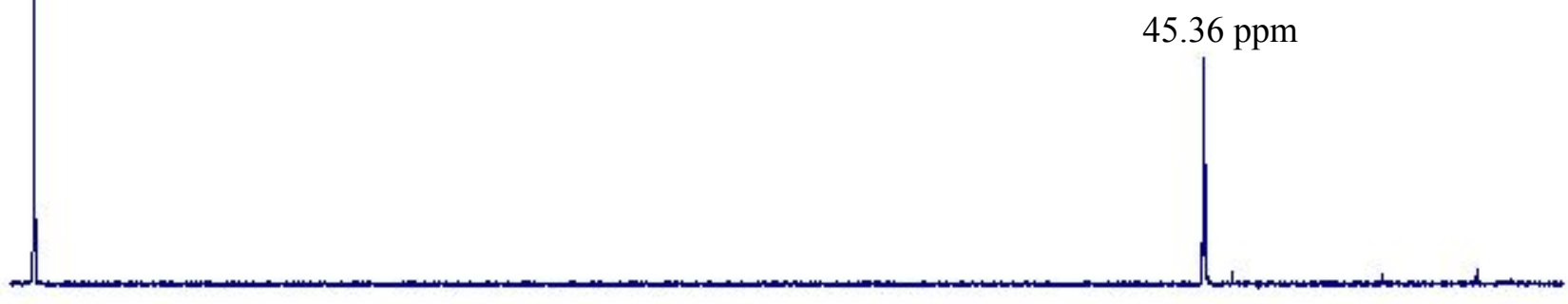

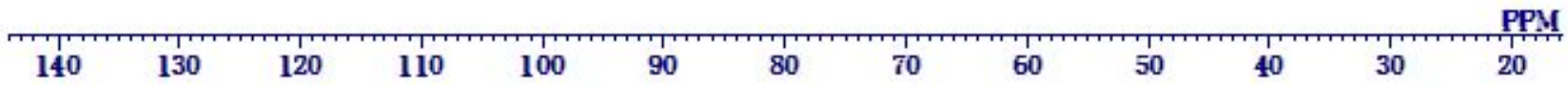


Figure S5. Comparison of the ${ }^{31} \mathrm{P}$ NMR data of the crude product obtained from the reaction of $\left[\mathrm{Pd}\left(\mathrm{CN} \mathrm{Bu}_{2}\right]_{3}\right.$ and $\mathrm{PPh}_{3}\left(\right.$ ratio of " $\mathrm{Pd}\left(\mathrm{CN}^{t} \mathrm{Bu}\right)_{2}$ " to $\mathrm{PPh}_{3}$ was $\left.1: 1\right)$ and that of the crude product of the reaction of disilane 2 with $\left[\mathrm{Pd}\left(\mathrm{CN}^{\prime} \mathrm{Bu}\right)_{2}\right]_{3}$ at $80^{\circ} \mathrm{C}$.

Orange chart: crude product of the reaction of $\left[\mathrm{Pd}\left(\mathrm{CN}{ }^{t} \mathrm{Bu}\right)_{2}\right]_{3}$ with $\mathrm{PPh}_{3}$

$35.78 \mathrm{ppm}$

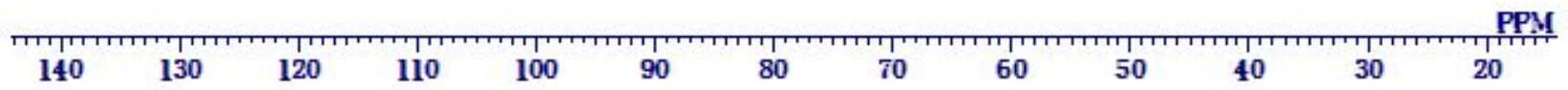

Blue chart: crude product of the reaction of disilane 2 and $\left[\mathrm{Pd}\left(\mathrm{CN}^{t} \mathrm{Bu}\right)_{2}\right]_{3}$

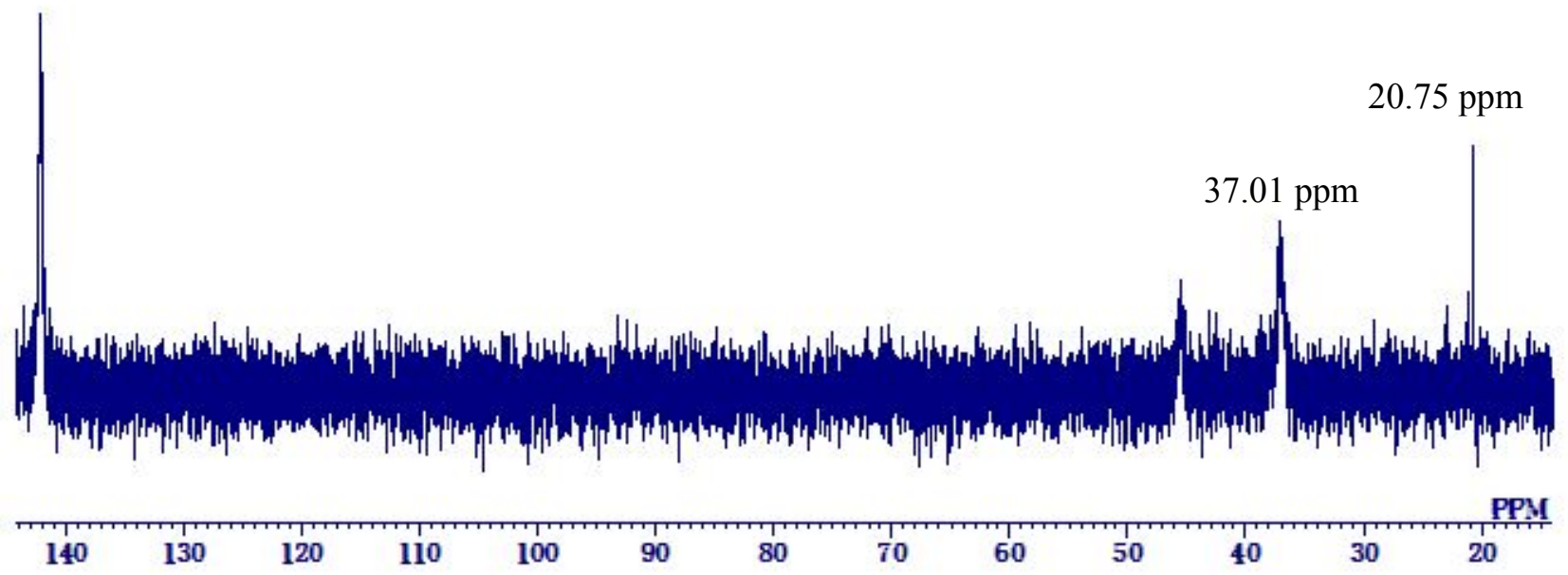




\section{Computational Details}

All of the calculations were performed using the Gaussian 09 program. ${ }^{3}$ Geometry optimization for complex 3 was carried out by using the DFT method with the PBE0 ${ }^{4}$, B3LYP , B3PW91 ${ }^{6}$ or $\mathrm{M}^{5} 6^{7}$ functional, and the selected bond distances for complex $\mathbf{3}$ (actual complex $\mathbf{3}$ and optimized $\mathbf{3}_{\text {opt }}$ ) were summarized in Figure S6. The calculated values of MBIs are summarized in Table S1. Natural Bond Orbital (NBO) analyses were performed using the NBO 3.1 program implemented in Gaussian 16. The effective core potentials and the basis set by the Stuttgart-Dresden-Bonn group ${ }^{8}$ and were used for $\mathrm{Pd}$ and the $6-31 \mathrm{G}^{* *}$ basis sets ${ }^{9}$ were used for C, $\mathrm{N}, \mathrm{Si}, \mathrm{P}$ and hydrogen atoms.

Table S1. Actual bond distances, calculated bond distances and Wiberg bond index for $\mathbf{3}$ and $\mathbf{3}_{\text {opt }}$.

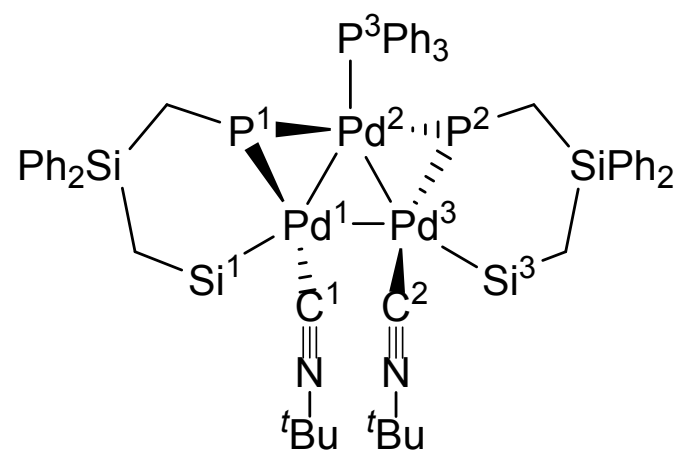

\begin{tabular}{|c|c|c|c|c|c|}
\hline & \multicolumn{2}{|c|}{$\begin{array}{c}\text { Actual bond } \\
\text { distances }(\AA)\end{array}$} & \multicolumn{2}{|l|}{ Estimated bond distances $(\AA)$} & \multicolumn{2}{l|}{ Wiberg bond index } \\
& $\mathrm{XRD}$ & $\mathrm{B} 3 \mathrm{PW} 91$ & $\mathrm{M} 06$ & $\mathrm{~B} 3 \mathrm{PW} 91$ & $\mathrm{M} 06$ \\
\hline $\mathrm{Pd}(1)-\mathrm{Pd}(2)$ & $2.9106(8)$ & 2.95627 & 2.96232 & 0.2036 & 0.1955 \\
\hline $\mathrm{Pd}(1)-\mathrm{Pd}(3)$ & $2.9340(6)$ & 2.92589 & 2.99022 & 0.1896 & 0.1906 \\
\hline $\operatorname{Pd}(2)-\mathrm{Pd}(3)$ & $2.8972(6)$ & 3.00007 & 2.99983 & 0.1900 & 0.1857 \\
\hline $\operatorname{Pd}(1)-\operatorname{Si}(1)$ & $2.3331(7)$ & 2.37535 & 2.38757 & 0.5539 & 0.5139 \\
\hline $\operatorname{Pd}(1)-\mathrm{P}(1)$ & $2.2307(6)$ & 2.27495 & 2.28764 & 0.6063 & 0.5707 \\
\hline $\operatorname{Pd}(1)-\mathrm{C}(1)$ & $1.9790(17)$ & 1.97584 & 2.00077 & 0.5812 & 0.5471 \\
\hline $\operatorname{Pd}(2)-\mathrm{P}(1)$ & $2.2871(7)$ & 2.34044 & 2.35139 & 0.4169 & 0.3809 \\
\hline $\operatorname{Pd}(2)-\mathrm{P}(2)$ & $2.2811(6)$ & 2.33173 & 2.34554 & 0.4285 & 0.3869 \\
\hline $\operatorname{Pd}(2)-\mathrm{P}(3)$ & $2.2874(7)$ & 2.34627 & 2.35613 & 0.4094 & 0.3568 \\
\hline $\operatorname{Pd}(3)-\mathrm{P}(2)$ & $2.2184(7)$ & 2.26532 & 2.27908 & 0.5723 & 0.5581 \\
\hline $\operatorname{Pd}(3)-\operatorname{Si}(3)$ & $2.3326(6)$ & 2.38093 & 2.39084 & 0.5354 & 0.5068 \\
\hline $\operatorname{Pd}(3)-\mathrm{C}(2)$ & $1.9815(17)$ & 1.98362 & 2.00869 & 0.5600 & 0.5337 \\
\hline
\end{tabular}


Figure S6. Optimized molecular structure of $3\left(\mathbf{3}_{\text {opt }}\right)$ with B3PW91 (upper) and with M06 (lower). The orange balls are palladium atoms, the blue balls are silicon atoms, yellow balls are phosphorus atoms, the pale blue balls are nitrogen atoms, the grey ones are carbon atoms.
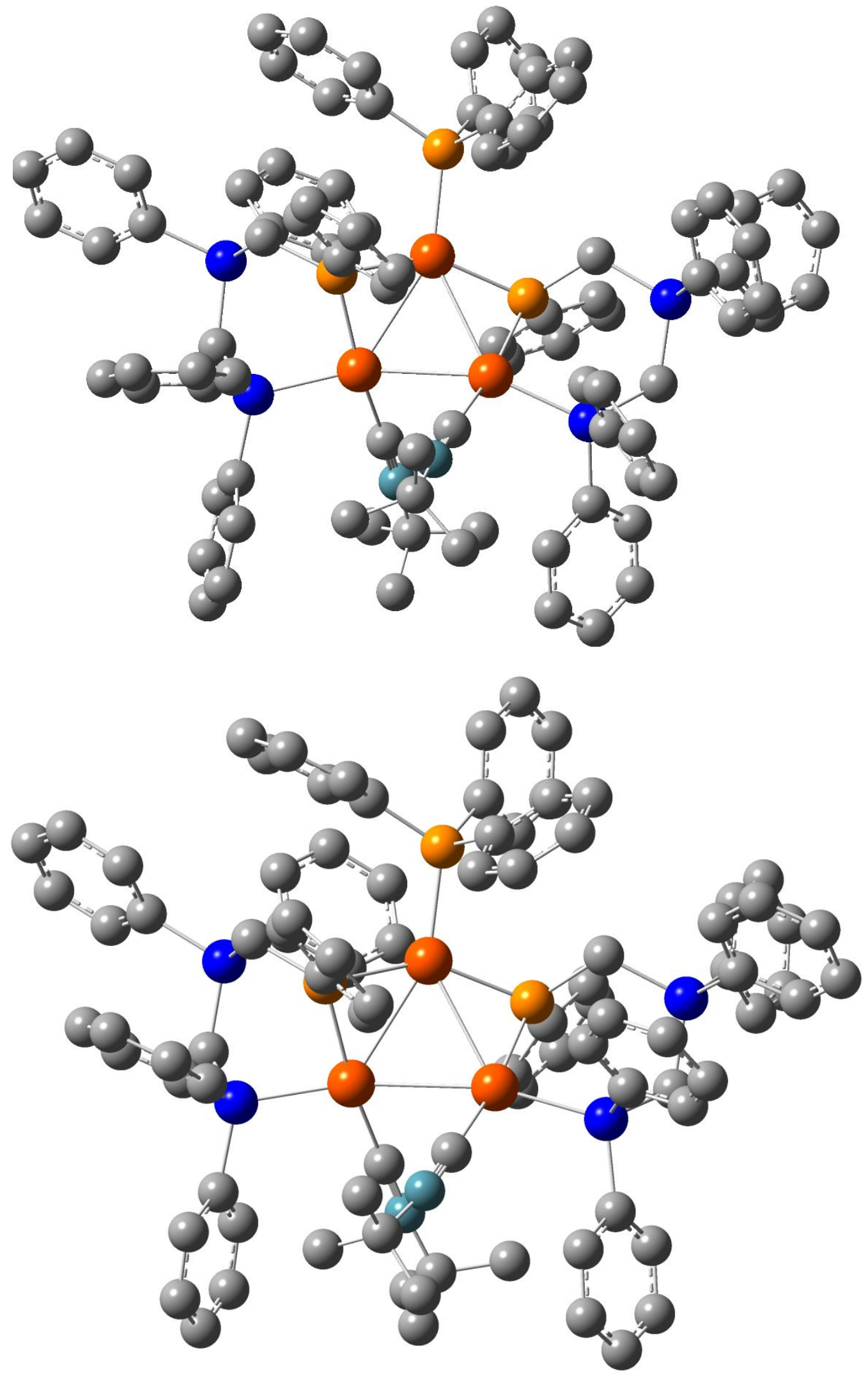
Table S2. The DFT-optimized Geometry for complex $\mathbf{3}_{\text {opt }}$ (in XYZ format) with B3PW91 functional

\begin{tabular}{|c|c|c|c|c|c|c|c|}
\hline & 1.580000 & 1.298000 & -0.221000 & $\mathrm{C}$ & 7.610000 & -1.246000 & -1.259000 \\
\hline$P$ & 0.219000 & -1.316000 & 0.012000 & $\mathrm{C}$ & 8.916000 & -1.687000 & -1.052000 \\
\hline$P_{0}$ & -1.333000 & 1.251000 & 0.046000 & $\mathrm{C}$ & 9.208000 & -2.516000 & 0.030000 \\
\hline$p$ & 0.184000 & -3.627000 & 0.417000 & $\mathrm{C}$ & 8.187000 & -2.901000 & 0.896000 \\
\hline 1 & 2.149000 & -0.499000 & 1.053000 & $\mathrm{C}$ & 6.882000 & -2.459000 & 0.680000 \\
\hline$P$ & -1.867000 & -0.754000 & -0.864000 & $\mathrm{C}$ & 4.057000 & -2.080000 & -2.109000 \\
\hline $\mathrm{Si}$ & 4.794000 & -1.010000 & -0.732000 & $\mathrm{C}$ & 4.781000 & -3.129000 & -2.698000 \\
\hline $\mathrm{Si}$ & 3.839000 & 2.032000 & -0.267000 & $\mathrm{C}$ & 4.234000 & -3.902000 & -3.724000 \\
\hline & -5.053000 & -0.840000 & -0.230000 & $\mathrm{C}$ & 2.945000 & -3.639000 & -4.185000 \\
\hline & -3.567000 & 2.063000 & -0.099000 & $\mathrm{C}$ & 2.208000 & -2.598000 & -3.617000 \\
\hline $\mathrm{N}$ & 0.747000 & 3.167000 & -2.610000 & $\mathrm{C}$ & 2.761000 & -1.828000 & -2.594000 \\
\hline $\mathrm{N}$ & -0.483000 & 3.652000 & 1.904000 & $\mathrm{C}$ & -4.919000 & 0.926000 & -0.831000 \\
\hline & 1.011000 & 2.493000 & -1.688000 & $\mathrm{C}$ & -3.328000 & -1.658000 & -0.179000 \\
\hline & 0.452000 & 4.015000 & -3.735000 & $\mathrm{C}$ & 1.884000 & -0.436000 & 2.873000 \\
\hline & 0.615000 & 5.472000 & -3.280000 & $\mathrm{C}$ & 0.728000 & 0.197000 & 3.363000 \\
\hline $\mathrm{C}$ & 1.451000 & 3.680000 & -4.851000 & $\mathrm{C}$ & 0.446000 & 0.220000 & 4.726000 \\
\hline & -0.988000 & 3.741000 & -4.186000 & $\mathrm{C}$ & 1.312000 & -0.392000 & 5.636000 \\
\hline $\mathrm{C}$ & -0.735000 & 2.775000 & 1.165000 & $\mathrm{C}$ & 2.457000 & -1.028000 & 5.163000 \\
\hline $\mathrm{C}$ & -0.265000 & 4.703000 & 2.864000 & $\mathrm{C}$ & 2.741000 & -1.052000 & 3.797000 \\
\hline $\mathrm{C}$ & 1.122000 & 5.309000 & 2.618000 & $\mathrm{C}$ & -3.683000 & 3.649000 & -1.172000 \\
\hline & -0.352000 & 4.082000 & 4.265000 & $\mathrm{C}$ & -2.955000 & 4.801000 & -0.823000 \\
\hline $\mathrm{C}$ & -1.361000 & 5.762000 & 2.680000 & $\mathrm{C}$ & -3.063000 & 5.989000 & -1.545000 \\
\hline & 4.850000 & 0.788000 & -1.300000 & $\mathrm{C}$ & -3.901000 & 6.056000 & -2.659000 \\
\hline & 3.845000 & -1.215000 & 0.903000 & $\mathrm{C}$ & -4.625000 & 4.928000 & -3.035000 \\
\hline & 4.120000 & 3.731000 & -1.105000 & $\mathrm{C}$ & -4.517000 & 3.746000 & -2.299000 \\
\hline & 3.925000 & 4.921000 & -0.381000 & $\mathrm{C}$ & -4.183000 & 2.599000 & 1.630000 \\
\hline & 4.143000 & 6.174000 & -0.952000 & $\mathrm{C}$ & -3.757000 & 1.931000 & 2.790000 \\
\hline & 4.574000 & 6.274000 & -2.276000 & $\mathrm{C}$ & -4.224000 & 2.291000 & 4.054000 \\
\hline & 4.781000 & 5.110000 & -3.015000 & $\mathrm{C}$ & -5.129000 & 3.344000 & 4.190000 \\
\hline & 4.554000 & 3.860000 & -2.435000 & $\mathrm{C}$ & -5.564000 & 4.026000 & 3.054000 \\
\hline & 4.723000 & 2.261000 & 1.412000 & $\mathrm{C}$ & -5.097000 & 3.654000 & 1.793000 \\
\hline & 4.019000 & 2.345000 & 2.623000 & $\mathrm{C}$ & -5.841000 & -0.981000 & 1.485000 \\
\hline & 4.672000 & 2.572000 & 3.837000 & $\mathrm{C}$ & -5.573000 & -2.078000 & 2.321000 \\
\hline$c$ & 6.057000 & 2.715000 & 3.865000 & $\mathrm{C}$ & -6.216000 & -2.229000 & 3.549000 \\
\hline$C$ & 6.782000 & 2.634000 & 2.676000 & $\mathrm{C}$ & -7.146000 & -1.281000 & 3.971000 \\
\hline & 6.120000 & 2.416000 & 1.469000 & $\mathrm{C}$ & -7.426000 & -0.183000 & 3.160000 \\
\hline & 6.561000 & -1.619000 & -0.400000 & $\mathrm{C}$ & -6.781000 & -0.039000 & 1.933000 \\
\hline
\end{tabular}




\begin{tabular}{|c|c|c|c|c|c|c|c|}
\hline $\mathrm{C}$ & -6.166000 & -1.835000 & -1.410000 & $\mathrm{H}$ & 1.289000 & 6.129000 & 3.322000 \\
\hline $\mathrm{C}$ & -6.287000 & -3.229000 & -1.272000 & $\mathrm{H}$ & 1.907000 & 4.562000 & 2.761000 \\
\hline$C$ & -7.113000 & -3.975000 & -2.111000 & $\mathrm{H}$ & 1.198000 & 5.702000 & 1.601000 \\
\hline $\mathrm{C}$ & -7.847000 & -3.339000 & -3.112000 & $\mathrm{H}$ & -0.170000 & 4.857000 & 5.016000 \\
\hline $\mathrm{C}$ & -7.748000 & -1.958000 & -3.266000 & $\mathrm{H}$ & -1.345000 & 3.657000 & 4.434000 \\
\hline $\mathrm{C}$ & -6.916000 & -1.219000 & -2.424000 & $\mathrm{H}$ & 0.395000 & 3.294000 & 4.388000 \\
\hline & -2.055000 & -0.813000 & -2.685000 & $\mathrm{H}$ & -1.239000 & 6.543000 & 3.437000 \\
\hline C & -1.030000 & -0.258000 & -3.472000 & $\mathrm{H}$ & -1.290000 & 6.230000 & 1.694000 \\
\hline$C$ & -1.086000 & -0.317000 & -4.861000 & $\mathrm{H}$ & -2.355000 & 5.320000 & 2.789000 \\
\hline & -2.168000 & -0.929000 & -5.499000 & $\mathrm{H}$ & 4.461000 & 0.820000 & -2.325000 \\
\hline 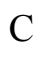 & -3.188000 & -1.484000 & -4.731000 & $\mathrm{H}$ & 5.896000 & 1.117000 & -1.347000 \\
\hline $\mathrm{C}$ & -3.132000 & -1.432000 & -3.337000 & $\mathrm{H}$ & 3.800000 & -2.281000 & 1.158000 \\
\hline $\mathrm{C}$ & -0.293000 & -4.795000 & -0.929000 & $\mathrm{H}$ & 4.474000 & -0.715000 & 1.654000 \\
\hline & -0.024000 & -6.171000 & -0.855000 & $\mathrm{H}$ & 3.614000 & 4.864000 & 0.660000 \\
\hline 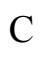 & -0.429000 & -7.025000 & -1.878000 & $\mathrm{H}$ & 3.991000 & 7.074000 & -0.361000 \\
\hline $\mathrm{C}$ & -1.104000 & -6.519000 & -2.990000 & $\mathrm{H}$ & 4.756000 & 7.248000 & -2.722000 \\
\hline & -1.365000 & -5.154000 & -3.077000 & $\mathrm{H}$ & 5.132000 & 5.174000 & -4.043000 \\
\hline$c$ & -0.957000 & -4.297000 & -2.055000 & $\mathrm{H}$ & 4.737000 & 2.969000 & -3.031000 \\
\hline $\mathrm{C}$ & -1.021000 & -3.992000 & 1.768000 & $\mathrm{H}$ & 2.940000 & 2.210000 & 2.614000 \\
\hline $\mathrm{C}$ & -1.108000 & -3.075000 & 2.826000 & $\mathrm{H}$ & 4.098000 & 2.624000 & 4.759000 \\
\hline$C$ & -1.967000 & -3.309000 & 3.898000 & $\mathrm{H}$ & 6.571000 & 2.885000 & 4.808000 \\
\hline $\mathrm{C}$ & -2.766000 & -4.452000 & 3.919000 & $\mathrm{H}$ & 7.864000 & 2.741000 & 2.688000 \\
\hline $\mathrm{C}$ & -2.703000 & -5.357000 & 2.860000 & $\mathrm{H}$ & 6.707000 & 2.369000 & 0.554000 \\
\hline $\mathrm{C}$ & -1.834000 & -5.132000 & 1.792000 & $\mathrm{H}$ & 7.406000 & -0.596000 & -2.108000 \\
\hline $\mathrm{C}$ & 1.730000 & -4.428000 & 1.033000 & $\mathrm{H}$ & 9.706000 & -1.381000 & -1.734000 \\
\hline$C$ & 1.924000 & -4.770000 & 2.376000 & $\mathrm{H}$ & 10.226000 & -2.858000 & 0.198000 \\
\hline 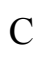 & 3.130000 & -5.335000 & 2.795000 & $\mathrm{H}$ & 8.407000 & -3.545000 & 1.745000 \\
\hline $\mathrm{C}$ & 4.153000 & -5.568000 & 1.879000 & $\mathrm{H}$ & 6.103000 & -2.776000 & 1.369000 \\
\hline $\mathrm{C}$ & 3.968000 & -5.227000 & 0.538000 & $\mathrm{H}$ & 5.790000 & -3.345000 & -2.355000 \\
\hline & 2.770000 & -4.656000 & 0.118000 & $\mathrm{H}$ & 4.816000 & -4.708000 & -4.164000 \\
\hline $\mathrm{H}$ & 0.414000 & 6.136000 & -4.125000 & $\mathrm{H}$ & 2.515000 & -4.241000 & -4.981000 \\
\hline $\mathrm{H}$ & -0.097000 & 5.703000 & -2.483000 & $\mathrm{H}$ & 1.201000 & -2.387000 & -3.966000 \\
\hline & 1.631000 & 5.656000 & -2.919000 & $\mathrm{H}$ & 2.164000 & -1.027000 & -2.158000 \\
\hline 11 & 1.242000 & 4.309000 & -5.722000 & $\mathrm{H}$ & -5.890000 & 1.432000 & -0.737000 \\
\hline $\mathrm{H}$ & 2.475000 & 3.867000 & -4.519000 & $\mathrm{H}$ & -4.704000 & 0.848000 & -1.907000 \\
\hline 11 & 1.362000 & 2.632000 & -5.151000 & $\mathrm{H}$ & -3.061000 & -1.805000 & 0.874000 \\
\hline 11 & -1.224000 & 4.380000 & -5.042000 & $\mathrm{H}$ & -3.355000 & -2.661000 & -0.625000 \\
\hline $\mathrm{H}$ & -1.111000 & 2.697000 & -4.486000 & $\mathrm{H}$ & 0.046000 & 0.665000 & 2.657000 \\
\hline & -1.697000 & 3.957000 & -3.384000 & $\mathrm{H}$ & -0.454000 & 0.717000 & 5.080000 \\
\hline
\end{tabular}




$\begin{array}{rrrrrrrr}\mathrm{H} & 1.094000 & -0.374000 & 6.700000 & \mathrm{H} & -6.851000 & -0.142000 & -2.563000 \\ \mathrm{H} & 3.138000 & -1.512000 & 5.858000 & \mathrm{H} & -0.186000 & 0.217000 & -2.978000 \\ \mathrm{H} & 3.637000 & -1.560000 & 3.457000 & \mathrm{H} & -0.279000 & 0.111000 & -5.451000 \\ \mathrm{H} & -2.294000 & 4.769000 & 0.038000 & \mathrm{H} & -2.210000 & -0.974000 & -6.584000 \\ \mathrm{H} & -2.496000 & 6.865000 & -1.238000 & \mathrm{H} & -4.038000 & -1.962000 & -5.212000 \\ \mathrm{H} & -3.989000 & 6.980000 & -3.225000 & \mathrm{H} & -3.937000 & -1.881000 & -2.766000 \\ \mathrm{H} & -5.283000 & 4.966000 & -3.900000 & \mathrm{H} & 0.511000 & -6.573000 & 0.001000 \\ \mathrm{H} & -5.106000 & 2.888000 & -2.610000 & \mathrm{H} & -0.214000 & -8.088000 & -1.808000 \\ \mathrm{H} & -3.045000 & 1.113000 & 2.697000 & \mathrm{H} & -1.416000 & -7.187000 & -3.787000 \\ \mathrm{H} & -3.889000 & 1.746000 & 4.933000 & \mathrm{H} & -1.879000 & -4.745000 & -3.943000 \\ \mathrm{H} & -5.493000 & 3.630000 & 5.174000 & \mathrm{H} & -1.138000 & -3.230000 & -2.135000 \\ \mathrm{H} & -6.269000 & 4.849000 & 3.150000 & \mathrm{H} & -0.500000 & -2.173000 & 2.807000 \\ \mathrm{H} & -5.446000 & 4.201000 & 0.919000 & \mathrm{H} & -2.014000 & -2.592000 & 4.713000 \\ \mathrm{H} & -4.847000 & -2.831000 & 2.021000 & \mathrm{H} & -3.437000 & -4.634000 & 4.754000 \\ \mathrm{H} & -5.988000 & -3.088000 & 4.176000 & \mathrm{H} & -3.328000 & -6.246000 & 2.864000 \\ \mathrm{H} & -7.648000 & -1.396000 & 4.929000 & \mathrm{H} & -1.794000 & -5.847000 & 0.977000 \\ \mathrm{H} & -8.143000 & 0.567000 & 3.485000 & \mathrm{H} & 1.132000 & -4.605000 & 3.100000 \\ \mathrm{H} & -7.008000 & 0.832000 & 1.324000 & \mathrm{H} & 3.262000 & -5.599000 & 3.841000 \\ \mathrm{H} & -5.733000 & -3.747000 & -0.491000 & \mathrm{H} & 5.089000 & -6.014000 & 2.205000 \\ \mathrm{H} & -7.187000 & -5.052000 & -1.982000 & \mathrm{H} & 4.760000 & -5.397000 & -0.186000 \\ \mathrm{H} & -8.493000 & -3.918000 & -3.767000 & \mathrm{H} & 2.646000 & -4.383000 & -0.927000 \\ \mathrm{H} & -8.319000 & -1.454000 & -4.042000 & & & & \end{array}$

\section{with M06 functional}

$\begin{array}{lccccccc}\mathrm{Pd} & 1.770000 & 1.316000 & -0.341000 & \mathrm{C} & 0.675000 & 4.724000 & -4.401000 \\ \mathrm{Pd} & 0.254000 & -1.208000 & -0.002000 & \mathrm{C} & -1.428000 & 4.363000 & -3.062000 \\ \mathrm{Pd} & -1.192000 & 1.420000 & 0.056000 & \mathrm{C} & -0.401000 & 3.004000 & 1.005000 \\ \mathrm{P} & 0.005000 & -3.444000 & 0.695000 & \mathrm{C} & 0.888000 & 4.966000 & 2.144000 \\ \mathrm{P} & 2.293000 & -0.507000 & 0.937000 & \mathrm{C} & 2.141000 & 5.143000 & 1.291000 \\ \mathrm{P} & -1.840000 & -0.571000 & -0.844000 & \mathrm{C} & 1.242000 & 4.502000 & 3.554000 \\ \mathrm{Si} & 4.495000 & -1.352000 & -1.128000 & \mathrm{C} & 0.067000 & 6.251000 & 2.186000 \\ \mathrm{Si} & 4.117000 & 1.729000 & -0.485000 & \mathrm{C} & 4.788000 & 0.425000 & -1.694000 \\ \mathrm{Si} & -5.007000 & -0.634000 & -0.480000 & \mathrm{C} & 3.847000 & -1.441000 & 0.650000 \\ \mathrm{Si} & -3.491000 & 2.063000 & 0.190000 & \mathrm{C} & 4.463000 & 3.463000 & -1.182000 \\ \mathrm{~N} & 0.702000 & 3.573000 & -2.297000 & \mathrm{C} & 4.997000 & 4.480000 & -0.378000 \\ \mathrm{~N} & 0.087000 & 3.931000 & 1.536000 & \mathrm{C} & 5.152000 & 5.780000 & -0.854000 \\ \mathrm{C} & 1.119000 & 2.711000 & -1.619000 & \mathrm{C} & 4.791000 & 6.093000 & -2.160000 \\ \mathrm{C} & 0.067000 & 4.656000 & -3.004000 & \mathrm{C} & 4.284000 & 5.093000 & -2.987000 \\ \mathrm{C} & 0.345000 & 5.933000 & -2.216000 & \mathrm{C} & 4.117000 & 3.801000 & -2.499000\end{array}$




\begin{tabular}{|c|c|c|c|c|c|c|c|}
\hline $\mathrm{C}$ & 5.123000 & 1.591000 & 1.119000 & $\mathrm{C}$ & -5.756000 & -1.668000 & 2.109000 \\
\hline $\mathrm{C}$ & 4.561000 & 1.959000 & 2.350000 & $\mathrm{C}$ & -6.632000 & -1.892000 & 8000 \\
\hline $\mathrm{C}$ & 5.237000 & 1.772000 & 3.551000 & $\mathrm{C}$ & -7.906000 & -1.337000 & 3.146000 \\
\hline $\mathrm{C}$ & 6.505000 & 1.197000 & 3.547000 & $\mathrm{C}$ & -8.302000 & -0.561000 & 2.059000 \\
\hline $\mathrm{C}$ & 7.093000 & 0.833000 & 2.339000 & $\mathrm{C}$ & -7.424000 & -0.346000 & 1.004000 \\
\hline $\mathrm{C}$ & 6.411000 & 1.037000 & 1.141000 & $\mathrm{C}$ & -5.806000 & -1.566000 & -1.915000 \\
\hline $\mathrm{C}$ & 6.116000 & -2.318000 & -1.154000 & $\mathrm{C}$ & -5.871000 & -2.966000 & -1.885000 \\
\hline $\mathrm{C}$ & 6.904000 & -2.311000 & -2.314000 & $\mathrm{C}$ & -6.360000 & -3.691000 & -2.966000 \\
\hline $\mathrm{C}$ & 8.092000 & -3.030000 & -2.387000 & $\mathrm{C}$ & -6.818000 & -3.022000 & -4.099000 \\
\hline $\mathrm{C}$ & 8.518000 & -3.778000 & -1.293000 & $\mathrm{C}$ & -6.794000 & -1.631000 & -4.138000 \\
\hline $\mathrm{C}$ & 7.754000 & -3.799000 & -0.131000 & $\mathrm{C}$ & -6.291000 & -0.915000 & -3.054000 \\
\hline $\mathrm{C}$ & 6.567000 & -3.074000 & -0.064000 & $\mathrm{C}$ & -2.059000 & -0.553000 & -2.657000 \\
\hline $\mathrm{C}$ & 3.277000 & -2.229000 & -2.273000 & $\mathrm{C}$ & -1.324000 & 0.385000 & -3.401000 \\
\hline $\mathrm{C}$ & 3.495000 & -3.556000 & -2.667000 & $\mathrm{C}$ & -1.377000 & 0.390000 & -4.789000 \\
\hline $\mathrm{C}$ & 2.558000 & -4.252000 & -3.427000 & $\mathrm{C}$ & -2.160000 & -0.545000 & -5.466000 \\
\hline $\mathrm{C}$ & 1.377000 & -3.626000 & -3.815000 & $\mathrm{C}$ & -2.887000 & -1.482000 & -4.741000 \\
\hline $\mathrm{C}$ & 1.146000 & -2.300000 & -3.457000 & $\mathrm{C}$ & -2.835000 & -1.489000 & -3.348000 \\
\hline $\mathrm{C}$ & 2.088000 & -1.611000 & -2.697000 & $\mathrm{C}$ & -0.739000 & -4.699000 & -0.415000 \\
\hline $\mathrm{C}$ & -4.783000 & 1.175000 & -0.895000 & $\mathrm{C}$ & -0.756000 & -6.063000 & -0.103000 \\
\hline $\mathrm{C}$ & -3.315000 & -1.420000 & -0.134000 & $\mathrm{C}$ & -1.376000 & -6.968000 & -0.958000 \\
\hline $\mathrm{C}$ & 2.209000 & -0.285000 & 2.746000 & $\mathrm{C}$ & -1.984000 & -6.520000 & -2.129000 \\
\hline $\mathrm{C}$ & 1.261000 & 0.611000 & 3.264000 & $\mathrm{C}$ & -1.964000 & -5.167000 & -2.451000 \\
\hline $\mathrm{C}$ & 1.081000 & 0.744000 & 4.635000 & $\mathrm{C}$ & -1.335000 & -4.263000 & -1.599000 \\
\hline $\mathrm{C}$ & 1.838000 & -0.027000 & 5.518000 & $\mathrm{C}$ & -1.133000 & -3.389000 & 2.136000 \\
\hline $\mathrm{C}$ & 2.780000 & -0.919000 & 5.015000 & $\mathrm{C}$ & -0.873000 & -2.423000 & 3.119000 \\
\hline $\mathrm{C}$ & 2.970000 & -1.044000 & 3.641000 & $\mathrm{C}$ & -1.742000 & -2.249000 & 4.187000 \\
\hline $\mathrm{C}$ & -3.721000 & 3.913000 & -0.198000 & $\mathrm{C}$ & -2.903000 & -3.014000 & 4.278000 \\
\hline $\mathrm{C}$ & -3.138000 & 4.891000 & 0.625000 & $\mathrm{C}$ & -3.173000 & -3.970000 & 3.304000 \\
\hline $\mathrm{C}$ & -3.222000 & 6.245000 & 0.318000 & $\mathrm{C}$ & -2.293000 & -4.160000 & 2.240000 \\
\hline $\mathrm{C}$ & -3.905000 & 6.661000 & -0.823000 & $\mathrm{C}$ & 1.511000 & -4.295000 & 1.312000 \\
\hline $\mathrm{C}$ & -4.509000 & 5.714000 & -1.643000 & $\mathrm{C}$ & 1.863000 & -4.345000 & 2.663000 \\
\hline $\mathrm{C}$ & -4.413000 & 4.359000 & -1.331000 & $\mathrm{C}$ & 3.101000 & -4.855000 & 3.050000 \\
\hline $\mathrm{C}$ & -4.021000 & 1.796000 & 1.994000 & $\mathrm{C}$ & 3.997000 & -5.326000 & 2.097000 \\
\hline $\mathrm{C}$ & -3.232000 & 1.096000 & 2.917000 & $\mathrm{C}$ & 3.648000 & -5.293000 & 0.747000 \\
\hline $\mathrm{C}$ & -3.688000 & 0.838000 & 4.209000 & $\mathrm{C}$ & 2.419000 & -4.775000 & 0.357000 \\
\hline $\mathrm{C}$ & -4.945000 & 1.281000 & 4.604000 & $\mathrm{H}$ & -0.122000 & 6.786000 & -2.724000 \\
\hline $\mathrm{C}$ & -5.743000 & 1.988000 & 3.708000 & $\mathrm{H}$ & -0.087000 & 5.855000 & -1.209000 \\
\hline $\mathrm{C}$ & -5.282000 & 2.238000 & 2.420000 & $\mathrm{H}$ & 1.426000 & 6.111000 & -2.137000 \\
\hline $\mathrm{C}$ & -6.132000 & -0.890000 & 1.009000 & $\mathrm{H}$ & 0.212000 & 5.542000 & -4.96400 \\
\hline
\end{tabular}




\begin{tabular}{|c|c|c|c|c|c|c|c|}
\hline $\mathrm{H}$ & 1.755000 & 4.902000 & -4.343000 & $\mathrm{H}$ & -4.439000 & 1.255000 & -1.939000 \\
\hline & 0.502000 & 3.786000 & -4.942000 & $\mathrm{H}$ & -3.116000 & -1.387000 & 9000 \\
\hline & -1.943000 & 5.179000 & -3.584000 & $\mathrm{H}$ & -3.303000 & -2.486000 & -0.412000 \\
\hline & -1.627000 & 3.423000 & -3.591000 & $\mathrm{H}$ & 0.651000 & 1.193000 & 2.571000 \\
\hline & -1.847000 & 4.281000 & -2.051000 & $\mathrm{H}$ & 0.340000 & 1.446000 & 5.01400 \\
\hline & 2.783000 & 5.916000 & 1.731000 & $\mathrm{H}$ & 1.693000 & 0.069000 & 6.59200 \\
\hline & 2.707000 & 4.206000 & 1.233000 & $\mathrm{H}$ & 3.376000 & -1.524000 & 5.696000 \\
\hline & 1.890000 & 5.438000 & 0.264000 & $\mathrm{H}$ & 3.708000 & -1.749000 & 3.264000 \\
\hline & 1.862000 & 5.262000 & 4.043000 & $\mathrm{H}$ & -2.601000 & 4.580000 & 1.522000 \\
\hline & 0.334000 & 4.353000 & 4.151000 & $\mathrm{H}$ & -2.764000 & 6.982000 & 0.978000 \\
\hline & 1.798000 & 3.557000 & 3.529000 & $\mathrm{H}$ & -3.975000 & 7.720000 & -1.064000 \\
\hline & 0.655000 & 7.046000 & 2.658000 & $\mathrm{H}$ & -5.055000 & 6.029000 & -2.530000 \\
\hline & -0.205000 & 6.573000 & 1.174000 & $\mathrm{H}$ & -4.883000 & 3.634000 & -1.996000 \\
\hline & -0.854000 & 6.107000 & 2.764000 & $\mathrm{H}$ & -2.247000 & 0.729000 & 2.610000 \\
\hline & 4.274000 & 0.569000 & -2.654000 & $\mathrm{H}$ & -3.063000 & 0.279000 & 4.904000 \\
\hline & 5.862000 & 0.578000 & -1.878000 & $\mathrm{H}$ & -5.308000 & 1.071000 & 5.609000 \\
\hline $\mathrm{H}$ & 3.677000 & -2.500000 & 0.893000 & $\mathrm{H}$ & -6.732000 & 2.328000 & 4.009000 \\
\hline & 4.630000 & -1.052000 & 1.319000 & $\mathrm{H}$ & -5.923000 & 2.784000 & 1.725000 \\
\hline & 5.300000 & 4.252000 & 0.645000 & $\mathrm{H}$ & -4.762000 & -2.114000 & 2.149000 \\
\hline$t$ & 5.565000 & 6.549000 & -0.203000 & $\mathrm{H}$ & -6.313000 & -2.500000 & 4.014000 \\
\hline 11 & 4.920000 & 7.105000 & -2.539000 & $\mathrm{H}$ & -8.593000 & -1.509000 & 3.972000 \\
\hline 11 & 4.028000 & 5.322000 & -4.022000 & $\mathrm{H}$ & -9.300000 & -0.127000 & 2.034000 \\
\hline & 3.700000 & 3.040000 & -3.161000 & $\mathrm{H}$ & -7.753000 & 0.253000 & 0.151000 \\
\hline & 3.548000 & 2.365000 & 2.370000 & $\mathrm{H}$ & -5.519000 & -3.505000 & -1.001000 \\
\hline 11 & 4.761000 & 2.053000 & 4.489000 & $\mathrm{H}$ & -6.389000 & -4.778000 & -2.925000 \\
\hline & 7.033000 & 1.030000 & 4.484000 & $\mathrm{H}$ & -7.200000 & -3.585000 & -4.948000 \\
\hline & 8.084000 & 0.385000 & 2.327000 & $\mathrm{H}$ & -7.159000 & -1.104000 & -5.017000 \\
\hline 11 & 6.891000 & 0.738000 & 0.208000 & $\mathrm{H}$ & -6.257000 & 0.174000 & -3.106000 \\
\hline $\mathrm{H}$ & 6.578000 & -1.733000 & -3.181000 & $\mathrm{H}$ & -0.702000 & 1.110000 & -2.871000 \\
\hline U & 8.686000 & -3.010000 & -3.298000 & $\mathrm{H}$ & -0.801000 & 1.127000 & -5.347000 \\
\hline & 9.446000 & -4.343000 & -1.346000 & $\mathrm{H}$ & -2.202000 & -0.539000 & -6.553000 \\
\hline $\mathrm{H}$ & 8.083000 & -4.383000 & 0.727000 & $\mathrm{H}$ & -3.519000 & -2.208000 & -5.251000 \\
\hline 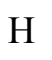 & 5.978000 & -3.109000 & 0.853000 & $\mathrm{H}$ & -3.423000 & -2.225000 & -2.804000 \\
\hline & 4.416000 & -4.059000 & -2.369000 & $\mathrm{H}$ & -0.283000 & -6.413000 & 0.814000 \\
\hline 11 & 2.750000 & -5.284000 & -3.716000 & $\mathrm{H}$ & -1.385000 & -8.027000 & -0.710000 \\
\hline 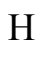 & 0.635000 & -4.167000 & -4.399000 & $\mathrm{H}$ & -2.469000 & -7.231000 & -2.794000 \\
\hline & 0.228000 & -1.799000 & -3.764000 & $\mathrm{H}$ & -2.425000 & -4.810000 & -3.370000 \\
\hline 11 & 1.885000 & -0.570000 & -2.427000 & $\mathrm{H}$ & -1.287000 & -3.203000 & -1.854000 \\
\hline & -5.749000 & 1.698000 & -0.819000 & $\mathrm{H}$ & 0.011000 & -1.789000 & 3.036000 \\
\hline
\end{tabular}




$\begin{array}{rrrrrrrr}\mathrm{H} & -1.516000 & -1.498000 & 4.943000 & \mathrm{H} & 3.362000 & -4.880000 & 4.106000 \\ \mathrm{H} & -3.599000 & -2.856000 & 5.099000 & \mathrm{H} & 4.965000 & -5.720000 & 2.400000 \\ \mathrm{H} & -4.082000 & -4.566000 & 3.363000 & \mathrm{H} & 4.345000 & -5.655000 & -0.007000 \\ \mathrm{H} & -2.523000 & -4.904000 & 1.479000 & \mathrm{H} & 2.174000 & -4.723000 & -0.706000 \\ \mathrm{H} & 1.173000 & -3.980000 & 3.421000 & & & & \end{array}$




\section{$\mathrm{X}$-ray data collection and reduction}

X-ray crystallography for compound 2 was performed on a Rigaku Saturn CCD area detector with graphite monochromated Mo-K $\alpha$ radiation $(\lambda=0.71075 \AA$ ). Single crystals of 3 suitable for X-ray crystallography were analyzed by synchrotron radiation at beam line BL02B1 ( $\lambda=0.41170 \AA$ ) of Spring-8 (Hyogo, Japan) using PILATUS3 X CdTe 1M detector. The data were collected at 113(2) K for 2 and 100(1) K for 3 using $\omega$ scan in the $\theta$ range of $1.78 \leq \theta \leq 31.07 \mathrm{deg}$ for 2 and $0.44 \leq \theta \leq 15.56 \mathrm{deg}$ for $\mathbf{3}$. The data obtained were processed using Crystal-Clear (Rigaku) on a Pentium computer, and were corrected for Lorentz and polarization effects. The structures were solved by direct methods ${ }^{10}$, and expanded using Fourier techniques. Hydrogen atoms were refined using the riding model. The final cycle of full-matrix least-squares refinement on $F^{2}$ was based on 5,752 observed reflections and 244 variable parameters for $\mathbf{2}$ and 18,588 observed reflections and 949 variable parameters for 3. Neutral atom scattering factors were taken from International Tables for Crystallography (IT), Vol. C, Table 6.1.1.4 ${ }^{11}$. Anomalous dispersion effects were included in Fcalc ${ }^{12}$; the values for $\Delta \mathrm{f}^{\prime}$ and $\Delta \mathrm{f}^{\prime \prime}$ were those of Creagh and McAuley ${ }^{13}$. The values for the mass attenuation coefficients are those of Creagh and Hubbell ${ }^{14}$. All calculations were performed using the CrystalStructure ${ }^{15}$ crystallographic software package except for refinement, which was performed using SHELXL Version 2017/1 ${ }^{16}$. Details of final refinement as well as the bond lengths and angle are summarized in Tables S3 and S4, and the numbering scheme employed is also shown in Figures S7 and S8, which were drawn with ORTEP at 50\% probability ellipsoids. CCDC 2091603 (2) and 2091604 (3) contains the supplementary crystallographic data for 2. 


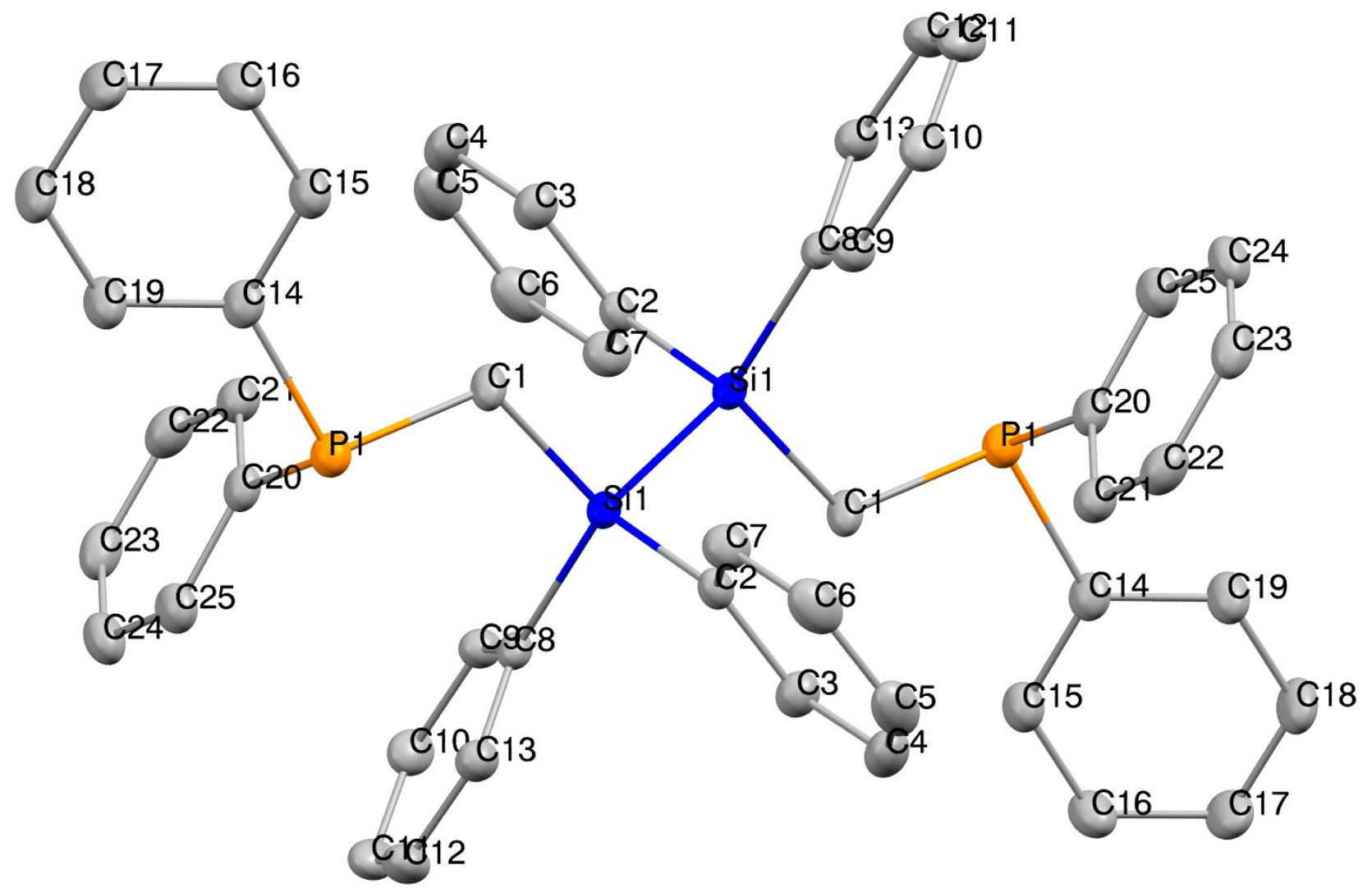

Figure S7. ORTEP drawing of 2 (50\% probability of the thermal ellipsoids). Hydrogen atoms were omitted for clarity. 
Table S3-1. Crystal data and structure refinement for 2.

Empirical Formula

Formula Weight

Crystal Color, Habit

Crystal Dimensions

Crystal System

Lattice Type

Lattice Parameters

Space Group

$\mathrm{Z}$ value

$\mathrm{D}_{\text {calc }}$

$\mathrm{F}_{000}$

$\mu(\operatorname{MoK} \alpha)$

Diffractometer

Radiation

Voltage, Current

Temperature

Detector Aperture

Data Images

$\omega$ oscillation Range $(\chi=45.0, \phi=0.0)$

Exposure Rate

Detector Swing Angle

$\omega$ oscillation Range $(\chi=45.0, \phi=90.0)$

Exposure Rate

Detector Swing Angle

$\omega$ oscillation Range $(\chi=45.0, \phi=180.0)$

Exposure Rate

Detector Swing Angle

Detector Position

Pixel Size

$2 \theta_{\max }$
$\mathrm{C}_{50} \mathrm{H}_{42} \mathrm{P}_{2} \mathrm{Si}_{2}$

761.00

colorless, bloak

$0.100 \times 0.100 \times 0.080 \mathrm{~mm}$

triclinic

Primitive

$\mathrm{a}=8.4807(4) \AA$

$\mathrm{b}=10.6727(6) \AA$

$\mathrm{c}=12.2112(6) \AA$

$\alpha=69.951(5)^{o}$

$\beta=79.994(4){ }^{\circ}$

$\gamma=78.697(5)^{\mathrm{o}}$

$\mathrm{V}=1011.31(10) \AA^{3}$

P-1 (\#2)

1

$1.249 \mathrm{~g} / \mathrm{cm}^{3}$

400.00

$2.017 \mathrm{~cm}^{-1}$

Saturn 724

$\operatorname{MoK} \alpha(\lambda=0.71073 \AA)$

graphite monochromated

$50 \mathrm{kV}, 24 \mathrm{~mA}$

$-150.0^{\circ} \mathrm{C}$

$72.8 \times 72.8 \mathrm{~mm}$

1080 exposures

$-70.0-110.0^{\circ}$

$10.0 \mathrm{sec} . / \mathrm{O}$

$20.00^{\circ}$

$-70.0-110.0^{\circ}$

$10.0 \mathrm{sec} . / \mathrm{O}$

$20.00^{\circ}$

$-70.0-110.0^{\circ}$

$10.0 \mathrm{sec} . / \mathrm{O}$

$20.00^{\circ}$

$45.00 \mathrm{~mm}$

$0.035 \mathrm{~mm}$

$61.9^{\circ}$ 
No. of Reflections Measured

Corrections

Structure Solution

Refinement

Function Minimized

Least Squares Weights

$2 \theta_{\text {max }}$ cutoff

Anomalous Dispersion

No. Observations (All reflections)

No. Variables

Reflection/Parameter Ratio

Residuals: R1 (I $>2.00 \sigma(\mathrm{I})$ )

Residuals: R (All reflections)

Residuals: wR2 (All reflections)

Goodness of Fit Indicator

Max Shift/Error in Final Cycle

Maximum peak in Final Diff. Map

Minimum peak in Final Diff. Map
Total: 13902

Unique: $5752\left(\mathrm{R}_{\text {int }}=0.0447\right)$

Lorentz-polarization

Absorption

(trans. factors: $0.506-0.984$ )

Direct Methods

Full-matrix least-squares on $\mathrm{F}^{2}$

$\Sigma \mathrm{w}\left(\mathrm{Fo}^{2}-\mathrm{Fc}^{2}\right)^{2}$

$\mathrm{w}=1 /\left[\sigma^{2}\left(\mathrm{Fo}^{2}\right)+(0.0789 \cdot \mathrm{P})^{2}\right.$

$+0.2255 \cdot \mathrm{P}]$

where $\mathrm{P}=\left(\operatorname{Max}\left(\mathrm{Fo}^{2}, 0\right)+2 \mathrm{Fc}^{2}\right) / 3$

$61.9^{\circ}$

All non-hydrogen atoms

5752

244

23.57

0.0507

0.0694

0.1444

1.059

0.000

$0.86 \mathrm{e}^{-/} / \AA^{3}$

$-0.42 \mathrm{e}^{-} / \AA^{3}$ 
Table S3-2. Atomic coordinates and $\mathrm{B}_{\mathrm{iso}} / \mathrm{B}_{\mathrm{eq}}$

\begin{tabular}{|c|c|c|c|c|}
\hline atom & $\mathrm{x}$ & $\mathrm{y}$ & $\mathrm{z}$ & $\mathrm{B}_{\mathrm{eq}}$ \\
\hline P1 & $0.65081(6)$ & $0.13043(4)$ & $0.22525(4)$ & $1.512(10)$ \\
\hline Sil & $0.46603(6)$ & $0.39147(4)$ & $0.05362(4)$ & $1.098(10)$ \\
\hline $\mathrm{C} 1$ & $0.6634(2)$ & $0.27488(18)$ & $0.08934(14)$ & $1.56(3)$ \\
\hline $\mathrm{C} 2$ & $0.3557(2)$ & $0.33181(16)$ & $-0.03664(14)$ & $1.28(3)$ \\
\hline $\mathrm{C} 3$ & $0.1971(2)$ & $0.39521(18)$ & $-0.05984(16)$ & $1.68(3)$ \\
\hline $\mathrm{C} 4$ & $0.1114(3)$ & $0.3609(2)$ & $-0.12977(17)$ & 2.09(3) \\
\hline $\mathrm{C} 5$ & $0.1820(3)$ & $0.2589(2)$ & $-0.17785(17)$ & $2.30(4)$ \\
\hline C6 & $0.3366(3)$ & $0.1926(2)$ & $-0.15556(17)$ & $2.26(3)$ \\
\hline $\mathrm{C} 7$ & $0.4223(2)$ & $0.22907(18)$ & $-0.08558(16)$ & $1.74(3)$ \\
\hline $\mathrm{C} 8$ & $0.3199(2)$ & $0.39776(17)$ & $0.18799(14)$ & $1.27(3)$ \\
\hline $\mathrm{C} 9$ & $0.3123(2)$ & $0.49551(18)$ & $0.24260(15)$ & $1.50(3)$ \\
\hline $\mathrm{C} 10$ & $0.2063(2)$ & $0.4946(2)$ & $0.34405(16)$ & $1.85(3)$ \\
\hline $\mathrm{C} 11$ & $0.1081(2)$ & $0.3943(2)$ & $0.39365(17)$ & $2.13(3)$ \\
\hline $\mathrm{C} 12$ & $0.1135(2)$ & $0.2965(2)$ & $0.34069(17)$ & $1.98(3)$ \\
\hline $\mathrm{C} 13$ & $0.2176(2)$ & $0.29909(18)$ & $0.23868(15)$ & $1.61(3)$ \\
\hline $\mathrm{C} 14$ & $0.8667(2)$ & $0.05646(17)$ & $0.23490(16)$ & $1.59(3)$ \\
\hline $\mathrm{C} 15$ & $0.9801(2)$ & $0.06336(19)$ & $0.13559(16)$ & $1.92(3)$ \\
\hline $\mathrm{C} 16$ & $1.1378(3)$ & $-0.0026(2)$ & $0.14586(17)$ & $2.30(3)$ \\
\hline $\mathrm{C} 17$ & $1.1873(3)$ & $-0.0767(2)$ & $0.25543(18)$ & $2.43(4)$ \\
\hline $\mathrm{C} 18$ & $1.0765(3)$ & $-0.0843(2)$ & $0.35500(17)$ & $2.45(4)$ \\
\hline C19 & $0.9179(3)$ & $-0.0195(2)$ & $0.34490(17)$ & $2.17(3)$ \\
\hline $\mathrm{C} 20$ & $0.6176(2)$ & $0.21319(17)$ & $0.33909(15)$ & $1.52(3)$ \\
\hline $\mathrm{C} 21$ & $0.7037(2)$ & $0.31380(19)$ & $0.33548(16)$ & $1.82(3)$ \\
\hline $\mathrm{C} 22$ & $0.6725(3)$ & $0.3716(2)$ & $0.42452(17)$ & $2.06(3)$ \\
\hline $\mathrm{C} 23$ & $0.5569(3)$ & $0.3271(2)$ & $0.51987(17)$ & $2.14(3)$ \\
\hline $\mathrm{C} 24$ & $0.4708(3)$ & $0.2280(2)$ & $0.52416(16)$ & $2.23(3)$ \\
\hline $\mathrm{C} 25$ & $0.4994(2)$ & $0.17236(18)$ & $0.43339(16)$ & $1.86(3)$ \\
\hline
\end{tabular}

$\mathrm{B}_{\mathrm{eq}}=8 / 3 \pi^{2}\left(\mathrm{U}_{11}\left(\mathrm{aa}^{*}\right)^{2}+\mathrm{U}_{22}\left(\mathrm{bb}^{*}\right)^{2}+\mathrm{U}_{33}\left(\mathrm{cc}^{*}\right)^{2}+2 \mathrm{U}_{12}(\mathrm{aa} * \mathrm{bb}) \cos \gamma+2 \mathrm{U}_{13}(\mathrm{aa} * \mathrm{cc} *) \cos \beta+\right.$ $\left.2 \mathrm{U}_{23}\left(\mathrm{bb}^{*} \mathrm{cc} *\right) \cos \alpha\right)$

Table S3-3. Anisotropic displacement parameters

$\begin{array}{lllllll}\text { atom } & \mathrm{U}_{11} & \mathrm{U}_{22} & \mathrm{U}_{33} & \mathrm{U}_{12} & \mathrm{U}_{13} & \mathrm{U}_{23} \\ \text { P1 } & 0.0207(3) & 0.0171(2) & 0.0190(2) & 0.00093(17) & -0.00298(17) & -0.00670(17) \\ \text { Si1 } & 0.0140(2) & 0.0152(2) & 0.0128(2) & 0.00129(17) & -0.00210(16) & -0.00653(16) \\ \text { C1 } & 0.0210(9) & 0.0203(8) & 0.0151(8) & 0.0053(7) & -0.0034(7) & -0.0060(6) \\ \text { C2 } & 0.0197(9) & 0.0158(8) & 0.0134(7) & -0.0023(6) & -0.0016(6) & -0.0052(6) \\ \text { C3 } & 0.0220(9) & 0.0209(8) & 0.0226(8) & -0.0013(7) & -0.0047(7) & -0.0092(7)\end{array}$




$\begin{array}{lllllll}\text { C4 } & 0.0255(10) & 0.0296(10) & 0.0257(9) & -0.0059(8) & -0.0084(8) & -0.0071(8) \\ \text { C5 } & 0.0391(12) & 0.0345(11) & 0.0212(9) & -0.0170(9) & -0.0047(8) & -0.0113(8) \\ \text { C6 } & 0.0397(12) & 0.0279(10) & 0.0243(9) & -0.0117(9) & 0.0022(8) & -0.0152(8) \\ \text { C7 } & 0.0235(10) & 0.0219(9) & 0.0230(9) & -0.0018(7) & -0.0001(7) & -0.0119(7) \\ \text { C8 } & 0.0141(8) & 0.0195(8) & 0.0142(7) & 0.0015(6) & -0.0026(6) & -0.0064(6) \\ \text { C9 } & 0.0173(9) & 0.0231(9) & 0.0186(8) & -0.0026(7) & -0.0006(7) & -0.0098(7) \\ \text { C10 } & 0.0225(10) & 0.0309(10) & 0.0205(8) & -0.0001(8) & -0.0011(7) & -0.0156(7) \\ \text { C11 } & 0.0193(10) & 0.0428(12) & 0.0201(9) & -0.0038(8) & 0.0024(7) & -0.0144(8) \\ \text { C12 } & 0.0203(10) & 0.0314(10) & 0.0231(9) & -0.0077(8) & 0.0008(7) & -0.0074(8) \\ \text { C13 } & 0.0190(9) & 0.0235(9) & 0.0203(8) & -0.0024(7) & -0.0024(7) & -0.0091(7) \\ \text { C14 } & 0.0235(9) & 0.0146(8) & 0.0215(8) & 0.0025(7) & -0.0046(7) & -0.0066(6) \\ \text { C15 } & 0.0263(10) & 0.0225(9) & 0.0203(9) & 0.0023(8) & -0.0033(7) & -0.0050(7) \\ \text { C16 } & 0.0247(10) & 0.0305(10) & 0.0238(9) & 0.0029(8) & 0.0029(8) & -0.0049(8) \\ \text { C17 } & 0.0222(10) & 0.0311(10) & 0.0298(10) & 0.0077(8) & -0.0032(8) & -0.0045(8) \\ \text { C18 } & 0.0295(11) & 0.0310(11) & 0.0235(10) & 0.0096(9) & -0.0066(8) & -0.0033(8) \\ \text { C19 } & 0.0277(11) & 0.0268(10) & 0.0215(9) & 0.0056(8) & -0.0027(8) & -0.0048(7) \\ \text { C20 } & 0.0210(9) & 0.0174(8) & 0.0182(8) & 0.0036(7) & -0.0057(7) & -0.0058(6) \\ \text { C21 } & 0.0205(10) & 0.0264(9) & 0.0212(9) & 0.0002(7) & -0.0034(7) & -0.0079(7) \\ \text { C22 } & 0.0257(10) & 0.0248(9) & 0.0314(10) & 0.0009(8) & -0.0103(8) & -0.0127(8) \\ \text { C23 } & 0.0302(11) & 0.0301(10) & 0.0224(9) & 0.0086(8) & -0.0089(8) & -0.0146(8) \\ \text { C24 } & 0.0297(11) & 0.0304(10) & 0.0180(9) & 0.0022(8) & -0.0006(8) & -0.0038(7) \\ \text { C25 } & 0.0266(10) & 0.0183(8) & 0.0218(9) & 0.0015(7) & -0.0036(7) & -0.0038(7)\end{array}$

The general temperature factor expression: $\exp \left(-2 \pi^{2}\left(a * 2 U_{11} h^{2}+b^{* 2} U_{22} k^{2}+c * 2 U_{33} 1^{2}+2 a * b * U_{12} h k+\right.\right.$ $\left.\left.2 a * c * U_{13} h l+2 b * c * U_{23} k l\right)\right)$

Table S3-4. Bond lengths $(\AA)$

$\begin{array}{llllll}\text { atom } & \text { atom } & \text { distance } & \text { atom } & \text { atom } & \text { distance } \\ \text { P1 } & \text { C1 } & 1.8415(15) & \text { P1 } & \text { C14 } & 1.8528(19) \\ \text { P1 } & \text { C20 } & 1.844(2) & \text { Si1 } & \text { Si1 } & 2.3653(6) \\ \text { Si1 } & \text { C1 } & 1.8986(18) & \text { Si1 } & \text { C2 } & 1.889(2) \\ \text { Si1 } & \text { C8 } & 1.8894(17) & \mathrm{C} 2 & \mathrm{C} 3 & 1.412(2) \\ \mathrm{C} 2 & \mathrm{C} 7 & 1.403(3) & \mathrm{C} 3 & \mathrm{C} 4 & 1.388(3) \\ \mathrm{C} 4 & \mathrm{C} 5 & 1.396(3) & \mathrm{C} 5 & \mathrm{C} 6 & 1.386(3) \\ \mathrm{C} 6 & \mathrm{C} 7 & 1.402(4) & \mathrm{C} 8 & \mathrm{C} 9 & 1.405(3) \\ \mathrm{C} 8 & \mathrm{C} 13 & 1.403(3) & \mathrm{C} 9 & \mathrm{C} 10 & 1.397(3) \\ \mathrm{C} 10 & \mathrm{C} 11 & 1.391(3) & \mathrm{C} 11 & \mathrm{C} 12 & 1.394(4) \\ \mathrm{C} 12 & \mathrm{C} 13 & 1.391(3) & \mathrm{C} 14 & \mathrm{C} 15 & 1.401(3) \\ \mathrm{C} 14 & \mathrm{C} 19 & 1.403(3) & \mathrm{C} 15 & \mathrm{C} 16 & 1.389(3) \\ \mathrm{C} 16 & \mathrm{C} 17 & 1.390(3) & \mathrm{C} 17 & \mathrm{C} 18 & 1.390(3)\end{array}$




$\begin{array}{llllll}\mathrm{C} 18 & \mathrm{C} 19 & 1.392(3) & \mathrm{C} 20 & \mathrm{C} 21 & 1.397(3) \\ \mathrm{C} 20 & \mathrm{C} 25 & 1.395(2) & \mathrm{C} 21 & \mathrm{C} 22 & 1.389(3) \\ \mathrm{C} 22 & \mathrm{C} 23 & 1.396(3) & \mathrm{C} 23 & \mathrm{C} 24 & 1.380(3) \\ \mathrm{C} 24 & \mathrm{C} 25 & 1.393(3) & & & \end{array}$

Symmetry Operators:

(1) $-\mathrm{X}+1,-\mathrm{Y}+1,-\mathrm{Z}$

Table S3-5. Bond angles $\left({ }^{\circ}\right)$

\begin{tabular}{|c|c|c|c|c|c|c|c|}
\hline atom & atom & atom & angle & atom & atom & atom & angle \\
\hline $\mathrm{C} 1$ & $\mathrm{P} 1$ & $\mathrm{C} 14$ & $101.76(8)$ & $\mathrm{C} 1$ & $\mathrm{P} 1$ & $\mathrm{C} 20$ & $101.95(8)$ \\
\hline $\mathrm{C} 14$ & $\mathrm{P} 1$ & $\mathrm{C} 20$ & 99.99(9) & $\mathrm{Si}^{1}$ & Sil & $\mathrm{C} 1$ & $106.86(6)$ \\
\hline $\mathrm{Si}^{1}$ & Sil & $\mathrm{C} 2$ & $110.36(5)$ & $\mathrm{Si}^{1}$ & Sil & $\mathrm{C} 8$ & $110.20(6)$ \\
\hline $\mathrm{C} 1$ & Sil & $\mathrm{C} 2$ & $110.42(9)$ & $\mathrm{C} 1$ & Sil & $\mathrm{C} 8$ & $113.41(7)$ \\
\hline $\mathrm{C} 2$ & Sil & $\mathrm{C} 8$ & $105.64(8)$ & $\mathrm{P} 1$ & $\mathrm{C} 1$ & Sil & $115.77(9)$ \\
\hline Sil & $\mathrm{C} 2$ & $\mathrm{C} 3$ & $119.03(15)$ & Sil & $\mathrm{C} 2$ & $\mathrm{C} 7$ & $124.31(14)$ \\
\hline $\mathrm{C} 3$ & $\mathrm{C} 2$ & $\mathrm{C} 7$ & $116.65(19)$ & $\mathrm{C} 2$ & $\mathrm{C} 3$ & $\mathrm{C} 4$ & $122.24(19)$ \\
\hline $\mathrm{C} 3$ & $\mathrm{C} 4$ & $\mathrm{C} 5$ & $119.51(19)$ & $\mathrm{C} 4$ & $\mathrm{C} 5$ & $\mathrm{C} 6$ & $120.0(2)$ \\
\hline $\mathrm{C} 5$ & $\mathrm{C} 6$ & $\mathrm{C} 7$ & $119.9(2)$ & $\mathrm{C} 2$ & $\mathrm{C} 7$ & C6 & $121.69(18)$ \\
\hline Sil & $\mathrm{C} 8$ & C9 & $122.59(13)$ & Sil & $\mathrm{C} 8$ & C13 & $119.51(15)$ \\
\hline C9 & $\mathrm{C} 8$ & $\mathrm{C} 13$ & $117.86(16)$ & $\mathrm{C} 8$ & C9 & $\mathrm{C} 10$ & $121.09(18)$ \\
\hline C9 & $\mathrm{C} 10$ & $\mathrm{C} 11$ & $119.9(2)$ & $\mathrm{C} 10$ & $\mathrm{C} 11$ & $\mathrm{C} 12$ & $119.94(18)$ \\
\hline $\mathrm{C} 11$ & $\mathrm{C} 12$ & $\mathrm{C} 13$ & 119.91(19) & $\mathrm{C} 8$ & $\mathrm{C} 13$ & $\mathrm{C} 12$ & $121.3(2)$ \\
\hline P1 & $\mathrm{C} 14$ & $\mathrm{C} 15$ & $122.71(14)$ & $\mathrm{P} 1$ & $\mathrm{C} 14$ & C19 & $119.39(14)$ \\
\hline C15 & $\mathrm{C} 14$ & $\mathrm{C} 19$ & $117.69(17)$ & $\mathrm{C} 14$ & $\mathrm{C} 15$ & $\mathrm{C} 16$ & $121.07(17)$ \\
\hline $\mathrm{C} 15$ & $\mathrm{C} 16$ & $\mathrm{C} 17$ & $120.66(18)$ & $\mathrm{C} 16$ & $\mathrm{C} 17$ & $\mathrm{C} 18$ & $119.06(19)$ \\
\hline C17 & $\mathrm{C} 18$ & $\mathrm{C} 19$ & $120.41(18)$ & $\mathrm{C} 14$ & $\mathrm{C} 19$ & $\mathrm{C} 18$ & $121.10(17)$ \\
\hline $\mathrm{P} 1$ & $\mathrm{C} 20$ & $\mathrm{C} 21$ & $123.61(13)$ & $\mathrm{P} 1$ & $\mathrm{C} 20$ & $\mathrm{C} 25$ & $117.58(16)$ \\
\hline $\mathrm{C} 21$ & $\mathrm{C} 20$ & $\mathrm{C} 25$ & $118.81(19)$ & $\mathrm{C} 20$ & $\mathrm{C} 21$ & $\mathrm{C} 22$ & $120.52(17)$ \\
\hline $\mathrm{C} 21$ & $\mathrm{C} 22$ & $\mathrm{C} 23$ & $119.9(2)$ & $\mathrm{C} 22$ & $\mathrm{C} 23$ & $\mathrm{C} 24$ & $120.0(2)$ \\
\hline $\mathrm{C} 23$ & $\mathrm{C} 24$ & $\mathrm{C} 25$ & $119.97(18)$ & $\mathrm{C} 20$ & $\mathrm{C} 25$ & $\mathrm{C} 24$ & $120.7(2)$ \\
\hline
\end{tabular}

Symmetry Operators:

(1) $-\mathrm{X}+1,-\mathrm{Y}+1,-\mathrm{Z}$

Table S3-6. Torsion Angles $\left({ }^{\circ}\right)$

(Those having bond angles $>160$ or $<20$ degrees are excluded.)

$\begin{array}{llllllllll}\text { atom1 } & \text { atom2 } & \text { atom3 } & \text { atom4 } & \text { angle } & \text { atom1 } & \text { atom2 } & \text { atom3 } & \text { atom4 } & \text { angle } \\ \text { C1 } & \text { P1 } & \text { C14 } & \text { C15 } & -30.97(17) & \text { C1 } & \text { P1 } & \text { C14 } & \text { C19 } & 154.48(14) \\ \text { C14 } & \text { P1 } & \text { C1 } & \text { Si1 } & -174.12(12) & \text { C1 } & \text { P1 } & \text { C20 } & \text { C21 } & -44.36(14) \\ \text { C1 } & \text { P1 } & \text { C20 } & \text { C25 } & 135.48(11) & \text { C20 } & \text { P1 } & \text { C1 } & \text { Si1 } & -71.11(13)\end{array}$




\begin{tabular}{|c|c|c|c|c|c|c|c|c|c|}
\hline $\mathrm{C} 14$ & $\mathrm{P} 1$ & $\mathrm{C} 20$ & $\mathrm{C} 21$ & $60.05(13)$ & $\mathrm{C} 14$ & $\mathrm{P} 1$ & $\mathrm{C} 20$ & $\mathrm{C} 25$ & $-120.12(11)$ \\
\hline $\mathrm{C} 20$ & $\mathrm{P} 1$ & $\mathrm{C} 14$ & $\mathrm{C} 15$ & $-135.53(15)$ & $\mathrm{C} 20$ & $\mathrm{P} 1$ & $\mathrm{C} 14$ & $\mathrm{C} 19$ & $49.93(15)$ \\
\hline $\operatorname{Si}^{1}$ & Sil & $\mathrm{C} 1$ & $\mathrm{P} 1$ & $150.87(9)$ & $\mathrm{C} 1$ & Si1 & $\mathrm{Si}^{1}$ & $\mathrm{C} 1^{1}$ & $180.00(7)$ \\
\hline $\mathrm{C} 1$ & Sil & $\mathrm{Si}^{1}$ & $\mathrm{C} 2^{1}$ & $-59.92(7)$ & $\mathrm{C} 1$ & Si1 & $\mathrm{Si} 1^{1}$ & $\mathrm{C} 8^{1}$ & $56.37(7)$ \\
\hline $\mathrm{Si}^{1}{ }^{1}$ & Si1 & $\mathrm{C} 2$ & $\mathrm{C} 3$ & $-69.89(10)$ & $\mathrm{Si} 1^{1}$ & Si1 & $\mathrm{C} 2$ & $\mathrm{C} 7$ & $108.61(10)$ \\
\hline $\mathrm{C} 2$ & Si1 & $\mathrm{Si}^{1}$ & $\mathrm{C} 1^{1}$ & $59.92(6)$ & $\mathrm{C} 2$ & Si1 & $\mathrm{Si}^{1}$ & $\mathrm{C} 2^{1}$ & $180.00(6)$ \\
\hline $\mathrm{C} 2$ & Sil & $\mathrm{Si}^{1}$ & $\mathrm{C} 8^{1}$ & $-63.71(6)$ & $\mathrm{Si}^{1}$ & Si1 & $\mathrm{C} 8$ & C9 & $-26.25(13)$ \\
\hline $\mathrm{Si}^{1}{ }^{1}$ & Sil & $\mathrm{C} 8$ & $\mathrm{C} 13$ & $155.90(9)$ & $\mathrm{C} 8$ & Si1 & $\mathrm{Si} 1^{1}$ & $\mathrm{C} 1^{1}$ & $-56.37(7)$ \\
\hline $\mathrm{C} 8$ & Sil & $\mathrm{Si}^{1}$ & $\mathrm{C} 2^{1}$ & $63.71(7)$ & $\mathrm{C} 8$ & Si1 & $\mathrm{Si} 1^{1}$ & $\mathrm{C} 8^{1}$ & $180.00(7)$ \\
\hline $\mathrm{C} 1$ & Si1 & $\mathrm{C} 2$ & $\mathrm{C} 3$ & $172.20(9)$ & $\mathrm{C} 1$ & Si1 & $\mathrm{C} 2$ & $\mathrm{C} 7$ & $-9.30(13)$ \\
\hline $\mathrm{C} 2$ & Sil & $\mathrm{C} 1$ & $\mathrm{P} 1$ & $-89.09(12)$ & $\mathrm{C} 1$ & Sil & $\mathrm{C} 8$ & C9 & $93.49(13)$ \\
\hline $\mathrm{C} 1$ & Si1 & $\mathrm{C} 8$ & $\mathrm{C} 13$ & $-84.36(14)$ & $\mathrm{C} 8$ & Si1 & $\mathrm{C} 1$ & $\mathrm{P} 1$ & $29.24(15)$ \\
\hline $\mathrm{C} 2$ & Sil & $\mathrm{C} 8$ & C9 & $-145.46(11)$ & $\mathrm{C} 2$ & Si1 & $\mathrm{C} 8$ & $\mathrm{C} 13$ & $36.70(12)$ \\
\hline $\mathrm{C} 8$ & Sil & $\mathrm{C} 2$ & $\mathrm{C} 3$ & $49.22(11)$ & $\mathrm{C} 8$ & Si1 & $\mathrm{C} 2$ & $\mathrm{C} 7$ & $-132.28(11)$ \\
\hline Sil & $\mathrm{C} 2$ & $\mathrm{C} 3$ & $\mathrm{C} 4$ & 177.07(10) & Sil & $\mathrm{C} 2$ & $\mathrm{C} 7$ & $\mathrm{C} 6$ & $-177.55(10)$ \\
\hline $\mathrm{C} 3$ & $\mathrm{C} 2$ & $\mathrm{C} 7$ & C6 & $1.0(2)$ & $\mathrm{C} 7$ & $\mathrm{C} 2$ & $\mathrm{C} 3$ & $\mathrm{C} 4$ & $-1.5(2)$ \\
\hline $\mathrm{C} 2$ & $\mathrm{C} 3$ & $\mathrm{C} 4$ & $\mathrm{C} 5$ & $1.1(2)$ & $\mathrm{C} 3$ & $\mathrm{C} 4$ & $\mathrm{C} 5$ & $\mathrm{C} 6$ & $0.0(3)$ \\
\hline $\mathrm{C} 4$ & $\mathrm{C} 5$ & $\mathrm{C} 6$ & $\mathrm{C} 7$ & $-0.6(3)$ & $\mathrm{C} 5$ & $\mathrm{C} 6$ & $\mathrm{C} 7$ & $\mathrm{C} 2$ & $0.0(3)$ \\
\hline Sil & $\mathrm{C} 8$ & C9 & $\mathrm{C} 10$ & $-177.94(10)$ & Sil & $\mathrm{C} 8$ & $\mathrm{C} 13$ & $\mathrm{C} 12$ & $177.05(10)$ \\
\hline $\mathrm{C} 9$ & $\mathrm{C} 8$ & $\mathrm{C} 13$ & $\mathrm{C} 12$ & $-0.9(2)$ & $\mathrm{C} 13$ & $\mathrm{C} 8$ & C9 & $\mathrm{C} 10$ & $-0.1(2)$ \\
\hline $\mathrm{C} 8$ & C9 & $\mathrm{C} 10$ & $\mathrm{C} 11$ & $1.2(3)$ & C9 & $\mathrm{C} 10$ & $\mathrm{C} 11$ & $\mathrm{C} 12$ & $-1.3(3)$ \\
\hline $\mathrm{C} 10$ & $\mathrm{C} 11$ & $\mathrm{C} 12$ & $\mathrm{C} 13$ & $0.4(3)$ & $\mathrm{C} 11$ & $\mathrm{C} 12$ & $\mathrm{C} 13$ & $\mathrm{C} 8$ & $0.8(3)$ \\
\hline P1 & $\mathrm{C} 14$ & $\mathrm{C} 15$ & $\mathrm{C} 16$ & $-174.71(14)$ & $\mathrm{P} 1$ & $\mathrm{C} 14$ & $\mathrm{C} 19$ & $\mathrm{C} 18$ & $175.56(14)$ \\
\hline $\mathrm{C} 15$ & $\mathrm{C} 14$ & $\mathrm{C} 19$ & $\mathrm{C} 18$ & $0.7(3)$ & $\mathrm{C} 19$ & $\mathrm{C} 14$ & $\mathrm{C} 15$ & $\mathrm{C} 16$ & $-0.1(3)$ \\
\hline $\mathrm{C} 14$ & $\mathrm{C} 15$ & $\mathrm{C} 16$ & $\mathrm{C} 17$ & $-0.4(3)$ & $\mathrm{C} 15$ & $\mathrm{C} 16$ & $\mathrm{C} 17$ & $\mathrm{C} 18$ & $0.2(4)$ \\
\hline $\mathrm{C} 16$ & $\mathrm{C} 17$ & $\mathrm{C} 18$ & $\mathrm{C} 19$ & $0.5(4)$ & $\mathrm{C} 17$ & $\mathrm{C} 18$ & C19 & $\mathrm{C} 14$ & $-0.9(4)$ \\
\hline P1 & $\mathrm{C} 20$ & $\mathrm{C} 21$ & $\mathrm{C} 22$ & $-179.87(10)$ & $\mathrm{P} 1$ & $\mathrm{C} 20$ & $\mathrm{C} 25$ & $\mathrm{C} 24$ & $178.32(10)$ \\
\hline $\mathrm{C} 21$ & $\mathrm{C} 20$ & $\mathrm{C} 25$ & $\mathrm{C} 24$ & $-1.8(2)$ & $\mathrm{C} 25$ & $\mathrm{C} 20$ & $\mathrm{C} 21$ & $\mathrm{C} 22$ & $0.3(2)$ \\
\hline $\mathrm{C} 20$ & $\mathrm{C} 21$ & $\mathrm{C} 22$ & $\mathrm{C} 23$ & $1.4(3)$ & $\mathrm{C} 21$ & $\mathrm{C} 22$ & $\mathrm{C} 23$ & $\mathrm{C} 24$ & $-1.6(3)$ \\
\hline $\mathrm{C} 22$ & $\mathrm{C} 23$ & $\mathrm{C} 24$ & $\mathrm{C} 25$ & $0.1(3)$ & $\mathrm{C} 23$ & $\mathrm{C} 24$ & $\mathrm{C} 25$ & $\mathrm{C} 20$ & $1.6(3)$ \\
\hline
\end{tabular}

(1) $-\mathrm{X}+1,-\mathrm{Y}+1,-\mathrm{Z}$ 


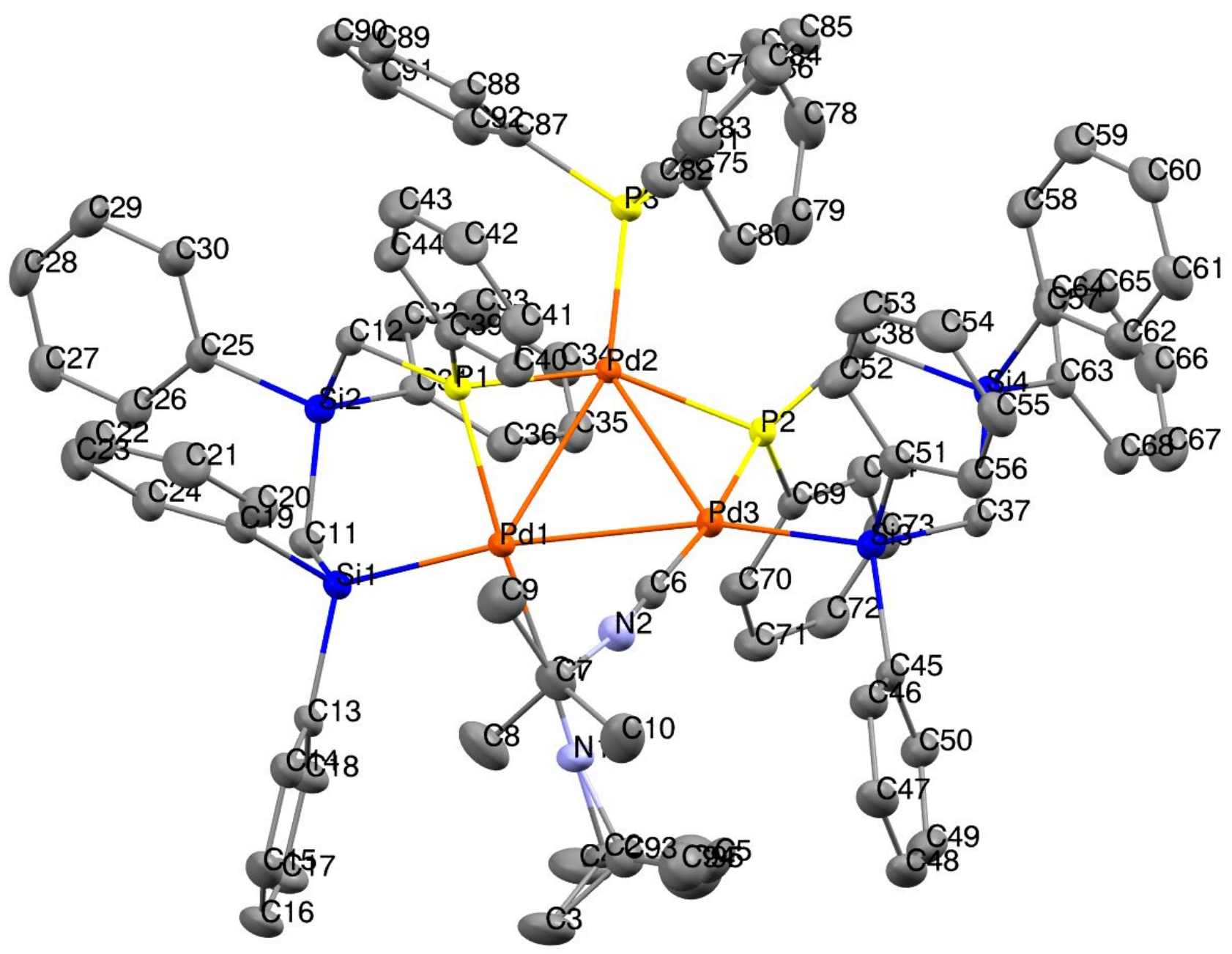

Figure S8. ORTEP drawing of $\mathbf{3}$ (50\% probability of the thermal ellipsoids). Hydrogen atoms were omitted for clarity. Hydrogen atoms were omitted for clarity. Three tBu group and one phenyl group were found to be disordered. The site occupancy factor for these carbons was defined as follows: 0.8 for $\mathrm{C} 2, \mathrm{C} 4$ and $\mathrm{C} 5,0.2$ for C93, C94 and C95, respectively. 
Table S4-1. Crystal data and structure refinement for 3.

Empirical Formula

Formula Weight

Crystal Color, Habit

Crystal Dimensions

Crystal System

Lattice Type

Lattice Parameters

Space Group

$\mathrm{Z}$ value

$\mathrm{D}_{\text {calc }}$

$\mathrm{F}_{000}$

Diffractometer

unknown Radiation $(\lambda=0.41170 \AA)$

Voltage, Current

Temperature

Detector Aperture

Data Images

$\omega$ oscillation Range $(\chi=45.0, \phi=0.0)$

Exposure Rate

Detector Swing Angle

$\omega$ oscillation Range $(\chi=45.0, \phi=90.0)$

Exposure Rate

Detector Swing Angle

$\omega$ oscillation Range $(\chi=45.0, \phi=180.0)$

Exposure Rate

Detector Swing Angle

Detector Position

Pixel Size

$2 \theta_{\max }$

No. of Reflections Measured

Corrections
$\mathrm{C}_{92} \mathrm{H}_{91} \mathrm{~N}_{2} \mathrm{P}_{3} \mathrm{Pd}_{3} \mathrm{Si}_{4}$

1749.21

red, block

0.080 X 0.060 X $0.050 \mathrm{~mm}$

monoclinic

Primitive

$\mathrm{a}=14.1324(5) \AA$

$\mathrm{b}=26.9320(10) \AA$

$\mathrm{c}=21.6683(8) \AA$

$\beta=99.005(7)^{\circ}$

$\mathrm{V}=8145.7(6) \AA^{3}$

$\mathrm{P} 21 / \mathrm{c}(\# 14)$

4

$1.426 \mathrm{~g} / \mathrm{cm}^{3}$

3584.00

R-AXIS IV

monochromated

$8 \mathrm{kV}, 100 \mathrm{~mA}$

$-173.0^{\circ} \mathrm{C}$

$300.0 \times 300.0 \mathrm{~mm}$

1080 exposures

$0.0-180.0^{\circ}$

$600.0 \mathrm{sec} . / \mathrm{O}$

$25.00^{\circ}$

$0.0-180.0^{\circ}$

$600.0 \mathrm{sec} . / \mathrm{O}$

$25.00^{\circ}$

$0.0-180.0^{\circ}$

$600.0 \mathrm{sec} . / \mathrm{o}$

$02500^{\circ}$

$130.00 \mathrm{~mm}$

$0.172 \mathrm{~mm}$

$31.0^{\circ}$

Total: 183193

Unique: $18588\left(\mathrm{R}_{\mathrm{int}}=0.0511\right)$

Lorentz-polarization 
Absorption

(trans. factors: 0.863 - 1.000)

Structure Solution

Refinement

Function Minimized

Least Squares Weights

$2 \theta_{\max }$ cutoff

Anomalous Dispersion

No. Observations (All reflections)

No. Variables

Reflection/Parameter Ratio

Residuals: R1 (I>2.00б(I))

Residuals: R (All reflections)

Residuals: wR2 (All reflections)

Goodness of Fit Indicator

Max Shift/Error in Final Cycle

Maximum peak in Final Diff. Map

Minimum peak in Final Diff. Map
Direct Methods (SHELXT Version 2014/5)

Full-matrix least-squares on $\mathrm{F}^{2}$

$\Sigma \mathrm{W}\left(\mathrm{Fo}^{2}-\mathrm{Fc}^{2}\right)^{2}$

$\mathrm{W}=1 /\left[\sigma^{2}\left(\mathrm{Fo}^{2}\right)+(0.0279 \cdot \mathrm{P})^{2}\right.$

$+5.7288 \cdot \mathrm{P}]$

where $\mathrm{P}=\left(\operatorname{Max}\left(\mathrm{Fo}^{2}, 0\right)+2 \mathrm{Fc}^{2}\right) / 3$

$31.0^{\circ}$

All non-hydrogen atoms

18588

949

19.59

0.0224

0.0260

0.0568

0.967

0.006

$0.65 \mathrm{e}^{-/} / \AA^{3}$

$-0.50 \mathrm{e}^{-} / \AA^{3}$ 
Table S4-2. Atomic coordinates and $\mathrm{B}_{\mathrm{iso}} / \mathrm{B}_{\mathrm{eq}}$

\begin{tabular}{|c|c|c|c|c|c|}
\hline atom & $\mathrm{x}$ & $\mathrm{y}$ & $\mathrm{z}$ & $\mathrm{B}_{\mathrm{eq}}$ & \\
\hline Pd1 & $0.33689(2)$ & $0.69723(2)$ & $0.24041(2)$ & $1.262(2)$ & 1 \\
\hline $\mathrm{Pd} 2$ & $0.36023(2)$ & $0.59107(2)$ & $0.22261(2)$ & $1.183(2)$ & 1 \\
\hline $\mathrm{Pd} 3$ & $0.21308(2)$ & $0.62567(2)$ & $0.29151(2)$ & $1.327(2)$ & 1 \\
\hline $\mathrm{P} 1$ & $0.47096(3)$ & $0.65225(2)$ & $0.25037(2)$ & $1.278(6)$ & 1 \\
\hline $\mathrm{P} 2$ & $0.20825(3)$ & $0.56320(2)$ & $0.22436(2)$ & $1.341(6)$ & 1 \\
\hline P3 & $0.44384(3)$ & $0.52005(2)$ & $0.20992(2)$ & $1.468(6)$ & 1 \\
\hline Sil & $0.42208(3)$ & $0.76365(2)$ & $0.20708(2)$ & $1.468(7)$ & 1 \\
\hline $\mathrm{Si} 2$ & $0.51602(3)$ & $0.68899(2)$ & $0.12190(2)$ & $1.521(7)$ & 1 \\
\hline $\mathrm{Si} 3$ & $0.09568(3)$ & $0.58672(2)$ & $0.33890(2)$ & $1.483(7)$ & 1 \\
\hline $\mathrm{Si} 4$ & $0.08462(3)$ & $0.47936(2)$ & $0.27807(2)$ & $1.487(7)$ & 1 \\
\hline N1 & $0.13913(11)$ & $0.75084(6)$ & $0.20957(8)$ & $2.26(3)$ & 1 \\
\hline $\mathrm{N} 2$ & $0.24542(11)$ & $0.71385(6)$ & $0.38696(7)$ & $1.97(2)$ & 1 \\
\hline $\mathrm{C} 1$ & $0.21039(12)$ & $0.73005(6)$ & $0.22141(8)$ & $1.70(3)$ & 1 \\
\hline $\mathrm{C} 2$ & $0.04858(16)$ & $0.77573(9)$ & $0.18753(15)$ & $2.47(4)$ & 0.800000 \\
\hline $\mathrm{C} 3$ & $0.05504(18)$ & $0.82794(9)$ & $0.21397(13)$ & $3.70(5)$ & 1 \\
\hline $\mathrm{C} 4$ & $0.0360(2)$ & $0.77818(11)$ & $0.11597(14)$ & $3.89(6)$ & 0.800000 \\
\hline $\mathrm{C} 5$ & $-0.0296(2)$ & $0.74341(15)$ & $0.2080(3)$ & $5.20(9)$ & 0.800000 \\
\hline C6 & $0.23402(12)$ & $0.68133(6)$ & $0.35191(8)$ & $1.75(3)$ & 1 \\
\hline $\mathrm{C} 7$ & $0.27304(14)$ & $0.75780(7)$ & $0.42440(9)$ & $2.24(3)$ & 1 \\
\hline $\mathrm{C} 8$ & $0.2711(2)$ & $0.80118(8)$ & $0.37959(12)$ & $3.78(5)$ & 1 \\
\hline $\mathrm{C} 9$ & $0.37486(16)$ & $0.74922(9)$ & $0.45757(11)$ & $3.37(4)$ & 1 \\
\hline $\mathrm{C} 10$ & $0.20376(17)$ & $0.76446(9)$ & $0.47042(11)$ & $3.28(4)$ & 1 \\
\hline $\mathrm{C} 11$ & $0.44400(13)$ & $0.74644(6)$ & $0.12611(8)$ & $1.79(3)$ & 1 \\
\hline $\mathrm{C} 12$ & $0.56290(11)$ & $0.66353(6)$ & $0.20171(8)$ & $1.52(2)$ & 1 \\
\hline $\mathrm{C} 13$ & $0.35524(12)$ & $0.82487(6)$ & $0.19721(9)$ & $1.81(3)$ & 1 \\
\hline $\mathrm{C} 14$ & $0.35714(13)$ & $0.85842(6)$ & $0.24647(10)$ & $2.16(3)$ & 1 \\
\hline $\mathrm{C} 15$ & $0.30943(15)$ & $0.90361(7)$ & $0.23886(11)$ & $2.76(4)$ & 1 \\
\hline $\mathrm{C} 16$ & $0.25952(16)$ & $0.91667(7)$ & $0.18140(12)$ & $3.27(4)$ & 1 \\
\hline $\mathrm{C} 17$ & $0.25590(17)$ & $0.88450(8)$ & $0.13171(12)$ & $3.40(4)$ & 1 \\
\hline $\mathrm{C} 18$ & $0.30310(15)$ & $0.83892(7)$ & $0.13977(10)$ & $2.65(3)$ & 1 \\
\hline C19 & $0.54411(12)$ & $0.78022(6)$ & $0.25180(9)$ & $1.86(3)$ & 1 \\
\hline $\mathrm{C} 20$ & $0.56565(15)$ & $0.77537(7)$ & $0.31596(9)$ & $2.49(3)$ & 1 \\
\hline $\mathrm{C} 21$ & $0.65582(17)$ & $0.78765(9)$ & $0.34806(11)$ & $3.34(4)$ & 1 \\
\hline $\mathrm{C} 22$ & $0.72623(16)$ & $0.80480(8)$ & $0.31610(12)$ & $3.44(4)$ & 1 \\
\hline $\mathrm{C} 23$ & $0.70815(15)$ & $0.80882(8)$ & $0.25279(12)$ & $3.27(4)$ & 1 \\
\hline $\mathrm{C} 24$ & $0.61834(14)$ & $0.79668(7)$ & $0.22082(10)$ & $2.55(3)$ & 1 \\
\hline $\mathrm{C} 25$ & $0.62499(13)$ & $0.70252(6)$ & $0.08478(8)$ & $1.84(3)$ & 1 \\
\hline
\end{tabular}




\begin{tabular}{|c|c|c|c|c|}
\hline $\mathrm{C} 26$ & $0.62624(14)$ & $0.74004(7)$ & $0.04012(9)$ & $2.33(3)$ \\
\hline $\mathrm{C} 27$ & $0.70780(15)$ & $0.75001(8)$ & $0.01398(9)$ & $2.63(3)$ \\
\hline $\mathrm{C} 28$ & $0.78956(14)$ & $0.72231(8)$ & $0.03154(10)$ & $2.71(3)$ \\
\hline $\mathrm{C} 29$ & $0.79043(14)$ & $0.68543(8)$ & $0.07522(10)$ & $2.74(3)$ \\
\hline $\mathrm{C} 30$ & $0.70926(14)$ & $0.67580(7)$ & $0.10172(9)$ & $2.32(3)$ \\
\hline $\mathrm{C} 31$ & $0.43939(13)$ & $0.64187(6)$ & $0.07316(8)$ & $1.76(3)$ \\
\hline $\mathrm{C} 32$ & $0.47477(14)$ & $0.61361(7)$ & $0.02827(9)$ & $2.36(3)$ \\
\hline $\mathrm{C} 33$ & $0.41762(16)$ & $0.57923(8)$ & $-0.00842(10)$ & $2.83(3)$ \\
\hline $\mathrm{C} 34$ & $0.32356(15)$ & $0.57278(8)$ & $-0.00082(9)$ & $2.67(3)$ \\
\hline $\mathrm{C} 35$ & $0.28594(14)$ & $0.60065(7)$ & $0.04336(9)$ & $2.32(3)$ \\
\hline $\mathrm{C} 36$ & $0.34331(13)$ & $0.63456(7)$ & $0.07994(8)$ & $1.98(3)$ \\
\hline $\mathrm{C} 37$ & $0.01790(12)$ & $0.53551(6)$ & $0.29675(8)$ & $1.71(3)$ \\
\hline $\mathrm{C} 38$ & $0.19908(12)$ & $0.49926(6)$ & $0.25124(8)$ & $1.59(2)$ \\
\hline C39 & $0.53519(12)$ & $0.64546(6)$ & $0.32920(8)$ & $1.61(3)$ \\
\hline $\mathrm{C} 40$ & $0.48211(13)$ & $0.64441(7)$ & $0.37840(8)$ & $2.03(3)$ \\
\hline $\mathrm{C} 41$ & $0.52520(15)$ & $0.63309(8)$ & $0.43860(9)$ & $2.54(3)$ \\
\hline $\mathrm{C} 42$ & $0.62182(15)$ & $0.62231(8)$ & $0.45101(9)$ & $2.74(3)$ \\
\hline $\mathrm{C} 43$ & $0.67592(14)$ & $0.62414(8)$ & $0.40310(10)$ & $2.64(3)$ \\
\hline $\mathrm{C} 44$ & $0.63312(13)$ & $0.63583(7)$ & $0.34260(9)$ & $2.08(3)$ \\
\hline $\mathrm{C} 45$ & $0.00771(12)$ & $0.63424(6)$ & $0.36066(9)$ & $1.85(3)$ \\
\hline $\mathrm{C} 46$ & $0.02571(14)$ & $0.65999(7)$ & $0.41703(9)$ & $2.25(3)$ \\
\hline $\mathrm{C} 47$ & $-0.03566(16)$ & $0.69682(7)$ & $0.43226(10)$ & $2.71(3)$ \\
\hline $\mathrm{C} 48$ & $-0.11595(16)$ & $0.70897(8)$ & $0.39062(11)$ & $2.94(4)$ \\
\hline C49 & $-0.13523(15)$ & $0.68463(8)$ & $0.33379(11)$ & $3.00(4)$ \\
\hline C50 & $-0.07463(14)$ & $0.64744(7)$ & $0.31942(10)$ & $2.42(3)$ \\
\hline C51 & $0.15447(12)$ & $0.55719(6)$ & $0.41430(8)$ & $1.76(3)$ \\
\hline C52 & $0.25107(14)$ & $0.54454(8)$ & $0.42383(9)$ & $2.68(3)$ \\
\hline C53 & $0.29253(17)$ & $0.51870(10)$ & $0.47683(11)$ & $3.62(4)$ \\
\hline C54 & $0.23827(17)$ & $0.50604(8)$ & $0.52218(10)$ & $3.17(4)$ \\
\hline $\mathrm{C} 55$ & $0.14274(16)$ & $0.51852(7)$ & $0.51415(9)$ & $2.67(3)$ \\
\hline C56 & $0.10134(14)$ & $0.54338(7)$ & $0.46080(9)$ & $2.24(3)$ \\
\hline $\mathrm{C} 57$ & $0.11468(12)$ & $0.43648(6)$ & $0.34630(8)$ & $1.73(3)$ \\
\hline $\mathrm{C} 58$ & $0.19582(13)$ & $0.40632(7)$ & $0.35206(9)$ & $2.29(3)$ \\
\hline C59 & $0.21407(15)$ & $0.37094(8)$ & $0.39898(10)$ & $2.68(3)$ \\
\hline C60 & $0.15247(16)$ & $0.36602(8)$ & $0.44211(9)$ & $2.70(3)$ \\
\hline C61 & $0.07190(17)$ & $0.39543(8)$ & $0.43768(10)$ & $2.79(4)$ \\
\hline C62 & $0.05298(15)$ & $0.42981(7)$ & $0.38964(9)$ & $2.41(3)$ \\
\hline C63 & $0.01559(12)$ & $0.44121(6)$ & $0.21364(8)$ & $1.75(3)$ \\
\hline C64 & $0.06290(14)$ & $0.40690(6)$ & $0.18057(9)$ & $2.11(3)$ \\
\hline
\end{tabular}




\begin{tabular}{|c|c|c|c|c|c|}
\hline C65 & $0.01507(16)$ & $0.38159(7)$ & $0.12932(10)$ & $2.66(3)$ & 1 \\
\hline C66 & $-0.08170(16)$ & $0.38981(8)$ & $0.11016(10)$ & $2.96(4)$ & 1 \\
\hline C67 & $-0.13039(15)$ & $0.42178(8)$ & $0.14336(10)$ & $2.83(3)$ & 1 \\
\hline C68 & $-0.08224(13)$ & $0.44725(7)$ & $0.19451(9)$ & $2.20(3)$ & 1 \\
\hline C69 & $0.11781(12)$ & $0.57110(6)$ & $0.15560(8)$ & $1.59(2)$ & 1 \\
\hline $\mathrm{C} 70$ & $0.09708(13)$ & $0.61933(7)$ & $0.13360(9)$ & $2.11(3)$ & 1 \\
\hline C71 & $0.03226(14)$ & $0.62773(7)$ & $0.08004(10)$ & $2.57(3)$ & 1 \\
\hline $\mathrm{C} 72$ & $-0.01544(14)$ & $0.58858(8)$ & $0.04732(9)$ & $2.62(3)$ & 1 \\
\hline $\mathrm{C} 73$ & $0.00297(15)$ & $0.54092(8)$ & $0.06893(9)$ & $2.56(3)$ & 1 \\
\hline $\mathrm{C} 74$ & $0.07055(13)$ & $0.53204(7)$ & $0.12194(8)$ & $2.06(3)$ & 1 \\
\hline $\mathrm{C} 75$ & $0.39501(13)$ & $0.47452(6)$ & $0.15096(8)$ & $1.87(3)$ & 1 \\
\hline $\mathrm{C} 76$ & $0.44405(15)$ & $0.43100(7)$ & $0.14080(10)$ & $2.49(3)$ & 1 \\
\hline C77 & $0.40351(18)$ & $0.39701(8)$ & $0.09661(11)$ & $3.11(4)$ & 1 \\
\hline $\mathrm{C} 78$ & $0.31477(18)$ & $0.40580(8)$ & $0.06184(11)$ & $3.23(4)$ & 1 \\
\hline $\mathrm{C} 79$ & $0.26636(16)$ & $0.44905(8)$ & $0.07069(10)$ & $2.87(4)$ & 1 \\
\hline $\mathrm{C} 80$ & $0.30664(14)$ & $0.48338(7)$ & $0.11521(9)$ & $2.19(3)$ & 1 \\
\hline C81 & $0.45916(12)$ & $0.48684(6)$ & $0.28439(8)$ & $1.76(3)$ & 1 \\
\hline C82 & $0.47919(14)$ & $0.51588(7)$ & $0.33794(9)$ & $2.23(3)$ & 1 \\
\hline $\mathrm{C} 83$ & $0.49661(16)$ & $0.49471(8)$ & $0.39669(10)$ & $2.77(3)$ & 1 \\
\hline C84 & $0.49179(16)$ & $0.44372(8)$ & $0.40283(10)$ & $3.02(4)$ & 1 \\
\hline $\mathrm{C} 85$ & $0.46930(16)$ & $0.41467(7)$ & $0.35067(11)$ & $2.98(4)$ & 1 \\
\hline C86 & $0.45286(14)$ & $0.43554(7)$ & $0.29137(10)$ & $2.35(3)$ & 1 \\
\hline $\mathrm{C} 87$ & $0.56693(12)$ & $0.52654(6)$ & $0.19430(9)$ & $1.77(3)$ & 1 \\
\hline $\mathrm{C} 88$ & $0.64336(13)$ & $0.53363(7)$ & $0.24213(10)$ & $2.15(3)$ & 1 \\
\hline C89 & $0.73467(14)$ & $0.54399(7)$ & $0.22861(11)$ & $2.71(3)$ & 1 \\
\hline C90 & $0.74995(15)$ & $0.54690(8)$ & $0.16719(12)$ & $3.05(4)$ & 1 \\
\hline C91 & $0.67413(16)$ & $0.53969(7)$ & $0.11933(11)$ & $2.92(4)$ & 1 \\
\hline C92 & $0.58324(14)$ & $0.52993(7)$ & $0.13287(9)$ & $2.29(3)$ & 1 \\
\hline C93 & $0.0435(8)$ & $0.7748(4)$ & $0.2201(6)$ & $2.43(19)$ & 0.200000 \\
\hline C94 & $0.0162(9)$ & $0.7678(5)$ & $0.2834(6)$ & $3.7(2)$ & 0.200000 \\
\hline C95 & $-0.0267(17)$ & $0.7495(9)$ & $0.1742(11)$ & $6.6(5)$ & 0.200000 \\
\hline
\end{tabular}

$B_{\text {eq }}=8 / 3 \pi^{2}\left(U_{11}\left(a a^{*}\right)^{2}+U_{22}\left(b^{*}\right)^{2}+U_{33}\left(c c^{*}\right)^{2}+2 U_{12}\left(a a^{*} b^{*}\right) \cos \gamma+2 U_{13}\left(a a^{*} c c^{*}\right) \cos \beta+\right.$ $\left.2 \mathrm{U}_{23}\left(\mathrm{bb}^{*} \mathrm{cc} *\right) \cos \alpha\right)$

Table S4-3. Anisotropic displacement parameters

$\begin{array}{lllllll}\text { atom } & \mathrm{U}_{11} & \mathrm{U}_{22} & \mathrm{U}_{33} & \mathrm{U}_{12} & \mathrm{U}_{13} & \mathrm{U}_{23} \\ \mathrm{Pd} 1 & 0.01460(5) & 0.01342(5) & 0.01983(6) & 0.00074(4) & 0.00234(4) & 0.00098(4) \\ \mathrm{Pd} 2 & 0.01429(5) & 0.01280(5) & 0.01791(6) & 0.00021(4) & 0.00269(4) & -0.00025(4)\end{array}$




\begin{tabular}{|c|c|c|c|c|c|c|}
\hline $\mathrm{Pd} 3$ & $5(6)$ & $0.01559(6)$ & $0.01868(6)$ & $-0.00175(4)$ & 0.0 & (4) \\
\hline P1 & $0.01454(18)$ & $0.01442(17)$ & $0.01932(19)$ & $-0.00003(14)$ & $0.00177(15)$ & $0.00119(14)$ \\
\hline $\mathrm{P} 2$ & $.01619(18)$ & $0.01573(18)$ & $0.01926(19)$ & $-0.00179(14)$ & $0.00345(15)$ & $-0.00215(15$ \\
\hline 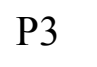 & $.01946(19)$ & $0.01455(18)$ & $.0221(2)$ & $0.00203(15)$ & $0.00445(16)$ & $-0.00029(15$ \\
\hline NII & $0.0183(2)$ & $0.01456(19)$ & $0.0229(2)$ & $-0.00021(16)$ & $0.00328(17)$ & $0.00128(16$ \\
\hline $\mathrm{Si} 2$ & $0.0187(2)$ & $0.0185(2)$ & $0.0211(2)$ & $0.00116(16)$ & $0.00455(17)$ & $0.00179(17$ \\
\hline & (2) & $0.0179(2)$ & $0.0223(2)$ & $-0.00047(16)$ & $0.00534(17)$ & $-0.00204(17$ \\
\hline $\mathrm{Si} 4$ & $.0176(2)$ & $0.0169(2)$ & $0.0222(2)$ & $-0.00262(16)$ & $0.00386(17)$ & -0.0 \\
\hline N1 & $0.0215(7)$ & $0.0221(7)$ & $0.0412(9)$ & $0.0046(6)$ & $0.0017(7)$ & $0.0006(7)$ \\
\hline $\mathrm{N} 2$ & $0.0289(8)$ & $0.0219(7)$ & $0.0239(7)$ & $-0.0006(6)$ & $0.0031(6)$ & $-0.0041(6)$ \\
\hline $\mathrm{C} 1$ & $.0219(8)$ & $0.0183(7)$ & $.0246(8)$ & $-0.0010(6)$ & $0.0037(6)$ & $0.0002(6)$ \\
\hline$c$ & $0.0182(11)$ & $0.0267(12)$ & $0.0473(17)$ & $0.0101(9)$ & $-0.0006(11)$ & $0.0032(11)$ \\
\hline $\mathrm{C} 3$ & $0.0517(14)$ & $0.0318(11)$ & $0.0561(15)$ & $0.0190(10)$ & $0.0054(11)$ & $-0.0064(10)$ \\
\hline 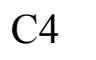 & $0.0547(18)$ & $0.0405(15)$ & $0.0444(16)$ & $0.0201(13)$ & $-0.0180(14)$ & $-0.0094(13)$ \\
\hline $\mathrm{C} 5$ & $0.0234(14)$ & $0.058(2)$ & $0.120(4)$ & $0.0013(13)$ & $0.0217(19)$ & $0.019(2)$ \\
\hline C6 & $0.0218(8)$ & $0.0215(8)$ & $0.0236(8)$ & $-0.0016(6)$ & $0.0045(6)$ & $0.0004(7)$ \\
\hline $\mathrm{C} 7$ & $0.0368(10)$ & $0.0221(8)$ & $0.0259(9)$ & $-0.0034(7)$ & $0.0040(8)$ & $-0.0091(7)$ \\
\hline o & $0.0737(17)$ & $0.0241(10)$ & $0.0459(13)$ & $-0.0020(10)$ & $0.0102(12)$ & $-0.0010(9)$ \\
\hline C9 & $0.0376(11)$ & $0.0480(13)$ & $0.0399(12)$ & $-0.0024(10)$ & $-0.0023(9)$ & $-0.0186(10)$ \\
\hline $\mathrm{C} 10$ & $0.0458(12)$ & $0.0408(12)$ & $0.0411(12)$ & $-0.0066(10)$ & $0.0165(10)$ & $-0.0194(10)$ \\
\hline 11 & $0.0244(8)$ & $0.0188(8)$ & $0.0251(8)$ & $0.0027(6)$ & $0.0048(7)$ & $0.0035(6)$ \\
\hline $\mathrm{C} 12$ & $0.0169(7)$ & $0.0180(7)$ & $0.0231(8)$ & $0.0000(6)$ & $0.0041(6)$ & $0.0012(6)$ \\
\hline $\mathrm{C} 13$ & $0.0225(8)$ & $0.0149(7)$ & $0.0322(9)$ & $-0.0015(6)$ & $0.0062(7)$ & $0.0022(6)$ \\
\hline $\mathrm{C} 14$ & $0.0273(9)$ & $0.0201(8)$ & $0.0359(10)$ & $-0.0019(7)$ & $0.0086(8)$ & $-0.0006(7)$ \\
\hline $\mathrm{C} 15$ & $0.0340(10)$ & $0.0195(9)$ & $0.0533(13)$ & -0.00 & 0.0 & -0. \\
\hline $\mathrm{C} 16$ & $0.0382(11)$ & $0.0187(9)$ & $0.0668(15)$ & $0.0080(8)$ & $0.0068(11)$ & $0.0023(9)$ \\
\hline $\mathrm{C} 17$ & $0.0469(13)$ & $0.0283(10)$ & $0.0509(14)$ & $0.0113(9)$ & $-0.0026(10)$ & $0.0065(9)$ \\
\hline C18 & $0.0386(11)$ & $0.0224(9)$ & $0.0377(11)$ & $0.0061(8)$ & $-0.0003(9)$ & $0.0006(8)$ \\
\hline C19 & $0.0233(8)$ & $0.0147(7)$ & $0.0319(9)$ & $-0.0010(6)$ & $0.0023(7)$ & $0.0014(6)$ \\
\hline $\mathrm{C} 20$ & $0.0327(10)$ & $0.0292(9)$ & $0.0314(10)$ & $-0.0015(8)$ & $0.0011(8)$ & $0.0012(8)$ \\
\hline $\mathrm{C} 21$ & $0.0433(12)$ & $0.0400(12)$ & $0.0382(12)$ & $-0.0019(10)$ & $-0.0102(10)$ & $0.0015(9)$ \\
\hline $\mathrm{C} 22$ & $0.0302(11)$ & $0.0319(11)$ & $0.0626(15)$ & $-0.0058(8)$ & $-0.0118(10)$ & $0.0026(10)$ \\
\hline $\mathrm{C} 23$ & $0.0266(10)$ & $0.0304(10)$ & $0.0658(16)$ & $-0.0067(8)$ & $0.0028(10)$ & $0.0106(10)$ \\
\hline $\mathrm{C} 24$ & $0.0271(9)$ & $0.0267(9)$ & $0.0427(11)$ & $-0.0041(7)$ & $0.0043(8)$ & $0.0059(8)$ \\
\hline $\mathrm{C} 25$ & $0.0235(8)$ & $0.0235(8)$ & $0.0238(8)$ & $-0.0026(7)$ & $0.0071(7)$ & $-0.0018(7)$ \\
\hline $\mathrm{C} 26$ & $0.0268(9)$ & $0.0313(9)$ & $0.0310(10)$ & $-0.0011(7)$ & $0.0062(7)$ & $0.0026(8)$ \\
\hline 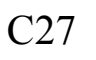 & $0.0368(11)$ & $0.0359(10)$ & $0.0291(10)$ & $-0.0080(8)$ & $0.0109(8)$ & $0.0026(8)$ \\
\hline $\mathrm{C} 28$ & $0.0272(9)$ & $0.0420(11)$ & $0.0367(11)$ & $-0.0117(8)$ & $0.0140(8)$ & $-0.0039(9)$ \\
\hline $\mathrm{C} 29$ & $0.0241(9)$ & $0.0413(11)$ & $0.0403(11)$ & $0.0015(8)$ & $0.0104(8)$ & $-0.0019(9)$ \\
\hline
\end{tabular}




\begin{tabular}{|c|c|c|c|c|c|c|}
\hline $\mathrm{C} 30$ & 0.02 & 78(9) & $3(10)$ & 0.00 & 8) & (8) \\
\hline C31 & $0.0247(8)$ & $0.0202(8)$ & $0.0214(8)$ & $0.0034(6)$ & $0.0017(7)$ & $0.0027(6)$ \\
\hline $\mathrm{C} 32$ & $0.0274(9)$ & $0.0304(9)$ & $0.0322(10)$ & $0.0026(7)$ & $0.0053(8)$ & $-0.0061(8)$ \\
\hline$C 33$ & $.0392(11)$ & $0.0354(10)$ & $.0329(10)$ & $0.0017(9)$ & $0.0056(9)$ & $-0.0104(8)$ \\
\hline $\mathrm{C} 34$ & $0.0381(11)$ & $0.0320(10)$ & $0.0288(10)$ & $-0.0056(8)$ & $-0.0034(8)$ & $-0.0022(8)$ \\
\hline $\mathrm{C} 35$ & $0.0264(9)$ & $0.0341(10)$ & $0.0265(9)$ & $-0.0045(7)$ & $0.0009(7)$ & $0.0048(8)$ \\
\hline $\mathrm{C} 36$ & $0.0248(9)$ & $0.0272(9)$ & $0.0230(8)$ & $0.0003(7)$ & $0.0026(7)$ & $0.0034(7)$ \\
\hline $\mathrm{C}$ & $.0178(7)$ & $0.0200(8)$ & $0.0272(9)$ & $-0.0024(6)$ & $0.0038(6)$ & $-0.0012(6)$ \\
\hline $\mathrm{C} 38$ & $0.0197(8)$ & $0.0168(7)$ & $0.0241(8)$ & $-0.0014(6)$ & $0.0043(6)$ & $-0.0006(6)$ \\
\hline C39 & $0.0214(8)$ & $0.0167(7)$ & $0.0221(8)$ & $-0.0009(6)$ & $-0.0001(6)$ & $-0.0001(6)$ \\
\hline $\mathrm{C} 40$ & $0.0243(8)$ & $0.0285(9)$ & $0.0237(9)$ & $0.0025(7)$ & $0.0015(7)$ & $0.0005(7)$ \\
\hline $\mathrm{C} 41$ & $0.0373(11)$ & $0.0359(10)$ & $0.0227(9)$ & $0.0021(8)$ & $0.0029(8)$ & $0.0035(8)$ \\
\hline $\mathrm{C} 42$ & $0.0383(11)$ & $0.0370(11)$ & $0.0247(9)$ & $0.0006(9)$ & $-0.0075(8)$ & $0.0043(8)$ \\
\hline $\mathrm{C} 43$ & $0.0258(9)$ & $0.0369(11)$ & $0.0340(10)$ & $0.0021(8)$ & $-0.0064(8)$ & $0.0053(8)$ \\
\hline $\mathrm{C} 4$ & $0.0210(8)$ & $0.0296(9)$ & $0.0270(9)$ & $0.0001(7)$ & $0.0001(7)$ & $0.0025(7)$ \\
\hline $\mathrm{C} 45$ & $0.0210(8)$ & $0.0201(8)$ & $0.0314(9)$ & $-0.0008(6)$ & $0.0111(7)$ & $0.0005(7)$ \\
\hline $\mathrm{C} 46$ & $0.0304(9)$ & $0.0251(9)$ & $0.0317(10)$ & $0.0024(7)$ & $0.0103(8)$ & $-0.0019(7)$ \\
\hline $\mathrm{C} 47$ & $0.0421(11)$ & $0.0275(9)$ & $0.0376(11)$ & $0.0051(8)$ & $0.0193(9)$ & $-0.0026(8)$ \\
\hline $\mathrm{C} 48$ & $0.0368(11)$ & $0.0282(10)$ & $0.0523(13)$ & $0.0089(8)$ & $0.0246(10)$ & $0.0029(9)$ \\
\hline C49 & $0.0245(9)$ & $0.0370(11)$ & $0.0532(13)$ & $0.0070(8)$ & $0.0086(9)$ & $0.0066(10)$ \\
\hline $\mathrm{C} 50$ & $0.0250(9)$ & $0.0287(9)$ & $0.0381(11)$ & $0.0019(7)$ & $0.0047(8)$ & $8(8)$ \\
\hline $\mathrm{C} 5$ & $0.0246(8)$ & $0.0192(8)$ & $0.0234(8)$ & $-0.0013(6)$ & $0.0040(7)$ & $-0.0030(6)$ \\
\hline C52 & $0.0255(9)$ & $0.0480(12)$ & $0.0285(10)$ & $0.0057(8)$ & $0.0052(8)$ & $0.0042(9)$ \\
\hline C53 & $0.0343(11)$ & $0.0671(16)$ & $0.0349(11)$ & $0.0171(11)$ & $0.0017(9)$ & $0.0069(11)$ \\
\hline $\mathrm{C} 5$ & $0.0516(13)$ & 1(12) & $3(10)$ & $0.0083(10)$ & $0.0019(9)$ & $5(9)$ \\
\hline C55 & $0.0455(12)$ & $0.0295(10)$ & $0.0279(10)$ & $-0.0043(8)$ & $0.0110(9)$ & $0.0021(8)$ \\
\hline $\mathrm{C} 56$ & $0.0296(9)$ & $0.0271(9)$ & $0.0295(9)$ & $-0.0030(7)$ & $0.0080(7)$ & $-0.0005(7)$ \\
\hline C57 & $0.0233(8)$ & $0.0184(8)$ & $0.0241(8)$ & $-0.0047(6)$ & $0.0031(7)$ & $-0.0009(6)$ \\
\hline $\mathrm{C} 5$ & $0.0253(9)$ & $0.0315(10)$ & $0.0306(10)$ & $-0.0006(7)$ & $0.0052(7)$ & $0.0064(8)$ \\
\hline C59 & $0.0307(10)$ & $0.0347(10)$ & $0.0345(10)$ & $0.0036(8)$ & $-0.0012(8)$ & $0.0076(8)$ \\
\hline C60 & $0.0447(12)$ & $0.0300(10)$ & $0.0261(9)$ & $-0.0051(8)$ & $0.0005(8)$ & $0.0071(8)$ \\
\hline C6 & $0.0506(12)$ & $0.0284(10)$ & $0.0310(10)$ & $-0.0021(9)$ & $0.0187(9)$ & $0.0017(8)$ \\
\hline C62 & $0.0356(10)$ & $0.0253(9)$ & $0.0332(10)$ & $0.0019(8)$ & $0.0138(8)$ & $0.0020(8)$ \\
\hline C63 & $0.0236(8)$ & $0.0187(8)$ & $0.0237(8)$ & $-0.0050(6)$ & $0.0024(6)$ & $0.0008(6)$ \\
\hline C64 & $0.0272(9)$ & $0.0212(8)$ & $0.0317(10)$ & $-0.0030(7)$ & $0.0042(7)$ & $-0.0019(7)$ \\
\hline C65 & $0.0425(11)$ & $0.0271(9)$ & $0.0316(10)$ & $-0.0047(8)$ & $0.0063(9)$ & $-0.0070(8)$ \\
\hline C66 & $0.0448(12)$ & $0.0360(11)$ & $0.0286(10)$ & $-0.0110(9)$ & $-0.0034(9)$ & $-0.0049(8)$ \\
\hline C67 & $0.0293(10)$ & $0.0397(11)$ & $0.0351(11)$ & $-0.0053(8)$ & $-0.0050(8)$ & $-0.0003(9)$ \\
\hline C68 & $0.0242(9)$ & $0.0274(9)$ & $0.0314(10)$ & $-0.0029(7)$ & $0.0025(7)$ & $-0.0001(7)$ \\
\hline
\end{tabular}




$\begin{array}{lllllll}\text { C69 } & 0.0174(7) & 0.0229(8) & 0.0209(8) & -0.0008(6) & 0.0051(6) & -0.0026(6) \\ \text { C70 } & 0.0250(9) & 0.0231(8) & 0.0306(9) & 0.0023(7) & -0.0011(7) & -0.0035(7) \\ \text { C71 } & 0.0294(10) & 0.0287(9) & 0.0370(11) & 0.0060(8) & -0.0020(8) & 0.0004(8) \\ \text { C72 } & 0.0267(9) & 0.0421(11) & 0.0282(10) & 0.0012(8) & -0.0044(8) & -0.0022(8) \\ \text { C73 } & 0.0347(10) & 0.0358(10) & 0.0251(9) & -0.0107(8) & -0.0002(8) & -0.0066(8) \\ \text { C74 } & 0.0309(9) & 0.0245(8) & 0.0225(8) & -0.0067(7) & 0.0033(7) & -0.0025(7) \\ \text { C75 } & 0.0276(9) & 0.0196(8) & 0.0254(9) & -0.0022(7) & 0.0086(7) & -0.0027(7) \\ \text { C76 } & 0.0362(10) & 0.0239(9) & 0.0359(10) & 0.0029(8) & 0.0102(8) & -0.0037(8) \\ \text { C77 } & 0.0537(13) & 0.0230(9) & 0.0456(12) & -0.0029(9) & 0.0204(11) & -0.0093(9) \\ \text { C78 } & 0.0534(14) & 0.0336(11) & 0.0380(12) & -0.0134(10) & 0.0139(10) & -0.0146(9) \\ \text { C79 } & 0.0377(11) & 0.0408(11) & 0.0303(10) & -0.0084(9) & 0.0040(8) & -0.0083(9) \\ \text { C80 } & 0.0328(10) & 0.0268(9) & 0.0246(9) & -0.0010(7) & 0.0075(7) & -0.0026(7) \\ \text { C81 } & 0.0197(8) & 0.0199(8) & 0.0277(9) & 0.0030(6) & 0.0054(7) & 0.0032(7) \\ \text { C82 } & 0.0334(10) & 0.0226(8) & 0.0288(9) & 0.0011(7) & 0.0051(8) & 0.0029(7) \\ \text { C83 } & 0.0412(11) & 0.0342(10) & 0.0292(10) & 0.0021(9) & 0.0036(8) & 0.0039(8) \\ \text { C84 } & 0.0458(12) & 0.0355(11) & 0.0344(11) & 0.0057(9) & 0.0093(9) & 0.0150(9) \\ \text { C85 } & 0.0447(12) & 0.0233(9) & 0.0477(12) & 0.0062(8) & 0.0154(10) & 0.0123(9) \\ \text { C86 } & 0.0331(10) & 0.0206(8) & 0.0376(10) & 0.0021(7) & 0.0115(8) & 0.0029(7) \\ \text { C87 } & 0.0212(8) & 0.0143(7) & 0.0330(9) & 0.0044(6) & 0.0082(7) & 0.0007(6) \\ \text { C88 } & 0.0232(8) & 0.0220(8) & 0.0367(10) & 0.0043(7) & 0.0054(7) & 0.0022(7) \\ \text { C89 } & 0.0201(9) & 0.0295(10) & 0.0529(13) & 0.0038(7) & 0.0046(8) & 0.0047(9) \\ \text { C90 } & 0.0275(10) & 0.0280(10) & 0.0653(15) & 0.0061(8) & 0.0223(10) & 0.0097(10) \\ \text { C91 } & 0.0425(12) & 0.0284(10) & 0.0461(12) & 0.0040(8) & 0.0262(10) & 0.0039(9) \\ \text { C92 } & 0.0334(10) & 0.0225(8) & 0.0336(10) & 0.0014(7) & 0.0124(8) & 0.0003(7)\end{array}$

The general temperature factor expression: $\exp \left(-2 \pi^{2}\left(\mathrm{a}^{*} \mathrm{U}_{11} \mathrm{~h}^{2}+\mathrm{b} * 2 \mathrm{U}_{22} \mathrm{k}^{2}+\mathrm{c}^{* 2} \mathrm{U}_{33} \mathrm{1}^{2}+\right.\right.$ $\left.\left.2 a * b * U_{12} h k+2 a * c * U_{13} h l+2 b * c * U_{23} k l\right)\right)$

Table S4-4. Bond lengths $(\AA)$

$\begin{array}{llllll}\text { atom } & \text { atom } & \text { distance } & \text { atom } & \text { atom } & \text { distance } \\ \mathrm{Pd} 1 & \mathrm{Pd} 2 & 2.9106(8) & \mathrm{Pd} 1 & \mathrm{P} 1 & 2.2307(6) \\ \mathrm{Pd} 1 & \mathrm{Si} 1 & 2.3331(7) & \mathrm{Pd} 1 & \mathrm{C} 1 & 1.9790(17) \\ \mathrm{Pd} 2 & \mathrm{Pd} 3 & 2.8972(6) & \mathrm{Pd} 2 & \mathrm{P} 1 & 2.2871(7) \\ \mathrm{Pd} 2 & \mathrm{P} 2 & 2.2811(6) & \mathrm{Pd} 2 & \mathrm{P} 3 & 2.2874(7) \\ \mathrm{Pd} 3 & \mathrm{P} 2 & 2.2184(7) & \mathrm{Pd} 3 & \mathrm{Si} & 2.3326(6) \\ \mathrm{Pd} 3 & \mathrm{C} 6 & 1.9815(17) & \mathrm{P} 1 & \mathrm{C} 12 & 1.8224(18) \\ \mathrm{P} 1 & \mathrm{C} 39 & 1.8138(17) & \mathrm{P} 2 & \mathrm{C} 38 & 1.8290(17) \\ \mathrm{P} 2 & \mathrm{C} 69 & 1.8173(16) & \mathrm{P} 3 & \mathrm{C} 75 & 1.8265(17) \\ \mathrm{P} 3 & \mathrm{C} 81 & 1.8279(18) & \mathrm{P} 3 & \mathrm{C} 87 & 1.8318(18) \\ & & & \mathrm{S}-36 & & \end{array}$




\begin{tabular}{|c|c|c|c|c|c|}
\hline Sil & $\mathrm{C} 11$ & $1.8866(19)$ & Si1 & $\mathrm{C} 13$ & $1.8956(17)$ \\
\hline Sil & $\mathrm{C} 19$ & $1.8945(17)$ & $\mathrm{Si} 2$ & $\mathrm{C} 11$ & $1.8621(18)$ \\
\hline $\mathrm{Si} 2$ & $\mathrm{C} 12$ & $1.8818(17)$ & $\mathrm{Si} 2$ & $\mathrm{C} 25$ & $1.881(2)$ \\
\hline $\mathrm{Si} 2$ & $\mathrm{C} 31$ & $1.8817(17)$ & $\mathrm{Si} 3$ & $\mathrm{C} 37$ & $1.9053(17)$ \\
\hline $\mathrm{Si} 3$ & $\mathrm{C} 45$ & $1.8946(18)$ & $\mathrm{Si} 3$ & $\mathrm{C} 51$ & $1.8887(17)$ \\
\hline $\mathrm{Si} 4$ & C37 & $1.8599(18)$ & $\mathrm{Si} 4$ & $\mathrm{C} 38$ & $1.8805(18)$ \\
\hline $\mathrm{Si} 4$ & $\mathrm{C} 57$ & $1.8717(17)$ & $\mathrm{Si} 4$ & C63 & $1.8789(17)$ \\
\hline N1 & $\mathrm{C} 1$ & $1.146(2)$ & N1 & $\mathrm{C} 2$ & $1.457(3)$ \\
\hline N1 & C93 & $1.546(12)$ & $\mathrm{N} 2$ & $\mathrm{C} 6$ & $1.154(2)$ \\
\hline N2 & $\mathrm{C} 7$ & $1.453(2)$ & $\mathrm{C} 2$ & $\mathrm{C} 3$ & $1.516(4)$ \\
\hline $\mathrm{C} 2$ & $\mathrm{C} 4$ & $1.534(4)$ & $\mathrm{C} 2$ & $\mathrm{C} 5$ & $1.525(5)$ \\
\hline $\mathrm{C} 2$ & C93 & $0.720(13)$ & $\mathrm{C} 2$ & C95 & $1.27(2)$ \\
\hline $\mathrm{C} 3$ & C93 & $1.450(11)$ & $\mathrm{C} 4$ & C95 & $1.82(3)$ \\
\hline $\mathrm{C} 5$ & C93 & $1.328(12)$ & $\mathrm{C} 5$ & C94 & $1.786(14)$ \\
\hline $\mathrm{C} 5$ & C95 & $0.76(2)$ & $\mathrm{C} 7$ & $\mathrm{C} 8$ & $1.517(3)$ \\
\hline $\mathrm{C} 7$ & $\mathrm{C} 9$ & $1.522(3)$ & $\mathrm{C} 7$ & $\mathrm{C} 10$ & $1.514(3)$ \\
\hline $\mathrm{C} 13$ & $\mathrm{C} 14$ & $1.396(3)$ & $\mathrm{C} 13$ & $\mathrm{C} 18$ & $1.396(3)$ \\
\hline $\mathrm{C} 14$ & $\mathrm{C} 15$ & $1.389(3)$ & $\mathrm{C} 15$ & $\mathrm{C} 16$ & $1.377(3)$ \\
\hline $\mathrm{C} 16$ & $\mathrm{C} 17$ & $1.377(3)$ & $\mathrm{C} 17$ & $\mathrm{C} 18$ & $1.395(3)$ \\
\hline C19 & $\mathrm{C} 20$ & $1.382(3)$ & $\mathrm{C} 19$ & $\mathrm{C} 24$ & $1.403(3)$ \\
\hline $\mathrm{C} 20$ & $\mathrm{C} 21$ & $1.393(3)$ & $\mathrm{C} 21$ & $\mathrm{C} 22$ & $1.378(4)$ \\
\hline $\mathrm{C} 22$ & $\mathrm{C} 23$ & $1.360(4)$ & $\mathrm{C} 23$ & $\mathrm{C} 24$ & $1.387(3)$ \\
\hline $\mathrm{C} 25$ & $\mathrm{C} 26$ & $1.401(3)$ & $\mathrm{C} 25$ & $\mathrm{C} 30$ & $1.391(3)$ \\
\hline $\mathrm{C} 26$ & $\mathrm{C} 27$ & $1.388(3)$ & $\mathrm{C} 27$ & $\mathrm{C} 28$ & $1.378(3)$ \\
\hline $\mathrm{C} 28$ & $\mathrm{C} 29$ & $1.371(3)$ & $\mathrm{C} 29$ & $\mathrm{C} 30$ & $1.385(3)$ \\
\hline C31 & $\mathrm{C} 32$ & $1.388(3)$ & $\mathrm{C} 31$ & $\mathrm{C} 36$ & $1.402(3)$ \\
\hline $\mathrm{C} 32$ & C33 & $1.393(3)$ & $\mathrm{C} 33$ & C34 & $1.376(3)$ \\
\hline C34 & C35 & $1.386(3)$ & $\mathrm{C} 35$ & C36 & $1.385(3)$ \\
\hline C39 & $\mathrm{C} 40$ & $1.397(3)$ & C39 & $\mathrm{C} 44$ & $1.393(2)$ \\
\hline $\mathrm{C} 40$ & $\mathrm{C} 41$ & $1.384(3)$ & $\mathrm{C} 41$ & $\mathrm{C} 42$ & $1.381(3)$ \\
\hline $\mathrm{C} 42$ & $\mathrm{C} 43$ & $1.383(3)$ & $\mathrm{C} 43$ & $\mathrm{C} 44$ & $1.391(3)$ \\
\hline $\mathrm{C} 45$ & $\mathrm{C} 46$ & $1.393(3)$ & $\mathrm{C} 45$ & C50 & $1.398(3)$ \\
\hline $\mathrm{C} 46$ & $\mathrm{C} 47$ & $1.391(3)$ & $\mathrm{C} 47$ & $\mathrm{C} 48$ & $1.374(3)$ \\
\hline C48 & $\mathrm{C} 49$ & $1.384(3)$ & $\mathrm{C} 49$ & $\mathrm{C} 50$ & $1.385(3)$ \\
\hline C51 & C52 & $1.391(3)$ & $\mathrm{C} 51$ & C56 & $1.398(3)$ \\
\hline C52 & C53 & $1.391(3)$ & C53 & C54 & $1.380(4)$ \\
\hline C54 & $\mathrm{C} 55$ & $1.376(3)$ & $\mathrm{C} 55$ & $\mathrm{C} 56$ & $1.384(3)$ \\
\hline $\mathrm{C} 57$ & C58 & $1.395(2)$ & $\mathrm{C} 57$ & C62 & $1.390(3)$ \\
\hline C58 & $\mathrm{C} 59$ & $1.388(3)$ & $\mathrm{C} 59$ & C60 & $1.380(3)$ \\
\hline
\end{tabular}




$\begin{array}{llllll}\text { C60 } & \text { C61 } & 1.378(3) & \text { C61 } & \text { C62 } & 1.388(3) \\ \text { C63 } & \text { C64 } & 1.402(3) & \text { C63 } & \text { C68 } & 1.389(2) \\ \text { C64 } & \text { C65 } & 1.386(3) & \mathrm{C} 65 & \mathrm{C} 66 & 1.383(3) \\ \text { C66 } & \text { C67 } & 1.374(3) & \mathrm{C} 67 & \mathrm{C} 68 & 1.388(3) \\ \text { C69 } & \text { C70 } & 1.399(2) & \mathrm{C} 69 & \mathrm{C} 74 & 1.390(2) \\ \mathrm{C} 70 & \mathrm{C} 71 & 1.380(3) & \mathrm{C} 71 & \mathrm{C} 72 & 1.385(3) \\ \mathrm{C} 72 & \mathrm{C} 73 & 1.377(3) & \mathrm{C} 73 & \mathrm{C} 74 & 1.395(2) \\ \mathrm{C} 75 & \mathrm{C} 76 & 1.397(3) & \mathrm{C} 75 & \mathrm{C} 80 & 1.383(2) \\ \mathrm{C} 76 & \mathrm{C} 77 & 1.383(3) & \mathrm{C} 77 & \mathrm{C} 78 & 1.379(3) \\ \mathrm{C} 78 & \mathrm{C} 79 & 1.379(3) & \mathrm{C} 79 & \mathrm{C} 80 & 1.392(3) \\ \mathrm{C} 81 & \mathrm{C} 82 & 1.391(3) & \mathrm{C} 81 & \mathrm{C} 86 & 1.394(2) \\ \mathrm{C} 82 & \mathrm{C} 83 & 1.381(3) & \mathrm{C} 83 & \mathrm{C} 84 & 1.382(3) \\ \mathrm{C} 84 & \mathrm{C} 85 & 1.370(3) & \mathrm{C} 85 & \mathrm{C} 86 & 1.388(3) \\ \mathrm{C} 87 & \mathrm{C} 88 & 1.388(2) & \mathrm{C} 87 & \mathrm{C} 92 & 1.389(3) \\ \mathrm{C} 88 & \mathrm{C} 89 & 1.395(3) & \mathrm{C} 89 & \mathrm{C} 90 & 1.383(4) \\ \mathrm{C} 90 & \mathrm{C} 91 & 1.383(3) & \mathrm{C} 91 & \mathrm{C} 92 & 1.387(3) \\ \mathrm{C} 93 & \mathrm{C} 94 & 1.495(19) & \mathrm{C} 93 & \mathrm{C} 95 & 1.46(3)\end{array}$

Table S4-5. Bond angles $\left({ }^{\circ}\right)$

$\begin{array}{llllllll}\text { atom } & \text { atom } & \text { atom } & \text { angle } & \text { atom } & \text { atom } & \text { atom } & \text { angle } \\ \mathrm{Pd} 2 & \mathrm{Pd} 1 & \mathrm{P} 1 & 50.745(18) & \mathrm{Pd} 2 & \mathrm{Pd} 1 & \mathrm{Si} 1 & 129.60(2) \\ \mathrm{Pd} 2 & \mathrm{Pd} 1 & \mathrm{C} 1 & 121.91(5) & \mathrm{P} 1 & \mathrm{Pd} 1 & \mathrm{Si} & 88.55(2) \\ \mathrm{P} 1 & \mathrm{Pd} 1 & \mathrm{C} 1 & 171.36(5) & \mathrm{Si} 1 & \mathrm{Pd} 1 & \mathrm{C} 1 & 94.95(5) \\ \mathrm{Pd} 1 & \mathrm{Pd} 2 & \mathrm{Pd} 3 & 60.686(16) & \mathrm{Pd} 1 & \mathrm{Pd} 2 & \mathrm{P} 1 & 49.046(17) \\ \mathrm{Pd} 1 & \mathrm{Pd} 2 & \mathrm{P} 2 & 101.21(2) & \mathrm{Pd} 1 & \mathrm{Pd} 2 & \mathrm{P} 3 & 155.329(19) \\ \mathrm{Pd} 3 & \mathrm{Pd} 2 & \mathrm{P} 1 & 98.60(2) & \mathrm{Pd} 3 & \mathrm{Pd} 2 & \mathrm{P} 2 & 48.975(17) \\ \mathrm{Pd} 3 & \mathrm{Pd} 2 & \mathrm{P} 3 & 139.19(3) & \mathrm{P} 1 & \mathrm{Pd} 2 & \mathrm{P} 2 & 147.13(3) \\ \mathrm{P} 1 & \mathrm{Pd} 2 & \mathrm{P} 3 & 106.80(2) & \mathrm{P} 2 & \mathrm{Pd} 2 & \mathrm{P} 3 & 103.43(3) \\ \mathrm{Pd} 2 & \mathrm{Pd} 3 & \mathrm{P} 2 & 50.874(17) & \mathrm{Pd} 2 & \mathrm{Pd} 3 & \mathrm{Si} 3 & 134.39(3) \\ \mathrm{Pd} 2 & \mathrm{Pd} 3 & \mathrm{C} 6 & 122.59(5) & \mathrm{P} 2 & \mathrm{Pd} 3 & \mathrm{Si} 3 & 89.81(2) \\ \mathrm{P} 2 & \mathrm{Pd} 3 & \mathrm{C} 6 & 173.24(5) & \mathrm{Si} & \mathrm{Pd} 3 & \mathrm{C} 6 & 95.30(6) \\ \mathrm{Pd} 1 & \mathrm{P} 1 & \mathrm{Pd} 2 & 80.21(2) & \mathrm{Pd} 1 & \mathrm{P} 1 & \mathrm{C} 12 & 121.68(6) \\ \mathrm{Pd} 1 & \mathrm{P} 1 & \mathrm{C} 39 & 115.99(6) & \mathrm{Pd} 2 & \mathrm{P} 1 & \mathrm{C} 12 & 119.23(6) \\ \mathrm{Pd} 2 & \mathrm{P} 1 & \mathrm{C} 39 & 113.33(6) & \mathrm{C} 12 & \mathrm{P} 1 & \mathrm{C} 39 & 105.34(8) \\ \mathrm{Pd} 2 & \mathrm{P} 2 & \mathrm{Pd} 3 & 80.15(2) & \mathrm{Pd} 2 & \mathrm{P} 2 & \mathrm{C} 38 & 115.40(6) \\ \mathrm{Pd} 2 & \mathrm{P} 2 & \mathrm{C} 69 & 119.41(6) & \mathrm{Pd} 3 & \mathrm{P} 2 & \mathrm{C} 38 & 120.11(6) \\ \mathrm{Pd} 3 & \mathrm{P} 2 & \mathrm{C} 69 & 113.23(6) & \mathrm{C} 38 & \mathrm{P} 2 & \mathrm{C} 69 & 107.19(8) \\ \mathrm{Pd} 2 & \mathrm{P} 3 & \mathrm{C} 75 & 119.98(6) & \mathrm{Pd} 2 & \mathrm{P} 3 & \mathrm{C} 81 & 107.16(6) \\ \mathrm{Pd} 2 & \mathrm{P} 3 & \mathrm{C} 87 & 117.76(6) & \mathrm{C} 75 & \mathrm{P} 3 & \mathrm{C} 81 & 105.58(8) \\ & & & & \mathrm{S}-38 & & & \end{array}$




\begin{tabular}{|c|c|c|c|c|c|c|c|}
\hline $\mathrm{C} 75$ & P3 & $\mathrm{C} 87$ & $101.57(9)$ & $\mathrm{C} 81$ & P3 & $\mathrm{C} 87$ & $103.01(8)$ \\
\hline $\mathrm{Pd} 1$ & Si1 & $\mathrm{C} 11$ & $105.50(6)$ & Pd1 & Sil & $\mathrm{C} 13$ & $115.44(6)$ \\
\hline Pd1 & Sil & C19 & $119.66(6)$ & $\mathrm{C} 11$ & Sil & $\mathrm{C} 13$ & $105.08(8)$ \\
\hline $\mathrm{C} 11$ & Si1 & C19 & $105.35(8)$ & $\mathrm{C} 13$ & Sil & $\mathrm{C} 19$ & $104.51(7)$ \\
\hline $\mathrm{C} 11$ & $\mathrm{Si} 2$ & $\mathrm{C} 12$ & $111.98(8)$ & $\mathrm{C} 11$ & $\mathrm{Si} 2$ & $\mathrm{C} 25$ & $110.51(8)$ \\
\hline C11 & $\mathrm{Si} 2$ & $\mathrm{C} 31$ & $108.48(8)$ & $\mathrm{C} 12$ & $\mathrm{Si} 2$ & $\mathrm{C} 25$ & $105.63(7)$ \\
\hline $\mathrm{C} 12$ & $\mathrm{Si} 2$ & $\mathrm{C} 31$ & $111.04(7)$ & $\mathrm{C} 25$ & $\mathrm{Si} 2$ & $\mathrm{C} 31$ & $109.17(8)$ \\
\hline $\mathrm{Pd} 3$ & $\mathrm{Si} 3$ & $\mathrm{C} 37$ & $120.75(6)$ & $\mathrm{Pd} 3$ & $\mathrm{Si} 3$ & $\mathrm{C} 45$ & $110.21(6)$ \\
\hline $\mathrm{Pd} 3$ & $\mathrm{Si} 3$ & $\mathrm{C} 51$ & $108.86(6)$ & $\mathrm{C} 37$ & $\mathrm{Si} 3$ & $\mathrm{C} 45$ & $104.86(7)$ \\
\hline $\mathrm{C} 37$ & $\mathrm{Si} 3$ & C51 & $104.63(7)$ & $\mathrm{C} 45$ & $\mathrm{Si} 3$ & $\mathrm{C} 51$ & $106.63(8)$ \\
\hline C37 & $\mathrm{Si} 4$ & $\mathrm{C} 38$ & $108.99(8)$ & $\mathrm{C} 37$ & $\mathrm{Si} 4$ & $\mathrm{C} 57$ & $112.95(8)$ \\
\hline $\mathrm{C} 37$ & $\mathrm{Si} 4$ & C63 & $112.60(7)$ & C38 & $\mathrm{Si} 4$ & $\mathrm{C} 57$ & $108.67(8)$ \\
\hline $\mathrm{C} 38$ & $\mathrm{Si} 4$ & C63 & $107.33(8)$ & C57 & $\mathrm{Si} 4$ & C63 & $106.07(8)$ \\
\hline $\mathrm{C} 1$ & N1 & $\mathrm{C} 2$ & $173.8(2)$ & $\mathrm{C} 1$ & N1 & C93 & $158.3(5)$ \\
\hline $\mathrm{C} 2$ & N1 & C93 & $27.5(5)$ & C6 & $\mathrm{N} 2$ & $\mathrm{C} 7$ & $169.46(19)$ \\
\hline Pd1 & $\mathrm{C} 1$ & $\mathrm{~N} 1$ & $177.00(16)$ & N1 & $\mathrm{C} 2$ & $\mathrm{C} 3$ & $107.76(19)$ \\
\hline N1 & $\mathrm{C} 2$ & $\mathrm{C} 4$ & $107.9(2)$ & N1 & $\mathrm{C} 2$ & $\mathrm{C} 5$ & $106.3(2)$ \\
\hline N1 & $\mathrm{C} 2$ & C93 & $83.1(9)$ & N1 & $\mathrm{C} 2$ & C95 & $118.6(11)$ \\
\hline $\mathrm{C} 3$ & $\mathrm{C} 2$ & $\mathrm{C} 4$ & $109.4(2)$ & $\mathrm{C} 3$ & $\mathrm{C} 2$ & $\mathrm{C} 5$ & $115.0(3)$ \\
\hline $\mathrm{C} 3$ & $\mathrm{C} 2$ & C93 & $70.9(9)$ & $\mathrm{C} 3$ & $\mathrm{C} 2$ & $\mathrm{C} 95$ & $126.9(12)$ \\
\hline $\mathrm{C} 4$ & $\mathrm{C} 2$ & $\mathrm{C} 5$ & $110.1(3)$ & $\mathrm{C} 4$ & $\mathrm{C} 2$ & C93 & 167.7(9) \\
\hline $\mathrm{C} 4$ & $\mathrm{C} 2$ & $\mathrm{C} 95$ & $80.5(11)$ & $\mathrm{C} 5$ & $\mathrm{C} 2$ & C93 & $60.5(9)$ \\
\hline $\mathrm{C} 5$ & $\mathrm{C} 2$ & C95 & $29.6(11)$ & C93 & $\mathrm{C} 2$ & C95 & $89.6(14)$ \\
\hline $\mathrm{C} 2$ & $\mathrm{C} 3$ & C93 & $28.0(5)$ & $\mathrm{C} 2$ & $\mathrm{C} 4$ & $\mathrm{C} 95$ & $43.5(7)$ \\
\hline $\mathrm{C} 2$ & $\mathrm{C} 5$ & C93 & $28.2(6)$ & $\mathrm{C} 2$ & $\mathrm{C} 5$ & C94 & $83.2(5)$ \\
\hline $\mathrm{C} 2$ & $\mathrm{C} 5$ & $\mathrm{C} 95$ & $56.3(18)$ & C93 & $\mathrm{C} 5$ & C94 & $55.0(7)$ \\
\hline C93 & $\mathrm{C} 5$ & C95 & $84.0(19)$ & C94 & $\mathrm{C} 5$ & C95 & $137.6(19)$ \\
\hline $\mathrm{Pd} 3$ & C6 & N2 & $179.43(15)$ & $\mathrm{N} 2$ & $\mathrm{C} 7$ & $\mathrm{C} 8$ & $107.07(16)$ \\
\hline N2 & $\mathrm{C} 7$ & C9 & $106.94(16)$ & $\mathrm{N} 2$ & $\mathrm{C} 7$ & $\mathrm{C} 10$ & $108.62(17)$ \\
\hline $\mathrm{C} 8$ & $\mathrm{C} 7$ & C9 & 109.91(19) & $\mathrm{C} 8$ & $\mathrm{C} 7$ & $\mathrm{C} 10$ & $112.43(19)$ \\
\hline C9 & $\mathrm{C} 7$ & $\mathrm{C} 10$ & $111.60(17)$ & Sil & $\mathrm{C} 11$ & $\mathrm{Si} 2$ & $114.85(9)$ \\
\hline $\mathrm{P} 1$ & $\mathrm{C} 12$ & $\mathrm{Si} 2$ & $114.34(8)$ & Sil & $\mathrm{C} 13$ & $\mathrm{C} 14$ & $121.82(13)$ \\
\hline Sil & $\mathrm{C} 13$ & $\mathrm{C} 18$ & $121.37(14)$ & $\mathrm{C} 14$ & $\mathrm{C} 13$ & $\mathrm{C} 18$ & $116.79(16)$ \\
\hline $\mathrm{C} 13$ & $\mathrm{C} 14$ & $\mathrm{C} 15$ & $121.72(18)$ & $\mathrm{C} 14$ & $\mathrm{C} 15$ & $\mathrm{C} 16$ & $120.1(2)$ \\
\hline $\mathrm{C} 15$ & $\mathrm{C} 16$ & $\mathrm{C} 17$ & $119.83(19)$ & $\mathrm{C} 16$ & $\mathrm{C} 17$ & $\mathrm{C} 18$ & $119.8(2)$ \\
\hline $\mathrm{C} 13$ & $\mathrm{C} 18$ & $\mathrm{C} 17$ & 121.71(19) & Sil & $\mathrm{C} 19$ & $\mathrm{C} 20$ & $122.13(15)$ \\
\hline Sil & C19 & $\mathrm{C} 24$ & $121.25(14)$ & $\mathrm{C} 20$ & C19 & $\mathrm{C} 24$ & $116.60(16)$ \\
\hline C19 & $\mathrm{C} 20$ & $\mathrm{C} 21$ & $121.3(2)$ & $\mathrm{C} 20$ & $\mathrm{C} 21$ & $\mathrm{C} 22$ & $120.3(2)$ \\
\hline $\mathrm{C} 21$ & $\mathrm{C} 22$ & $\mathrm{C} 23$ & $119.8(2)$ & $\mathrm{C} 22$ & $\mathrm{C} 23$ & $\mathrm{C} 24$ & $119.9(2)$ \\
\hline
\end{tabular}




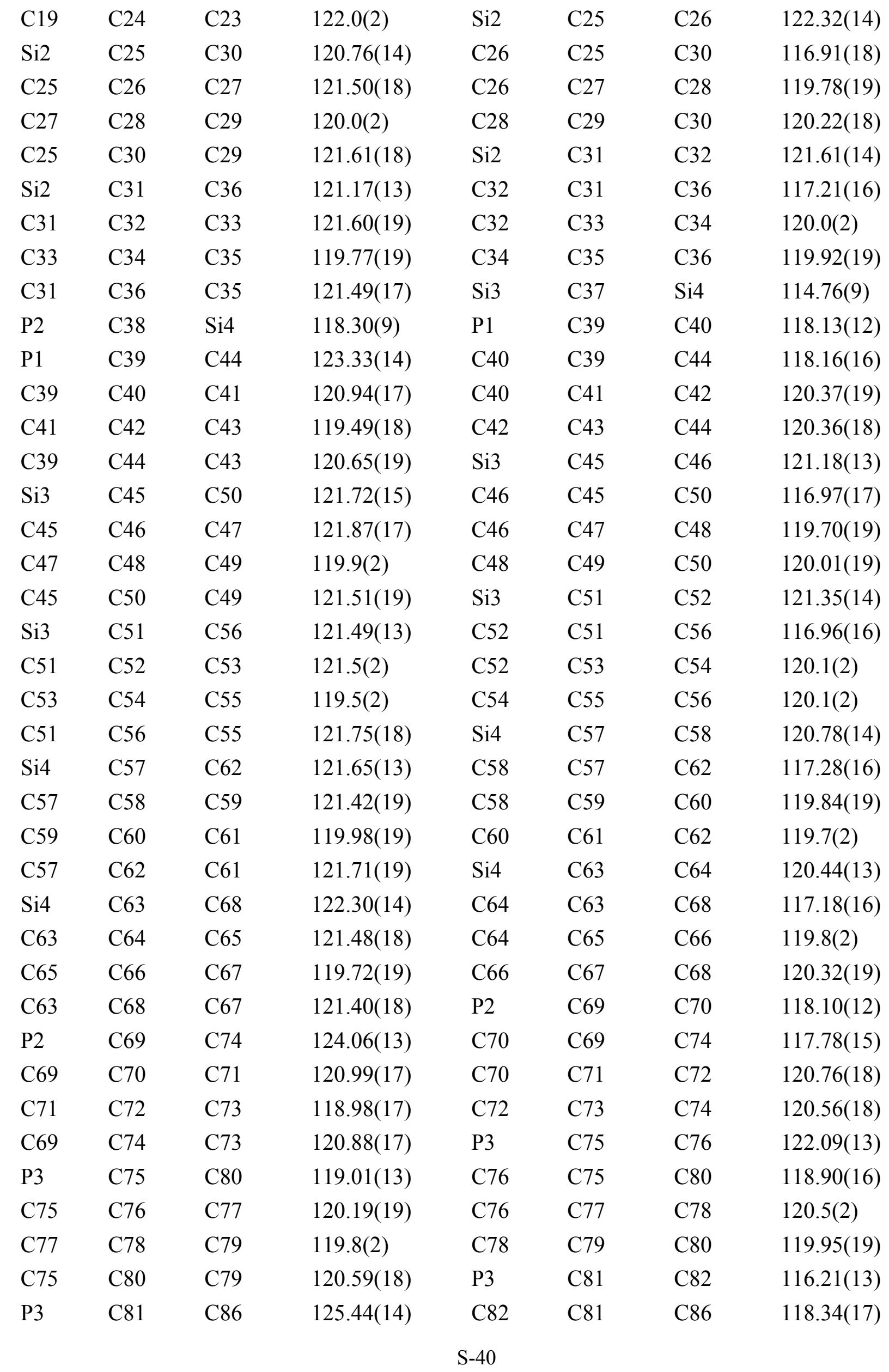




$\begin{array}{llllllll}\text { C81 } & \text { C82 } & \text { C83 } & 121.35(18) & \text { C82 } & \text { C83 } & \text { C84 } & 119.63(19) \\ \text { C83 } & \text { C84 } & \text { C85 } & 119.8(2) & \text { C84 } & \text { C85 } & \text { C86 } & 121.05(18) \\ \text { C81 } & \text { C86 } & \text { C85 } & 119.82(18) & \text { P3 } & \text { C87 } & \text { C88 } & 121.71(16) \\ \text { P3 } & \text { C87 } & \text { C92 } & 119.22(13) & \text { C88 } & \text { C87 } & \text { C92 } & 118.69(17) \\ \text { C87 } & \text { C88 } & \text { C89 } & 120.5(2) & \text { C88 } & \text { C89 } & \text { C90 } & 120.13(19) \\ \text { C89 } & \text { C90 } & \text { C91 } & 119.6(2) & \text { C90 } & \text { C91 } & \text { C92 } & 120.2(2) \\ \text { C87 } & \text { C92 } & \text { C91 } & 120.89(17) & \text { N1 } & \text { C93 } & \text { C2 } & 69.3(9) \\ \text { N1 } & \text { C93 } & \text { C3 } & 106.5(7) & \text { N1 } & \text { C93 } & \text { C5 } & 112.1(8) \\ \text { N1 } & \text { C93 } & \text { C94 } & 116.1(9) & \text { N1 } & \text { C93 } & \text { C95 } & 102.9(12) \\ \text { C2 } & \text { C93 } & \text { C3 } & 81.1(10) & \text { C2 } & \text { C93 } & \text { C5 } & 91.4(11) \\ \text { C2 } & \text { C93 } & \text { C94 } & 169.5(14) & \text { C2 } & \text { C93 } & \text { C95 } & 60.8(13) \\ \text { C3 } & \text { C93 } & \text { C5 } & 134.9(8) & \text { C3 } & \text { C93 } & \text { C94 } & 104.9(9) \\ \text { C3 } & \text { C93 } & \text { C95 } & 118.3(12) & \text { C5 } & \text { C93 } & \text { C94 } & 78.3(8) \\ \text { C5 } & \text { C93 } & \text { C95 } & 31.1(11) & \text { C94 } & \text { C93 } & \text { C95 } & 108.7(13) \\ \text { C5 } & \text { C94 } & \text { C93 } & 46.7(6) & \text { C2 } & \text { C95 } & \text { C4 } & 56.1(10) \\ \text { C2 } & \text { C95 } & \text { C5 } & 94(2) & \text { C2 } & \text { C95 } & \text { C93 } & 29.6(7) \\ \text { C4 } & \text { C95 } & \text { C5 } & 150(2) & \text { C4 } & \text { C95 } & \text { C93 } & 85.5(13) \\ \text { C5 } & \text { C95 } & \text { C93 } & 64.9(17) & & & & \end{array}$

Table S4-6. Torsion Angles $\left({ }^{\circ}\right)$

(Those having bond angles $>160$ or $<20$ degrees are excluded.)

atom1 atom2 atom3 atom4 angle

$\begin{array}{lllll}\mathrm{Pd} 2 & \mathrm{Pd} 1 & \mathrm{P} 1 & \mathrm{Pd} 2 & 0.000(11)\end{array}$

$\begin{array}{lllll}\mathrm{Pd} 2 & \mathrm{Pd} 1 & \mathrm{P} 1 & \mathrm{C} 39 & -111.28(3)\end{array}$

P1 Pd1 Pd2 P1 $0.000(15)$

$\mathrm{P} 1 \quad \mathrm{Pd} 1 \quad \mathrm{Pd} 2 \quad \mathrm{P} 3 \quad-13.41(5)$

$\begin{array}{lllll}\mathrm{Pd} 2 & \text { Pd1 } & \text { Sil } & \text { C13 } & 154.96(2)\end{array}$

Si1 Pd1 Pd2 Pd3 179.66(2)

Sil Pd1 Pd2 P2 $\quad$-151.84(2)

C1 Pd1 Pd2 Pd3 $-50.02(6)$

$\begin{array}{lllll}\mathrm{C} 1 & \mathrm{Pd} 1 & \mathrm{Pd} 2 & \mathrm{P} 2 & -21.52(7)\end{array}$

P1 Pd1 Si1 C11 72.02(2)

P1 Pd1 Sil C19 $-46.28(3)$

Sil Pd1 P1 C12 -29.17(3)

C1 Pd1 Si1 C11 $-100.06(5)$

C1 Pd1 Sil C19 141.64(6)

Pd1 Pd2 Pd3 Si3 178.29(3)

Pd1 Pd2 P1 Pd1 $0.000(11)$

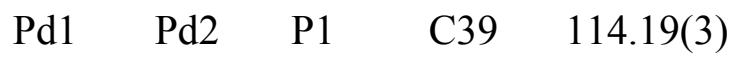

atom1 atom2 atom3 atom4 angle

$\begin{array}{lllll}\mathrm{Pd} 2 & \mathrm{Pd} 1 & \mathrm{P} 1 & \mathrm{C} 12 & 118.42(3)\end{array}$

$\begin{array}{lllll}\mathrm{P} 1 & \mathrm{Pd} 1 & \mathrm{Pd} 2 & \mathrm{Pd} 3 & 135.59(2)\end{array}$

P1 Pd1 Pd2 P2 164.10(3)

$\begin{array}{lllll}\mathrm{Pd} 2 & \mathrm{Pd} 1 & \mathrm{Si} 1 & \mathrm{C} 11 & 39.43(3)\end{array}$

$\begin{array}{lllll}\mathrm{Pd} 2 & \mathrm{Pd} 1 & \mathrm{Si} 1 & \mathrm{C} 19 & -78.87(3)\end{array}$

Si1 Pd1 Pd2 P1 44.06(2)

Si1 Pd1 Pd2 P3 30.66(6)

C1 Pd1 Pd2 P1 174.39(7)

$\begin{array}{lllll}\mathrm{C} 1 & \mathrm{Pd} 1 & \mathrm{Pd} 2 & \mathrm{P} 3 & 160.98(7)\end{array}$

P1 Pd1 Si1 C13 $-172.44(2)$

Si1 Pd1 P1 Pd2 $\quad$-147.587(19)

Sil Pd1 P1 C39 101.13(3)

$\begin{array}{lllll}\text { C1 } & \text { Pd1 } & \text { Si1 } & \text { C13 } & 15.48(6)\end{array}$

$\mathrm{Pd} 1 \quad \mathrm{Pd} 2 \quad \mathrm{Pd} 3 \quad \mathrm{P} 2 \quad 141.646(18)$

Pd1 Pd2 Pd3 C6 $-40.50(2)$

Pd1 Pd2 P1 C12 -120.94(3)

$\begin{array}{lllll}\mathrm{Pd} 1 & \mathrm{Pd} 2 & \mathrm{P} 2 & \mathrm{Pd} 3 & -33.476(17)\end{array}$ 


\begin{tabular}{|c|c|c|c|c|c|c|c|c|c|}
\hline Pd1 & $\operatorname{Pd} 2$ & $\mathrm{P} 2$ & C38 & $-152.24(2)$ & Pd1 & $\mathrm{Pd} 2$ & $\mathrm{P} 2$ & C69 & $77.73(3)$ \\
\hline $\mathrm{Pd} 1$ & $\operatorname{Pd} 2$ & P3 & $\mathrm{C} 75$ & $-135.93(5)$ & Pd1 & $\mathrm{Pd} 2$ & P3 & $\mathrm{C} 81$ & $103.89(6)$ \\
\hline Pd1 & $\mathrm{Pd} 2$ & P3 & $\mathrm{C} 87$ & $-11.50(7)$ & $\mathrm{Pd} 3$ & $\mathrm{Pd} 2$ & $\mathrm{P} 1$ & $\mathrm{Pd} 1$ & $-38.105(17)$ \\
\hline $\mathrm{Pd} 3$ & $\operatorname{Pd} 2$ & $\mathrm{P} 1$ & $\mathrm{C} 12$ & $-159.05(2)$ & $\mathrm{Pd} 3$ & $\mathrm{Pd} 2$ & $\mathrm{P} 1$ & C39 & $76.09(3)$ \\
\hline $\mathrm{P} 1$ & $\mathrm{Pd} 2$ & $\mathrm{Pd} 3$ & $\mathrm{P} 2$ & $173.96(2)$ & $\mathrm{P} 1$ & $\mathrm{Pd} 2$ & $\mathrm{Pd} 3$ & $\mathrm{Si} 3$ & $-149.40(2)$ \\
\hline P1 & $\mathrm{Pd} 2$ & $\mathrm{Pd} 3$ & C6 & $-8.19(3)$ & $\mathrm{Pd} 3$ & $\mathrm{Pd} 2$ & $\mathrm{P} 2$ & $\mathrm{Pd} 3$ & $-0.000(13)$ \\
\hline $\mathrm{Pd} 3$ & $\mathrm{Pd} 2$ & $\mathrm{P} 2$ & C38 & $-118.76(3)$ & $\mathrm{Pd} 3$ & $\operatorname{Pd} 2$ & $\mathrm{P} 2$ & C69 & $111.20(4)$ \\
\hline $\mathrm{P} 2$ & $\mathrm{Pd} 2$ & $\mathrm{Pd} 3$ & $\mathrm{P} 2$ & $0.000(17)$ & $\mathrm{P} 2$ & $\mathrm{Pd} 2$ & $\mathrm{Pd} 3$ & $\mathrm{Si} 3$ & $36.64(3)$ \\
\hline $\mathrm{P} 2$ & $\mathrm{Pd} 2$ & $\mathrm{Pd} 3$ & C6 & $177.85(3)$ & $\mathrm{Pd} 3$ & $\mathrm{Pd} 2$ & P3 & $\mathrm{C} 75$ & $87.47(4)$ \\
\hline $\mathrm{Pd} 3$ & $\mathrm{Pd} 2$ & P3 & $\mathrm{C} 81$ & $-32.70(4)$ & $\mathrm{Pd} 3$ & $\operatorname{Pd} 2$ & P3 & $\mathrm{C} 87$ & $-148.10(2)$ \\
\hline P3 & $\mathrm{Pd} 2$ & $\mathrm{Pd} 3$ & $\mathrm{P} 2$ & $-57.56(3)$ & P3 & $\mathrm{Pd} 2$ & $\mathrm{Pd} 3$ & $\mathrm{Si} 3$ & $-20.92(4)$ \\
\hline P3 & $\mathrm{Pd} 2$ & $\mathrm{Pd} 3$ & C6 & $120.29(3)$ & $\mathrm{P} 1$ & $\mathrm{Pd} 2$ & $\mathrm{P} 2$ & $\mathrm{Pd} 3$ & $-11.06(5)$ \\
\hline P1 & $\mathrm{Pd} 2$ & $\mathrm{P} 2$ & C38 & $-129.82(5)$ & $\mathrm{P} 1$ & $\mathrm{Pd} 2$ & $\mathrm{P} 2$ & C69 & $100.15(5)$ \\
\hline P2 & $\mathrm{Pd} 2$ & $\mathrm{P} 1$ & $\mathrm{Pd} 1$ & $-29.69(5)$ & $\mathrm{P} 2$ & $\mathrm{Pd} 2$ & $\mathrm{P} 1$ & $\mathrm{C} 12$ & $-150.63(4)$ \\
\hline P2 & $\mathrm{Pd} 2$ & $\mathrm{P} 1$ & C39 & $84.50(5)$ & $\mathrm{P} 1$ & $\mathrm{Pd} 2$ & $\mathrm{P} 3$ & $\mathrm{C} 75$ & $-146.47(3)$ \\
\hline P1 & $\mathrm{Pd} 2$ & P3 & C81 & $93.35(3)$ & $\mathrm{P} 1$ & $\mathrm{Pd} 2$ & P3 & $\mathrm{C} 87$ & $-22.04(3)$ \\
\hline P3 & $\mathrm{Pd} 2$ & $\mathrm{P} 1$ & $\mathrm{Pd} 1$ & $174.20(2)$ & P3 & $\mathrm{Pd} 2$ & $\mathrm{P} 1$ & $\mathrm{C} 12$ & $53.25(3)$ \\
\hline P3 & $\mathrm{Pd} 2$ & $\mathrm{P} 1$ & C39 & $-71.61(3)$ & $\mathrm{P} 2$ & $\mathrm{Pd} 2$ & P3 & $\mathrm{C} 75$ & $46.58(3)$ \\
\hline P2 & $\mathrm{Pd} 2$ & P3 & $\mathrm{C} 81$ & $-73.59(3)$ & $\mathrm{P} 2$ & $\mathrm{Pd} 2$ & $\mathrm{P} 3$ & $\mathrm{C} 87$ & $171.01(2)$ \\
\hline P3 & $\mathrm{Pd} 2$ & $\mathrm{P} 2$ & $\mathrm{Pd} 3$ & $145.45(2)$ & $\mathrm{P} 3$ & $\mathrm{Pd} 2$ & $\mathrm{P} 2$ & $\mathrm{C} 38$ & $26.69(3)$ \\
\hline P3 & $\mathrm{Pd} 2$ & $\mathrm{P} 2$ & C69 & $-103.34(3)$ & $\mathrm{Pd} 2$ & $\mathrm{Pd} 3$ & $\mathrm{P} 2$ & $\mathrm{Pd} 2$ & $0.000(12)$ \\
\hline $\mathrm{Pd} 2$ & $\mathrm{Pd} 3$ & $\mathrm{P} 2$ & C38 & $113.74(3)$ & $\mathrm{Pd} 2$ & $\mathrm{Pd} 3$ & $\mathrm{P} 2$ & C69 & $-117.90(3)$ \\
\hline $\operatorname{Pd} 2$ & $\mathrm{Pd} 3$ & $\mathrm{Si} 3$ & $\mathrm{C} 37$ & $-45.46(4)$ & $\mathrm{Pd} 2$ & $\mathrm{Pd} 3$ & $\mathrm{Si} 3$ & $\mathrm{C} 45$ & $-167.94(2)$ \\
\hline $\operatorname{Pd} 2$ & $\mathrm{Pd} 3$ & $\mathrm{Si} 3$ & C51 & $75.45(3)$ & $\mathrm{P} 2$ & $\mathrm{Pd} 3$ & $\mathrm{Si} 3$ & $\mathrm{C} 37$ & $-17.88(3)$ \\
\hline $\mathrm{P} 2$ & $\mathrm{Pd} 3$ & $\mathrm{Si} 3$ & $\mathrm{C} 45$ & $-140.36(2)$ & $\mathrm{P} 2$ & $\mathrm{Pd} 3$ & $\mathrm{Si} 3$ & $\mathrm{C} 51$ & $103.03(3)$ \\
\hline $\mathrm{Si} 3$ & $\mathrm{Pd} 3$ & $\mathrm{P} 2$ & $\operatorname{Pd} 2$ & $-154.759(18)$ & $\mathrm{Si} 3$ & $\mathrm{Pd} 3$ & $\mathrm{P} 2$ & $\mathrm{C} 38$ & $-41.02(3)$ \\
\hline $\mathrm{Si} 3$ & $\mathrm{Pd} 3$ & $\mathrm{P} 2$ & C69 & $87.34(3)$ & $\mathrm{C} 6$ & $\mathrm{Pd} 3$ & $\mathrm{Si} 3$ & $\mathrm{C} 37$ & $166.55(5)$ \\
\hline C6 & $\mathrm{Pd} 3$ & $\mathrm{Si} 3$ & $\mathrm{C} 45$ & $44.07(5)$ & $\mathrm{C} 6$ & $\mathrm{Pd} 3$ & $\mathrm{Si} 3$ & $\mathrm{C} 51$ & $-72.54(5)$ \\
\hline Pd1 & $\mathrm{P} 1$ & $\mathrm{C} 12$ & $\mathrm{Si} 2$ & $-29.51(11)$ & $\mathrm{Pd} 1$ & $\mathrm{P} 1$ & C39 & $\mathrm{C} 40$ & $32.12(13)$ \\
\hline Pd1 & $\mathrm{P} 1$ & C39 & $\mathrm{C} 44$ & $-155.14(9)$ & $\mathrm{Pd} 2$ & $\mathrm{P} 1$ & $\mathrm{C} 12$ & $\mathrm{Si} 2$ & $67.18(10)$ \\
\hline $\operatorname{Pd} 2$ & $\mathrm{P} 1$ & C39 & $\mathrm{C} 40$ & $-58.12(12)$ & $\mathrm{Pd} 2$ & $\mathrm{P} 1$ & C39 & $\mathrm{C} 44$ & $114.62(11)$ \\
\hline $\mathrm{C} 12$ & $\mathrm{P} 1$ & C39 & $\mathrm{C} 40$ & $169.83(10)$ & $\mathrm{C} 12$ & $\mathrm{P} 1$ & C39 & $\mathrm{C} 44$ & $-17.44(14)$ \\
\hline C39 & $\mathrm{P} 1$ & $\mathrm{C} 12$ & $\mathrm{Si} 2$ & $-164.20(8)$ & $\mathrm{Pd} 2$ & $\mathrm{P} 2$ & C38 & $\mathrm{Si} 4$ & $161.28(6)$ \\
\hline $\mathrm{Pd} 2$ & $\mathrm{P} 2$ & C69 & $\mathrm{C} 70$ & $-59.07(14)$ & $\mathrm{Pd} 2$ & $\mathrm{P} 2$ & C69 & $\mathrm{C} 74$ & $118.25(12)$ \\
\hline $\mathrm{Pd} 3$ & $\mathrm{P} 2$ & $\mathrm{C} 38$ & $\mathrm{Si} 4$ & $68.05(10)$ & $\mathrm{Pd} 3$ & $\mathrm{P} 2$ & C69 & $\mathrm{C} 70$ & $32.56(14)$ \\
\hline $\mathrm{Pd} 3$ & $\mathrm{P} 2$ & C69 & C74 & $-150.13(11)$ & $\mathrm{C} 38$ & $\mathrm{P} 2$ & C69 & $\mathrm{C} 70$ & $167.32(12)$ \\
\hline $\mathrm{C} 38$ & $\mathrm{P} 2$ & C69 & $\mathrm{C} 74$ & $-15.36(16)$ & C69 & $\mathrm{P} 2$ & $\mathrm{C} 38$ & $\mathrm{Si} 4$ & $-63.00(12)$ \\
\hline $\mathrm{Pd} 2$ & P3 & $\mathrm{C} 75$ & $\mathrm{C} 76$ & 178.97(9) & $\mathrm{Pd} 2$ & P3 & $\mathrm{C} 75$ & $\mathrm{C} 80$ & $-1.04(16)$ \\
\hline $\mathrm{Pd} 2$ & P3 & $\mathrm{C} 81$ & C82 & $-39.73(12)$ & $\mathrm{Pd} 2$ & P3 & $\mathrm{C} 81$ & $\mathrm{C} 86$ & $140.60(11)$ \\
\hline
\end{tabular}




\begin{tabular}{|c|c|c|c|c|c|c|c|c|c|}
\hline $\operatorname{Pd} 2$ & $\mathrm{P} 3$ & $\mathrm{C} 87$ & $\mathrm{C} 88$ & $82.87(12)$ & $\operatorname{Pd} 2$ & P3 & $\mathrm{C} 87$ & C92 & $-89.94(11)$ \\
\hline $\mathrm{C} 75$ & P3 & $\mathrm{C} 81$ & $\mathrm{C} 82$ & $-168.72(11)$ & $\mathrm{C} 75$ & P3 & $\mathrm{C} 81$ & C86 & $11.62(16)$ \\
\hline C81 & P3 & $\mathrm{C} 75$ & C76 & $-60.06(15)$ & $\mathrm{C} 81$ & P3 & $\mathrm{C} 75$ & $\mathrm{C} 80$ & 119.93(12) \\
\hline $\mathrm{C} 75$ & $\mathrm{P} 3$ & $\mathrm{C} 87$ & $\mathrm{C} 88$ & $-143.95(11)$ & $\mathrm{C} 75$ & P3 & $\mathrm{C} 87$ & C92 & $43.24(13)$ \\
\hline $\mathrm{C} 87$ & P3 & $\mathrm{C} 75$ & C76 & $47.13(15)$ & $\mathrm{C} 87$ & P3 & $\mathrm{C} 75$ & $\mathrm{C} 80$ & $-132.88(13)$ \\
\hline C81 & P3 & $\mathrm{C} 87$ & $\mathrm{C} 88$ & $-34.76(13)$ & $\mathrm{C} 81$ & P3 & $\mathrm{C} 87$ & C92 & $152.42(11)$ \\
\hline C87 & P3 & $\mathrm{C} 81$ & $\mathrm{C} 82$ & $85.14(12)$ & $\mathrm{C} 87$ & P3 & $\mathrm{C} 81$ & $\mathrm{C} 86$ & $-94.53(14)$ \\
\hline $\operatorname{Pd} 1$ & Sil & $\mathrm{C} 11$ & $\mathrm{Si} 2$ & $-61.16(10)$ & $\mathrm{Pd} 1$ & Sil & $\mathrm{C} 13$ & $\mathrm{C} 14$ & $88.67(12)$ \\
\hline $\operatorname{Pd} 1$ & Sil & $\mathrm{C} 13$ & $\mathrm{C} 18$ & $-92.54(13)$ & $\mathrm{Pd} 1$ & Sil & $\mathrm{C} 19$ & $\mathrm{C} 20$ & $-34.85(14)$ \\
\hline Pd1 & Sil & $\mathrm{C} 19$ & $\mathrm{C} 24$ & $143.15(9)$ & $\mathrm{C} 11$ & Sil & $\mathrm{C} 13$ & $\mathrm{C} 14$ & $-155.55(12)$ \\
\hline $\mathrm{C} 11$ & Sil & $\mathrm{C} 13$ & $\mathrm{C} 18$ & $23.23(14)$ & $\mathrm{C} 13$ & Sil & $\mathrm{C} 11$ & $\mathrm{Si} 2$ & $176.39(9)$ \\
\hline $\mathrm{C} 11$ & Sil & $\mathrm{C} 19$ & $\mathrm{C} 20$ & $-153.23(11)$ & $\mathrm{C} 11$ & Sil & $\mathrm{C} 19$ & $\mathrm{C} 24$ & $24.77(13)$ \\
\hline C19 & Sil & $\mathrm{C} 11$ & $\mathrm{Si} 2$ & $66.33(11)$ & $\mathrm{C} 13$ & Sil & $\mathrm{C} 19$ & $\mathrm{C} 20$ & $96.30(13)$ \\
\hline C13 & Sil & $\mathrm{C} 19$ & $\mathrm{C} 24$ & $-85.70(13)$ & C19 & Sil & $\mathrm{C} 13$ & $\mathrm{C} 14$ & $-44.89(15)$ \\
\hline C19 & Sil & $\mathrm{C} 13$ & $\mathrm{C} 18$ & $133.90(13)$ & $\mathrm{C} 11$ & $\mathrm{Si} 2$ & $\mathrm{C} 12$ & $\mathrm{P} 1$ & $54.66(11)$ \\
\hline $\mathrm{C} 12$ & $\mathrm{Si} 2$ & $\mathrm{C} 11$ & Si1 & $-5.90(12)$ & $\mathrm{C} 11$ & $\mathrm{Si} 2$ & $\mathrm{C} 25$ & $\mathrm{C} 26$ & $-29.83(14)$ \\
\hline C11 & $\mathrm{Si} 2$ & $\mathrm{C} 25$ & $\mathrm{C} 30$ & $149.00(11)$ & $\mathrm{C} 25$ & $\mathrm{Si} 2$ & $\mathrm{C} 11$ & Sil & $-123.36(9)$ \\
\hline $\mathrm{C} 11$ & $\mathrm{Si} 2$ & $\mathrm{C} 31$ & $\mathrm{C} 32$ & $136.23(12)$ & $\mathrm{C} 11$ & $\mathrm{Si} 2$ & $\mathrm{C} 31$ & $\mathrm{C} 36$ & $-42.52(14)$ \\
\hline $\mathrm{C} 31$ & $\mathrm{Si} 2$ & $\mathrm{C} 11$ & Sil & $116.99(10)$ & $\mathrm{C} 12$ & $\mathrm{Si} 2$ & $\mathrm{C} 25$ & $\mathrm{C} 26$ & $-151.14(11)$ \\
\hline $\mathrm{C} 12$ & $\mathrm{Si} 2$ & $\mathrm{C} 25$ & $\mathrm{C} 30$ & $27.70(13)$ & $\mathrm{C} 25$ & $\mathrm{Si} 2$ & $\mathrm{C} 12$ & $\mathrm{P} 1$ & $175.00(8)$ \\
\hline $\mathrm{C} 12$ & $\mathrm{Si} 2$ & $\mathrm{C} 31$ & $\mathrm{C} 32$ & $-100.31(12)$ & $\mathrm{C} 12$ & $\mathrm{Si} 2$ & $\mathrm{C} 31$ & $\mathrm{C} 36$ & $80.94(13)$ \\
\hline C31 & $\mathrm{Si} 2$ & $\mathrm{C} 12$ & $\mathrm{P} 1$ & $-66.77(11)$ & $\mathrm{C} 25$ & $\mathrm{Si} 2$ & $\mathrm{C} 31$ & $\mathrm{C} 32$ & $15.75(14)$ \\
\hline $\mathrm{C} 25$ & $\mathrm{Si} 2$ & $\mathrm{C} 31$ & $\mathrm{C} 36$ & $-163.00(11)$ & $\mathrm{C} 31$ & $\mathrm{Si} 2$ & $\mathrm{C} 25$ & $\mathrm{C} 26$ & $89.39(13)$ \\
\hline C31 & $\mathrm{Si} 2$ & $\mathrm{C} 25$ & $\mathrm{C} 30$ & $-91.77(12)$ & $\mathrm{Pd} 3$ & $\mathrm{Si} 3$ & C37 & $\mathrm{Si} 4$ & $62.20(10)$ \\
\hline $\mathrm{Pd} 3$ & $\mathrm{Si} 3$ & $\mathrm{C} 45$ & $\mathrm{C} 46$ & $-85.60(13)$ & $\mathrm{Pd} 3$ & $\mathrm{Si} 3$ & $\mathrm{C} 45$ & $\mathrm{C} 50$ & $90.07(12)$ \\
\hline $\mathrm{Pd} 3$ & $\mathrm{Si} 3$ & $\mathrm{C} 51$ & $\mathrm{C} 52$ & $-23.34(13)$ & $\mathrm{Pd} 3$ & $\mathrm{Si} 3$ & $\mathrm{C} 51$ & $\mathrm{C} 56$ & 161.99(9) \\
\hline $\mathrm{C} 37$ & $\mathrm{Si} 3$ & $\mathrm{C} 45$ & $\mathrm{C} 46$ & $143.00(12)$ & $\mathrm{C} 37$ & $\mathrm{Si} 3$ & $\mathrm{C} 45$ & $\mathrm{C} 50$ & $-41.33(14)$ \\
\hline $\mathrm{C} 45$ & $\mathrm{Si} 3$ & $\mathrm{C} 37$ & $\mathrm{Si} 4$ & $-172.79(9)$ & $\mathrm{C} 37$ & $\mathrm{Si} 3$ & $\mathrm{C} 51$ & $\mathrm{C} 52$ & 107.01(12) \\
\hline $\mathrm{C} 37$ & $\mathrm{Si} 3$ & $\mathrm{C} 51$ & $\mathrm{C} 56$ & $-67.66(13)$ & C51 & $\mathrm{Si} 3$ & C37 & $\mathrm{Si} 4$ & $-60.76(11)$ \\
\hline $\mathrm{C} 45$ & $\mathrm{Si} 3$ & $\mathrm{C} 51$ & $\mathrm{C} 52$ & $-142.22(11)$ & $\mathrm{C} 45$ & $\mathrm{Si} 3$ & $\mathrm{C} 51$ & $\mathrm{C} 56$ & $43.11(13)$ \\
\hline $\mathrm{C} 51$ & $\mathrm{Si} 3$ & $\mathrm{C} 45$ & $\mathrm{C} 46$ & $32.39(14)$ & C51 & $\mathrm{Si} 3$ & $\mathrm{C} 45$ & $\mathrm{C} 50$ & $-151.94(12)$ \\
\hline $\mathrm{C} 37$ & $\mathrm{Si} 4$ & $\mathrm{C} 38$ & $\mathrm{P} 2$ & $-19.06(12)$ & $\mathrm{C} 38$ & $\mathrm{Si} 4$ & C37 & $\mathrm{Si} 3$ & $-40.38(11)$ \\
\hline $\mathrm{C} 37$ & $\mathrm{Si} 4$ & $\mathrm{C} 57$ & $\mathrm{C} 58$ & $-151.04(10)$ & $\mathrm{C} 37$ & $\mathrm{Si} 4$ & $\mathrm{C} 57$ & C62 & $35.31(14)$ \\
\hline C57 & $\mathrm{Si} 4$ & C37 & $\mathrm{Si} 3$ & $80.51(11)$ & $\mathrm{C} 37$ & $\mathrm{Si} 4$ & C63 & C64 & $160.71(11)$ \\
\hline C37 & $\mathrm{Si} 4$ & C63 & C68 & $-15.92(15)$ & C63 & $\mathrm{Si} 4$ & C37 & $\mathrm{Si} 3$ & $-159.37(9)$ \\
\hline $\mathrm{C} 38$ & $\mathrm{Si} 4$ & $\mathrm{C} 57$ & $\mathrm{C} 58$ & $-29.96(13)$ & $\mathrm{C} 38$ & $\mathrm{Si} 4$ & $\mathrm{C} 57$ & C62 & $156.39(11)$ \\
\hline C57 & $\mathrm{Si} 4$ & C38 & $\mathrm{P} 2$ & $-142.53(9)$ & $\mathrm{C} 38$ & $\mathrm{Si} 4$ & C63 & C64 & $40.76(14)$ \\
\hline $\mathrm{C} 38$ & $\mathrm{Si} 4$ & C63 & C68 & $-135.87(12)$ & C63 & $\mathrm{Si} 4$ & $\mathrm{C} 38$ & $\mathrm{P} 2$ & $103.17(10)$ \\
\hline C57 & $\mathrm{Si} 4$ & C63 & C64 & $-75.27(13)$ & C57 & $\mathrm{Si} 4$ & C63 & C68 & 108.09(13) \\
\hline
\end{tabular}




\begin{tabular}{|c|c|c|c|c|c|c|c|c|c|}
\hline C63 & $\mathrm{Si} 4$ & $\mathrm{C} 57$ & $\mathrm{C} 58$ & $85.16(12)$ & $\mathrm{C} 63$ & $\mathrm{Si} 4$ & $\mathrm{C} 57$ & C62 & $-88.49(13)$ \\
\hline $\mathrm{C} 1$ & N1 & C93 & $\mathrm{C} 2$ & $-174.9(5)$ & $\mathrm{C} 1$ & $\mathrm{~N} 1$ & C93 & $\mathrm{C} 3$ & $111.7(11)$ \\
\hline $\mathrm{C} 1$ & N1 & C93 & $\mathrm{C} 5$ & $-92.1(13)$ & $\mathrm{C} 1$ & N1 & C93 & C94 & $-4.6(17)$ \\
\hline $\mathrm{C} 1$ & $\mathrm{~N} 1$ & $\mathrm{C} 93$ & C95 & $-123.2(10)$ & $\mathrm{C} 2$ & $\mathrm{~N} 1$ & C93 & $\mathrm{C} 2$ & $0.0(2)$ \\
\hline $\mathrm{C} 2$ & $\mathrm{~N} 1$ & $\mathrm{C} 93$ & $\mathrm{C} 3$ & $-73.4(9)$ & $\mathrm{C} 2$ & $\mathrm{~N} 1$ & C93 & $\mathrm{C} 5$ & $82.8(10)$ \\
\hline $\mathrm{C} 2$ & $\mathrm{~N} 1$ & $\mathrm{C} 93$ & C94 & $170.2(15)$ & $\mathrm{C} 2$ & $\mathrm{~N} 1$ & C93 & C95 & $51.6(7)$ \\
\hline C93 & $\mathrm{N} 1$ & $\mathrm{C} 2$ & $\mathrm{C} 3$ & $67.4(9)$ & C93 & $\mathrm{N} 1$ & $\mathrm{C} 2$ & $\mathrm{C} 4$ & $-174.5(9)$ \\
\hline C93 & $\mathrm{N} 1$ & $\mathrm{C} 2$ & $\mathrm{C} 5$ & $-56.4(9)$ & $\mathrm{C} 93$ & $\mathrm{~N} 1$ & $\mathrm{C} 2$ & C93 & $0.0(9)$ \\
\hline C93 & $\mathrm{N} 1$ & $\mathrm{C} 2$ & C95 & $-85.7(9)$ & $\mathrm{N} 1$ & $\mathrm{C} 2$ & $\mathrm{C} 3$ & C93 & $-75.9(2)$ \\
\hline N1 & $\mathrm{C} 2$ & $\mathrm{C} 4$ & C95 & 117.1(2) & N1 & $\mathrm{C} 2$ & $\mathrm{C} 5$ & C93 & $72.0(2)$ \\
\hline N1 & $\mathrm{C} 2$ & $\mathrm{C} 5$ & C94 & $73.5(2)$ & N1 & $\mathrm{C} 2$ & $\mathrm{C} 5$ & C95 & $-119.6(3)$ \\
\hline N1 & $\mathrm{C} 2$ & $\mathrm{C} 93$ & N1 & $0.00(6)$ & N1 & $\mathrm{C} 2$ & C93 & $\mathrm{C} 3$ & $111.5(4)$ \\
\hline N1 & $\mathrm{C} 2$ & C93 & $\mathrm{C} 5$ & $-113.2(6)$ & N1 & $\mathrm{C} 2$ & C93 & C95 & $-118.9(2)$ \\
\hline N1 & $\mathrm{C} 2$ & C95 & $\mathrm{C} 4$ & $-105.3(6)$ & N1 & $\mathrm{C} 2$ & C95 & $\mathrm{C} 5$ & $72(2)$ \\
\hline N1 & $\mathrm{C} 2$ & C95 & C93 & $81.9(7)$ & $\mathrm{C} 3$ & $\mathrm{C} 2$ & $\mathrm{C} 4$ & C95 & $-125.9(2)$ \\
\hline $\mathrm{C} 4$ & $\mathrm{C} 2$ & $\mathrm{C} 3$ & C93 & $167.0(3)$ & $\mathrm{C} 3$ & $\mathrm{C} 2$ & $\mathrm{C} 5$ & C93 & $-47.2(2)$ \\
\hline $\mathrm{C} 3$ & $\mathrm{C} 2$ & $\mathrm{C} 5$ & C94 & $-45.7(2)$ & $\mathrm{C} 3$ & $\mathrm{C} 2$ & $\mathrm{C} 5$ & C95 & $121.2(3)$ \\
\hline $\mathrm{C} 5$ & $\mathrm{C} 2$ & $\mathrm{C} 3$ & C93 & $42.5(3)$ & $\mathrm{C} 3$ & $\mathrm{C} 2$ & C93 & N1 & $-111.5(4)$ \\
\hline $\mathrm{C} 3$ & $\mathrm{C} 2$ & C93 & $\mathrm{C} 3$ & $0.00(10)$ & $\mathrm{C} 3$ & $\mathrm{C} 2$ & C93 & $\mathrm{C} 5$ & $135.3(7)$ \\
\hline $\mathrm{C} 3$ & $\mathrm{C} 2$ & $\mathrm{C} 93$ & C95 & $129.6(3)$ & C93 & $\mathrm{C} 2$ & $\mathrm{C} 3$ & C93 & $0.0(9)$ \\
\hline $\mathrm{C} 3$ & $\mathrm{C} 2$ & C95 & $\mathrm{C} 4$ & $107.2(8)$ & $\mathrm{C} 3$ & $\mathrm{C} 2$ & C95 & $\mathrm{C} 5$ & $-76(2)$ \\
\hline $\mathrm{C} 3$ & $\mathrm{C} 2$ & C95 & C93 & $-65.6(9)$ & C95 & $\mathrm{C} 2$ & $\mathrm{C} 3$ & C93 & $74.4(14)$ \\
\hline $\mathrm{C} 4$ & $\mathrm{C} 2$ & $\mathrm{C} 5$ & C93 & $-171.4(3)$ & $\mathrm{C} 4$ & $\mathrm{C} 2$ & $\mathrm{C} 5$ & C94 & $-169.88(19)$ \\
\hline $\mathrm{C} 4$ & $\mathrm{C} 2$ & $\mathrm{C} 5$ & C95 & $-2.9(3)$ & $\mathrm{C} 5$ & $\mathrm{C} 2$ & $\mathrm{C} 4$ & $\mathrm{C} 95$ & $1.48(17)$ \\
\hline $\mathrm{C} 4$ & $\mathrm{C} 2$ & C95 & $\mathrm{C} 4$ & $0.00(12)$ & $\mathrm{C} 4$ & $\mathrm{C} 2$ & C95 & $\mathrm{C} 5$ & $177(2)$ \\
\hline $\mathrm{C} 4$ & $\mathrm{C} 2$ & C95 & C93 & $-172.8(3)$ & C95 & $\mathrm{C} 2$ & $\mathrm{C} 4$ & C95 & $0.0(11)$ \\
\hline C5 & $\mathrm{C} 2$ & C93 & N1 & $113.2(6)$ & $\mathrm{C} 5$ & $\mathrm{C} 2$ & C93 & $\mathrm{C} 3$ & $-135.3(8)$ \\
\hline C5 & $\mathrm{C} 2$ & C93 & $\mathrm{C} 5$ & $0.00(16)$ & $\mathrm{C} 5$ & $\mathrm{C} 2$ & C93 & C95 & $-5.7(5)$ \\
\hline C93 & $\mathrm{C} 2$ & $\mathrm{C} 5$ & C93 & $0.0(10)$ & C93 & $\mathrm{C} 2$ & $\mathrm{C} 5$ & C94 & $1.5(10)$ \\
\hline C93 & $\mathrm{C} 2$ & $\mathrm{C} 5$ & C95 & $168.5(11)$ & $\mathrm{C} 5$ & $\mathrm{C} 2$ & C95 & $\mathrm{C} 4$ & $-177(2)$ \\
\hline $\mathrm{C} 5$ & $\mathrm{C} 2$ & C95 & $\mathrm{C} 5$ & $-0.0(3)$ & $\mathrm{C} 5$ & $\mathrm{C} 2$ & C95 & C93 & $10(2)$ \\
\hline C95 & $\mathrm{C} 2$ & $\mathrm{C} 5$ & C93 & $-168(2)$ & C95 & $\mathrm{C} 2$ & $\mathrm{C} 5$ & C94 & $-167(2)$ \\
\hline C95 & $\mathrm{C} 2$ & $\mathrm{C} 5$ & C95 & $-0(2)$ & C93 & $\mathrm{C} 2$ & C95 & $\mathrm{C} 4$ & $172.8(9)$ \\
\hline C93 & $\mathrm{C} 2$ & C95 & $\mathrm{C} 5$ & $-10(2)$ & C93 & $\mathrm{C} 2$ & C95 & C93 & $-0.0(9)$ \\
\hline C95 & $\mathrm{C} 2$ & C93 & N1 & $118.9(11)$ & C95 & $\mathrm{C} 2$ & C93 & $\mathrm{C} 3$ & $-129.6(12)$ \\
\hline C95 & $\mathrm{C} 2$ & C93 & $\mathrm{C} 5$ & $5.7(12)$ & C95 & $\mathrm{C} 2$ & C93 & C95 & $-0.0(11)$ \\
\hline $\mathrm{C} 2$ & $\mathrm{C} 3$ & $\mathrm{C} 93$ & N1 & $65.2(9)$ & $\mathrm{C} 2$ & $\mathrm{C} 3$ & C93 & $\mathrm{C} 2$ & $0.00(18)$ \\
\hline $\mathrm{C} 2$ & $\mathrm{C} 3$ & C93 & $\mathrm{C} 5$ & $-83.0(14)$ & $\mathrm{C} 2$ & $\mathrm{C} 3$ & C93 & C94 & $-171.2(14)$ \\
\hline $\mathrm{C} 2$ & $\mathrm{C} 3$ & $\mathrm{C} 93$ & C95 & $-49.8(7)$ & $\mathrm{C} 2$ & $\mathrm{C} 4$ & C95 & $\mathrm{C} 2$ & $0.00(13)$ \\
\hline
\end{tabular}




\begin{tabular}{|c|c|c|c|c|c|c|c|c|c|}
\hline $\mathrm{C} 2$ & $\mathrm{C} 4$ & C95 & $\mathrm{C} 5$ & $-6(4)$ & $\mathrm{C} 2$ & $\mathrm{C} 4$ & C95 & C93 & $3.57(18)$ \\
\hline $\mathrm{C} 2$ & $\mathrm{C} 5$ & C93 & N1 & $-68.2(10)$ & $\mathrm{C} 2$ & $\mathrm{C} 5$ & C93 & $\mathrm{C} 2$ & $-0.00(19)$ \\
\hline $\mathrm{C} 2$ & $\mathrm{C} 5$ & C93 & $\mathrm{C} 3$ & $78.7(14)$ & $\mathrm{C} 2$ & $\mathrm{C} 5$ & C93 & C94 & $178.2(12)$ \\
\hline${ }^{2}$ & $\mathrm{C} 5$ & $\mathrm{C} 93$ & C95 & $9.6(9)$ & $\mathrm{C} 2$ & $\mathrm{C} 5$ & C94 & C93 & $-0.9(3)$ \\
\hline $\mathrm{C} 2$ & $\mathrm{C} 5$ & C95 & $\mathrm{C} 2$ & $-0.00(11)$ & $\mathrm{C} 2$ & $\mathrm{C} 5$ & C95 & $\mathrm{C} 4$ & $5(4)$ \\
\hline $\mathrm{C} 2$ & $\mathrm{C} 5$ & C95 & C93 & $-5.5(11)$ & C93 & $\mathrm{C} 5$ & C94 & C93 & $-0.0(6)$ \\
\hline C94 & $\mathrm{C} 5$ & C93 & N1 & $113.6(10)$ & C94 & $\mathrm{C} 5$ & C93 & $\mathrm{C} 2$ & $-178.2(13)$ \\
\hline C94 & $\mathrm{C} 5$ & C93 & $\mathrm{C} 3$ & $-99.4(15)$ & C94 & $\mathrm{C} 5$ & C93 & C94 & $-0.0(5)$ \\
\hline C94 & $\mathrm{C} 5$ & $\mathrm{C} 93$ & C95 & $-168.5(7)$ & $\mathrm{C} 93$ & $\mathrm{C} 5$ & C95 & $\mathrm{C} 2$ & $5.5(12)$ \\
\hline C93 & $\mathrm{C} 5$ & C95 & $\mathrm{C} 4$ & $10(5)$ & C93 & $\mathrm{C} 5$ & C95 & C93 & $-0.0(5)$ \\
\hline C95 & $\mathrm{C} 5$ & C93 & N1 & $-78(2)$ & C95 & $\mathrm{C} 5$ & C93 & $\mathrm{C} 2$ & $-10(2)$ \\
\hline C95 & $\mathrm{C} 5$ & $\mathrm{C} 93$ & $\mathrm{C} 3$ & $69(2)$ & C95 & $\mathrm{C} 5$ & C93 & C94 & $169(2)$ \\
\hline C95 & $\mathrm{C} 5$ & C93 & C95 & $-0.0(19)$ & C94 & $\mathrm{C} 5$ & C95 & $\mathrm{C} 2$ & $19(3)$ \\
\hline C94 & $\mathrm{C} 5$ & C95 & $\mathrm{C} 4$ & $24(7)$ & C94 & $\mathrm{C} 5$ & C95 & C93 & $14(2)$ \\
\hline C95 & $\mathrm{C} 5$ & C94 & C93 & $-17(3)$ & Sil & $\mathrm{C} 13$ & $\mathrm{C} 14$ & $\mathrm{C} 15$ & 178.91(11) \\
\hline Sil & $\mathrm{C} 13$ & $\mathrm{C} 18$ & $\mathrm{C} 17$ & $-178.21(12)$ & $\mathrm{C} 14$ & $\mathrm{C} 13$ & $\mathrm{C} 18$ & $\mathrm{C} 17$ & $0.6(3)$ \\
\hline $\mathrm{C} 18$ & $\mathrm{C} 13$ & $\mathrm{C} 14$ & $\mathrm{C} 15$ & $0.1(3)$ & $\mathrm{C} 13$ & $\mathrm{C} 14$ & $\mathrm{C} 15$ & $\mathrm{C} 16$ & $-0.8(3)$ \\
\hline $\mathrm{C} 14$ & $\mathrm{C} 15$ & $\mathrm{C} 16$ & $\mathrm{C} 17$ & $0.9(3)$ & $\mathrm{C} 15$ & $\mathrm{C} 16$ & $\mathrm{C} 17$ & $\mathrm{C} 18$ & $-0.2(3)$ \\
\hline $\mathrm{C} 16$ & $\mathrm{C} 17$ & $\mathrm{C} 18$ & $\mathrm{C} 13$ & $-0.6(3)$ & Sil & C19 & $\mathrm{C} 20$ & $\mathrm{C} 21$ & $179.89(11)$ \\
\hline Si1 & C19 & $\mathrm{C} 24$ & $\mathrm{C} 23$ & $-179.80(11)$ & $\mathrm{C} 20$ & C19 & $\mathrm{C} 24$ & $\mathrm{C} 23$ & $-1.7(3)$ \\
\hline $\mathrm{C} 24$ & $\mathrm{C} 19$ & $\mathrm{C} 20$ & $\mathrm{C} 21$ & $1.8(3)$ & C19 & $\mathrm{C} 20$ & $\mathrm{C} 21$ & $\mathrm{C} 22$ & $-0.4(3)$ \\
\hline $\mathrm{C} 20$ & $\mathrm{C} 21$ & $\mathrm{C} 22$ & $\mathrm{C} 23$ & $-1.3(3)$ & $\mathrm{C} 21$ & $\mathrm{C} 22$ & $\mathrm{C} 23$ & $\mathrm{C} 24$ & $1.4(3)$ \\
\hline $\mathrm{C} 22$ & $\mathrm{C} 23$ & $\mathrm{C} 24$ & $\mathrm{C} 19$ & $0.1(3)$ & $\mathrm{Si} 2$ & $\mathrm{C} 25$ & $\mathrm{C} 26$ & $\mathrm{C} 27$ & $178.95(11)$ \\
\hline $\mathrm{Si} 2$ & $\mathrm{C} 25$ & $\mathrm{C} 30$ & $\mathrm{C} 29$ & $-179.57(11)$ & $\mathrm{C} 26$ & $\mathrm{C} 25$ & $\mathrm{C} 30$ & $\mathrm{C} 29$ & $-0.7(2)$ \\
\hline $\mathrm{C} 30$ & $\mathrm{C} 25$ & $\mathrm{C} 26$ & $\mathrm{C} 27$ & $0.1(2)$ & $\mathrm{C} 25$ & $\mathrm{C} 26$ & $\mathrm{C} 27$ & $\mathrm{C} 28$ & $0.6(3)$ \\
\hline $\mathrm{C} 26$ & $\mathrm{C} 27$ & $\mathrm{C} 28$ & $\mathrm{C} 29$ & $-0.7(3)$ & $\mathrm{C} 27$ & $\mathrm{C} 28$ & $\mathrm{C} 29$ & $\mathrm{C} 30$ & $0.1(3)$ \\
\hline $\mathrm{C} 28$ & $\mathrm{C} 29$ & $\mathrm{C} 30$ & $\mathrm{C} 25$ & $0.6(3)$ & $\mathrm{Si} 2$ & $\mathrm{C} 31$ & $\mathrm{C} 32$ & $\mathrm{C} 33$ & $-179.07(11)$ \\
\hline $\mathrm{Si} 2$ & $\mathrm{C} 31$ & $\mathrm{C} 36$ & $\mathrm{C} 35$ & $178.69(11)$ & $\mathrm{C} 32$ & $\mathrm{C} 31$ & $\mathrm{C} 36$ & $\mathrm{C} 35$ & $-0.1(2)$ \\
\hline $\mathrm{C} 36$ & $\mathrm{C} 31$ & $\mathrm{C} 32$ & $\mathrm{C} 33$ & $-0.3(2)$ & $\mathrm{C} 31$ & $\mathrm{C} 32$ & $\mathrm{C} 33$ & C34 & $0.3(3)$ \\
\hline $\mathrm{C} 32$ & C33 & $\mathrm{C} 34$ & $\mathrm{C} 35$ & $0.1(3)$ & $\mathrm{C} 33$ & $\mathrm{C} 34$ & $\mathrm{C} 35$ & $\mathrm{C} 36$ & $-0.5(3)$ \\
\hline C34 & $\mathrm{C} 35$ & $\mathrm{C} 36$ & $\mathrm{C} 31$ & $0.5(3)$ & $\mathrm{P} 1$ & C39 & $\mathrm{C} 40$ & $\mathrm{C} 41$ & $171.78(11)$ \\
\hline $\mathrm{P} 1$ & C39 & $\mathrm{C} 44$ & $\mathrm{C} 43$ & $-170.95(11)$ & $\mathrm{C} 40$ & C39 & $\mathrm{C} 44$ & $\mathrm{C} 43$ & $1.8(2)$ \\
\hline $\mathrm{C} 44$ & C39 & $\mathrm{C} 40$ & $\mathrm{C} 41$ & $-1.3(2)$ & C39 & $\mathrm{C} 40$ & $\mathrm{C} 41$ & $\mathrm{C} 42$ & $-0.4(3)$ \\
\hline $\mathrm{C} 40$ & $\mathrm{C} 41$ & $\mathrm{C} 42$ & $\mathrm{C} 43$ & $1.7(3)$ & $\mathrm{C} 41$ & $\mathrm{C} 42$ & $\mathrm{C} 43$ & $\mathrm{C} 44$ & $-1.2(3)$ \\
\hline $\mathrm{C} 42$ & $\mathrm{C} 43$ & $\mathrm{C} 44$ & C39 & $-0.5(3)$ & $\mathrm{Si} 3$ & $\mathrm{C} 45$ & $\mathrm{C} 46$ & $\mathrm{C} 47$ & $176.58(12)$ \\
\hline $\mathrm{Si} 3$ & $\mathrm{C} 45$ & $\mathrm{C} 50$ & $\mathrm{C} 49$ & $-175.46(12)$ & $\mathrm{C} 46$ & $\mathrm{C} 45$ & $\mathrm{C} 50$ & $\mathrm{C} 49$ & $0.4(3)$ \\
\hline $\mathrm{C} 50$ & $\mathrm{C} 45$ & $\mathrm{C} 46$ & $\mathrm{C} 47$ & $0.7(3)$ & $\mathrm{C} 45$ & $\mathrm{C} 46$ & $\mathrm{C} 47$ & $\mathrm{C} 48$ & $-0.8(3)$ \\
\hline $\mathrm{C} 46$ & $\mathrm{C} 47$ & $\mathrm{C} 48$ & $\mathrm{C} 49$ & $-0.1(3)$ & $\mathrm{C} 47$ & $\mathrm{C} 48$ & $\mathrm{C} 49$ & $\mathrm{C} 50$ & $1.2(3)$ \\
\hline $\mathrm{C} 48$ & $\mathrm{C} 49$ & $\mathrm{C} 50$ & $\mathrm{C} 45$ & $-1.3(3)$ & $\mathrm{Si} 3$ & $\mathrm{C} 51$ & $\mathrm{C} 52$ & $\mathrm{C} 53$ & $-174.01(12)$ \\
\hline
\end{tabular}




\begin{tabular}{|c|c|c|c|c|c|c|c|c|c|}
\hline $\mathrm{Si} 3$ & C51 & $\mathrm{C} 56$ & $\mathrm{C} 55$ & $175.27(11)$ & C52 & $\mathrm{C} 51$ & $\mathrm{C} 56$ & C55 & $0.4(3)$ \\
\hline $\mathrm{C} 56$ & C51 & $\mathrm{C} 52$ & $\mathrm{C} 53$ & $0.9(3)$ & C51 & $\mathrm{C} 52$ & $\mathrm{C} 53$ & $\mathrm{C} 54$ & $-1.6(3)$ \\
\hline C52 & $\mathrm{C} 53$ & $\mathrm{C} 54$ & $\mathrm{C} 55$ & $0.9(3)$ & $\mathrm{C} 53$ & $\mathrm{C} 54$ & $\mathrm{C} 55$ & $\mathrm{C} 56$ & $0.3(3)$ \\
\hline C54 & C55 & $\mathrm{C} 56$ & $\mathrm{C} 51$ & $-1.0(3)$ & $\mathrm{Si} 4$ & $\mathrm{C} 57$ & $\mathrm{C} 58$ & C59 & $-173.66(11)$ \\
\hline $\mathrm{Si} 4$ & $\mathrm{C} 57$ & C62 & C61 & $175.31(11)$ & $\mathrm{C} 58$ & $\mathrm{C} 57$ & C62 & C61 & $1.4(2)$ \\
\hline C62 & C57 & $\mathrm{C} 58$ & C59 & $0.3(2)$ & C57 & $\mathrm{C} 58$ & $\mathrm{C} 59$ & C60 & $-1.7(3)$ \\
\hline C58 & C59 & $\mathrm{C} 60$ & C61 & $1.5(3)$ & C59 & $\mathrm{C} 60$ & C61 & C62 & $0.2(3)$ \\
\hline C60 & C61 & C62 & $\mathrm{C} 57$ & $-1.7(3)$ & $\mathrm{Si} 4$ & C63 & C64 & C65 & $-174.15(11)$ \\
\hline $\mathrm{Si} 4$ & C63 & C68 & C67 & $174.38(11)$ & C64 & C63 & C68 & C67 & $-2.4(3)$ \\
\hline C68 & C63 & C64 & C65 & $2.7(3)$ & C63 & C64 & C65 & C66 & $-0.4(3)$ \\
\hline 64 & C65 & C66 & C67 & $-2.2(3)$ & C65 & C66 & C67 & C68 & $2.5(3)$ \\
\hline C66 & C67 & C68 & C63 & $-0.1(3)$ & $\mathrm{P} 2$ & C69 & $\mathrm{C} 70$ & $\mathrm{C} 71$ & $177.11(12)$ \\
\hline $\mathrm{P} 2$ & C69 & $\mathrm{C} 74$ & $\mathrm{C} 73$ & $-178.93(12)$ & $\mathrm{C} 70$ & C69 & $\mathrm{C} 74$ & $\mathrm{C} 73$ & $-1.6(3)$ \\
\hline C74 & C69 & $\mathrm{C} 70$ & C71 & $-0.4(3)$ & C69 & $\mathrm{C} 70$ & $\mathrm{C} 71$ & $\mathrm{C} 72$ & $1.3(3)$ \\
\hline $\mathrm{C} 70$ & C71 & $\mathrm{C} 72$ & $\mathrm{C} 73$ & $-0.2(3)$ & C71 & $\mathrm{C} 72$ & $\mathrm{C} 73$ & $\mathrm{C} 74$ & $-1.8(3)$ \\
\hline $\mathrm{C} 72$ & $\mathrm{C} 73$ & $\mathrm{C} 74$ & C69 & $2.7(3)$ & P3 & $\mathrm{C} 75$ & $\mathrm{C} 76$ & $\mathrm{C} 77$ & $178.58(13)$ \\
\hline P3 & $\mathrm{C} 75$ & $\mathrm{C} 80$ & C79 & $-178.74(12)$ & $\mathrm{C} 76$ & $\mathrm{C} 75$ & $\mathrm{C} 80$ & C79 & $1.3(3)$ \\
\hline $\mathrm{C} 80$ & $\mathrm{C} 75$ & $\mathrm{C} 76$ & $\mathrm{C} 77$ & $-1.4(3)$ & $\mathrm{C} 75$ & $\mathrm{C} 76$ & $\mathrm{C} 77$ & $\mathrm{C} 78$ & $0.5(3)$ \\
\hline $\mathrm{C} 76$ & $\mathrm{C} 77$ & $\mathrm{C} 78$ & C79 & $0.7(4)$ & $\mathrm{C} 77$ & $\mathrm{C} 78$ & C79 & $\mathrm{C} 80$ & $-0.8(3)$ \\
\hline $\mathrm{C} 78$ & C79 & $\mathrm{C} 80$ & $\mathrm{C} 75$ & $-0.2(3)$ & $\mathrm{P} 3$ & $\mathrm{C} 81$ & $\mathrm{C} 82$ & $\mathrm{C} 83$ & $-176.94(12)$ \\
\hline P3 & $\mathrm{C} 81$ & $\mathrm{C} 86$ & $\mathrm{C} 85$ & $177.76(12)$ & $\mathrm{C} 82$ & $\mathrm{C} 81$ & $\mathrm{C} 86$ & $\mathrm{C} 85$ & $-1.9(3)$ \\
\hline C86 & $\mathrm{C} 81$ & $\mathrm{C} 82$ & $\mathrm{C} 83$ & $2.8(3)$ & $\mathrm{C} 81$ & $\mathrm{C} 82$ & $\mathrm{C} 83$ & $\mathrm{C} 84$ & $-1.6(3)$ \\
\hline $\mathrm{C} 82$ & $\mathrm{C} 83$ & $\mathrm{C} 84$ & $\mathrm{C} 85$ & $-0.3(3)$ & $\mathrm{C} 83$ & $\mathrm{C} 84$ & $\mathrm{C} 85$ & $\mathrm{C} 86$ & $1.2(3)$ \\
\hline C84 & $\mathrm{C} 85$ & $\mathrm{C} 86$ & C81 & $-0.0(3)$ & P3 & $\mathrm{C} 87$ & $\mathrm{C} 88$ & C89 & $-172.80(11)$ \\
\hline P3 & $\mathrm{C} 87$ & C92 & C91 & $173.69(11)$ & C88 & $\mathrm{C} 87$ & C92 & C91 & $0.7(3)$ \\
\hline C92 & $\mathrm{C} 87$ & $\mathrm{C} 88$ & C89 & $0.0(3)$ & $\mathrm{C} 87$ & $\mathrm{C} 88$ & $\mathrm{C} 89$ & $\mathrm{C} 90$ & $-0.5(3)$ \\
\hline C88 & C89 & C90 & C91 & $0.3(3)$ & C89 & $\mathrm{C} 90$ & C91 & C92 & $0.4(3)$ \\
\hline C90 & C91 & C92 & $\mathrm{C} 87$ & $-0.9(3)$ & N1 & C93 & C94 & $\mathrm{C} 5$ & $-109.1(9)$ \\
\hline N1 & C93 & $\mathrm{C} 95$ & $\mathrm{C} 2$ & $-57.2(10)$ & N1 & C93 & C95 & $\mathrm{C} 4$ & $-63.2(10)$ \\
\hline N1 & C93 & C95 & $\mathrm{C} 5$ & $111.8(17)$ & $\mathrm{C} 2$ & C93 & C95 & $\mathrm{C} 2$ & $-0.0(2)$ \\
\hline $\mathrm{C} 2$ & C93 & $\mathrm{C} 95$ & $\mathrm{C} 4$ & $-6.0(8)$ & $\mathrm{C} 2$ & C93 & C95 & $\mathrm{C} 5$ & $169(2)$ \\
\hline $\mathrm{C} 3$ & C93 & C94 & $\mathrm{C} 5$ & 133.7(9) & $\mathrm{C} 3$ & C93 & C95 & $\mathrm{C} 2$ & $59.8(13)$ \\
\hline $\mathrm{C} 3$ & C93 & $\mathrm{C} 95$ & $\mathrm{C} 4$ & $53.8(14)$ & $\mathrm{C} 3$ & $\mathrm{C} 93$ & C95 & $\mathrm{C} 5$ & $-131.2(16)$ \\
\hline C5 & C93 & C94 & $\mathrm{C} 5$ & $0.00(16)$ & $\mathrm{C} 5$ & C93 & C95 & $\mathrm{C} 2$ & $-169(2)$ \\
\hline $\mathrm{C} 5$ & C93 & C95 & $\mathrm{C} 4$ & $-175(2)$ & $\mathrm{C} 5$ & C93 & C95 & $\mathrm{C} 5$ & $-0.0(3)$ \\
\hline C94 & C93 & C95 & $\mathrm{C} 2$ & $179.2(15)$ & C94 & C93 & C95 & $\mathrm{C} 4$ & 173.2(9) \\
\hline C94 & C93 & $\mathrm{C} 95$ & $\mathrm{C} 5$ & $-12(2)$ & C95 & C93 & C94 & $\mathrm{C} 5$ & $6.2(11)$ \\
\hline
\end{tabular}




\section{References}

(1) Suginome, M.; Oike, H.; Park, S. -S.; Ito, Y. Bull. Chem. Soc. Jpn., 1996, 69, 289-299.

(2) Tacke, R.; Heemann, J.; Penka, M.; Richter, I.; Wagner, B. Z. Naturforsch., 2002, 57b, 731-735.

(3) Frisch, M. J.; Trucks, G. W.; Schlegel, H. B.; Scuseria, G. E.; Robb, M. A.; Cheeseman, J. R.; Scalmani, G.; Barone, V.; Mennucci, B.; Petersson, G. A.; Nakatsuji, H.; Caricato, M.; Li, X.; Hratchian, H. P.; Izmaylov, A. F.; Bloino, J.; Zheng, G.; Sonnenberg, J. L.; Hada, M.; Ehara, M.; Toyota, K.; Fukuda, R.; Hasegawa, J.; Ishida, M.; Nakajima, T.; Honda, Y.; Kitao, O.; Nakai, H.; Vreven, T.; Montgomery, J. A. Jr.; Peralta, J. E.; Ogliaro, F.; Bearpark, M.; Heyd, J. J.; Brothers, E.; Kudin, K. N.; Staroverov, V. N.; Keith, T.; Kobayashi, R.; Normand, J.; Raghavachari, K;. Rendell, A.; Burant, J. C.; Iyengar, S. S.; Tomasi, J.; Cossi, M.; Rega, N.; Millam, J. M.; Klene, M.; Knox, J. E.; Cross, J. B.; Bakken, V.; Adamo, C.; Jaramillo, J;. Gomperts, R.; Stratmann, R. E.; Yazyev, O.; Austin, A. J.; Cammi, R.; Pomelli, C.; Ochterski, J. W.; Martin, R. L.; Morokuma, K.; Zakrzewski, V. G.; Voth, G. A.; Salvador, P.; Dannenberg, J. J.; Dapprich, S.; Daniels, A. D.; Farkas, O.; Foresman, J. B.; Ortiz, J. V.; Cioslowski, J.; Fox, D. J. Gaussian 09, Revision C.01; Gaussian, Inc., Wallingford, CT, 2010.

(4) Perdew, J.; Ernzerhof M.; Burke, K. J. Chem. Phys,. 1996, 105, 9982-9985; (b) Adamo C.; Barone, V. J. Chem. Phys., 1999, 110, 6158-6170.

(5) Zhao, Y.; Truhlar, D. G. Theor. Chem. Acc., 2008, 120, 215-241.

(6) (a) Becke, A. D. Phys. Rev. A, 1988, 38, 3098-3100; (b) Becke, A. D. J. Chem. Phys., 1993, 98, 1372-1377; (c) Becke, A. D. J. Chem. Phys., 1993, 98, 5648-5652; (d) Perdew, J. P.; Chevary, J. A.; Vosko, S. H.; Jackson, K. A.; Pederson, M. R.; Singh, D. J.; Fiolhais, C. Phys. Rev. B, 1992, 46, 6671-6687; (e) Perdew,; J. P. Wang, Y. Phys. Rev. B, 1992, 45, 13244-13249; (f) Perdew, J. P.; Chevary, J. A.; Vosko, S. H.; Jackson, K. A.; Pederson, M. R.; Singh, D. J.; Fiolhais, C. Phys. Rev. B, 1993, 48, 4978; (g) Perdew, J. P.; Burke, K.; Wang, Y. Phys. Rev. B, 1996, 54, 16533-16539.

(7) (a) Becke, A. D. Phys. Rev. A, 1988, 38, 3098-3100; (b) Lee, C.; Yang, W.; Parr, R. G. Phys. Rev. B, 1988, 37, 785-789; (c) Becke, A. D. J. Chem. Phys., 1993, 98, 5648-5652.

(8) Andrae, D.; Häußermann, U.; Dolg, M.; Stoll, H.; Preuß, H. Theor. Chim. Acta, 1990, 77, 123-14.

(9) (a) Gordon, M. S. Chem. Phys. Lett., 1980, 76, 163-168; (b) Hariharan, P. C.; Pople, J. A. Mol. Phys., 1974, 27, 209-214; (c) Hariharan, P. C.; Pople, J. A. Theor. Chem. Acc., 1973, 28, 213-222; (d) Hehre, W. J.; Ditchfield, R.; Pople, J. A. J. Chem. Phys., 1972, 56, 2257-2261; (e) Ditchfield, R.; Hehre, W. J.; Pople, J. A. J. Chem. Phys., 1971, 54, 724-728.

(10) SIR2008: Burla, M. C.; Caliandro, R.; Camalli, M.; Carrozzini, B.; Cascarano, G. L.; De Caro, L.; Giacovazzo, C.; Polidori, G.; Siliqi, D.; Spagna, R. J. Appl. Cryst., 2007, 40, 609-613.

(11) International Tables for Crystallography, Vol. C; (Ed. Wilson, A. J. C.) Kluwer Academic Publishers, Dordrecht, Netherlands, Table 6.1.1.4, 1992, pp. 572.

(12) Ibers, J. A.; Hamilton, W. C. Acta Cryst., 1964, 17, 781-782.

(13) Creagh, D. C.; McAuley, W. J. in International Tables for Crystallography, Vol C; (Ed. Wilson, A. J. C.), Kluwer Academic Publishers, Dordrecht, Netherlands, Table 4.2.6.8, 1992, pages 219-222.

(14) Creagh, D. C.; Hubbell, J. H. in International Tables for Crystallography, Vol C; (Ed. Wilson, A. J. 
C.), Kluwer Academic Publishers, Boston, Table 4.2.4.3, 1992, pages 200-206.

(15) CrystalStructure 4.2.5: Crystal Structure Analysis Package, Rigaku Corporation (2000-2017). Tokyo 196-8666, Japan.

(16) SHELXL Version 2017/1: Sheldrick, G. M. Acta Cryst., 2008, A64, 112-122. 